\title{
Meta-Invariant Operators over Cayley-Dickson Algebras and Spectra
}

\author{
S. V. Ludkovsky \\ Department of Applied Mathematics, Moscow State Technical University, \\ Moscow, Russia \\ Email: Ludkowski@mirea.ru
}

Received June 28, 2012; revised September 2, 2012; accepted September 17, 2012

\begin{abstract}
A class of meta-invariant operators over Cayley-Dickson algebra is studied. Their spectral theory is investigated. Moreover, theorems about spectra of generalized unitary operators and their semigroups are demonstrated.
\end{abstract}

Keywords: Hypercomplex Numbers; Cayley-Dickson Algebras; Operator; Operator Algebra; Spectra; Spectral Measure

\section{Introduction}

The Cayley-Dickson algebras are algebras over the real field $\boldsymbol{R}$, but they are not algebras over the complex field $\boldsymbol{C}$, since each embedding of $\boldsymbol{C}$ into $\mathcal{A}_{r}$ with $r \geq 2$ is not central. That is why the theory of algebras of operators over the Cayley-Dickson algebras $\mathcal{A}_{r}$ with $r \geq 2$ is different from algebras of operators over the complex field $\boldsymbol{C}$. On the other hand, such theory has many specific features in comparison with the general theory of algebras of operators over $\boldsymbol{R}$ due to the graded structures of algebras $\mathcal{A}_{r}$. Moreover, the Cayley-Dickson algebra $\mathcal{A}_{r}$ with $r \geq 3$ can not be realized as the subalgebra of any algebra of matrices over $\boldsymbol{R}$, since these algebras $\mathcal{A}_{r}$ with $r \geq 3$ are not associative, but the matrix algebra is associative.

The results of this paper can be used also for the development of non-commutative geometry, super-analysis, quantum mechanics over $\mathcal{A}_{r}$, and the theory of representations of topological and Lie groups and supergroups which may be non locally compact, for example, of the type of the group of diffeomorphisms and the group of loops or wraps of manifolds over $\mathcal{A}_{r}$ (see [1-8]).

As examples of unbounded operators over $\mathcal{A}_{r}$ serve differential operators, including operators in partial derivatives. For example, the Klein-Gordon-Fock or Dirac operators are used in the theory of spin manifolds [9], but each spin manifold can be embedded in the quaternion manifold [10].

The skew field of quaternions $\boldsymbol{H}$ has the automorphism $\eta$ of order two $\eta: z \mapsto \tilde{z}$, where

$$
\tilde{z}=w_{1}-w_{i} i-w_{j} j-w_{k} k,
$$

$$
z=w_{1}+w_{i} i+w_{j} j+w_{k} k ; w_{1}, \cdots, w_{k} \in \boldsymbol{R} .
$$

There is the norm in $\boldsymbol{H}$ such that $|z|=|z \tilde{z}|^{1 / 2}$, consequently, $\tilde{z}=|z|^{2} z^{-1}$.

The algebra $\boldsymbol{K}$ of octonions (octave, the Cayley algebra) is defined as the eight dimensional algebra over $\boldsymbol{R}$ with a basis, for example,

1) $b_{3}:=b:=\{1, i, j, k, l, i l, j l, k l\}$ such that;

2) $i^{2}=j^{2}=k^{2}=l^{2}=-1, \quad j=k, \quad j i=-k, j k=i$, $k j=-i, \quad k i=j, i k=-j, \quad l i=-i l, \quad j l=-l j, k l=-l k$;

3) $(\alpha+\beta l)(\gamma+\delta l)=(\alpha \gamma-\tilde{\delta} \beta)+(\delta \alpha+\beta \tilde{\gamma}) l$ it is the law of multiplication in $\boldsymbol{K}$ for each $\alpha, \beta, \gamma, \delta \in \boldsymbol{H}, \quad \xi:=\alpha+\beta l \in \boldsymbol{K}, \quad \eta:=\gamma+\delta l \in \boldsymbol{K}$, $\tilde{z}:=v-w i-x j-y k$ for quaternions $z=v+w i+x j+y k \in \boldsymbol{H}$ with $v, w, x, y \in \boldsymbol{R}$.

The algebra of octonions is neither commutative, nor associative, since $(i j) l=k l, i(j l)=-k l$, but it is distributive and the real field $\boldsymbol{R} 1$ is its center. If $\xi:=\alpha+\beta l \in \boldsymbol{K}$, then

4) $\tilde{\xi}:=\tilde{\alpha}-\beta l$ is called the adjoint element for $\xi$, where $\alpha, \beta \in \boldsymbol{H}$. In addition we have the identities:

5) $(\xi \eta)^{\tilde{*}}=\tilde{\eta} \tilde{\xi}, \tilde{\xi}+\tilde{\eta}=(\xi+\eta)^{\tilde{\gamma}}$ and $\xi \tilde{\xi}=|\alpha|^{2}+|\beta|^{2}$, where $|\alpha|^{2}=\alpha \tilde{\alpha}$, so that

6) $\xi \tilde{\xi}=:|\xi|^{2}$ and $|\xi|$ is the norm in $\boldsymbol{K}$. This implies that

7) $|\xi \eta|=|\xi||\eta|$,

consequently, $\boldsymbol{K}$ does not contain the divisors of zero (see also [11]). The multiplication of octonions satisfies the equations:

8) $(\xi \eta) \eta=\xi(\eta \eta)$

9) $\xi(\xi \eta)=(\xi \xi) \eta$, 
which define the property of alternativity of algebras. In particular, $(\xi \xi) \xi=\xi(\xi \xi)$.

Preliminary results on operator theory and operator algebras over quaternions and octonions were published in [12]. This articles develops further operator theory already over general Cayley-Dickson algebras.

In the third section of this article the theory of unbounded operators is described, as well as bounded quasilinear operators in the Hilbert spaces $\boldsymbol{X}$ over the CayleyDickson algebras $\mathcal{A}_{r}$. At the same time the analog of the scalar product in $\boldsymbol{X}$ is defined with values in the CayleyDickson algebra $\mathcal{A}_{r}$. For the spectral theory of such operators there are defined and used also graded operators of projections and graded projection valued measures. The linearity of operators over the algebra $\mathcal{A}_{r}$ with $r \geq 2$ is already not worthwhile because of the non-commutativity of $\mathcal{A}_{r}$, therefore there is introduced the notion of quasi-linear operators.

At the same time graded projection valued measures in the general case may be non-commutative and non-associative. Because of the non-commutativity of the Cayley-Dickson algebras $\mathcal{A}_{r}$ with $r \geq 2$ commutative algebras $\mathcal{Z}$ over them withdraw their importance, since they can be only trivial $\mathcal{Z} \mathcal{A}_{r}=\mathcal{Z}=\{0\}$, moreover, over the Cayley-Dickson algebra $\mathcal{A}_{r}$ with $r \geq 3$ the associativity may lose its importance. Therefore, in the third section the notion of quasi-commutative algebra metainvariant over $\mathcal{A}_{r}$ is introduced. Principles of the theory of bounded and unbounded operators are given there. Spectral theory of self-adjoint and generalized unitary operators is exposed. In the third section spectral theory of normal operators is described. For algebras of normal meta-invariant over $\mathcal{A}_{r}$ operators the theorem generalizing that of Gelfand-Mazur is proved. An analog of Stone's theorem over $\mathcal{A}_{r}$ for the one parameter families of unitary operators is demonstrated.

Definitions and notations of the preceding work [12] are used below.

\section{Preliminaries}

1) Notations and Definitions. Let $\mathcal{A}_{r}$ denote the real Cayley-Dickson algebra with generators $i_{0}, \cdots, i_{2^{r}-1}$ such that $i_{0}=1, \quad i_{j}^{2}=-1$ for each $j \geq 1, \quad i_{j} i_{k}=-i_{k} i_{j}$ for each $j \neq k \geq 1$.

The Cayley-Dickson algebra $\mathcal{A}_{r+1}$ is formed from the algebra $\mathcal{A}_{r}$ with the help of the doubling procedure by generator $i_{2^{r}}$, in particular, $\mathcal{A}_{0}:=\boldsymbol{R}$ is the real field, $\mathcal{A}_{1}=\boldsymbol{C}$ coincides with the field of complex numbers, $\mathcal{A}_{2}=\boldsymbol{H}$ is the skew field of quaternions, $\mathcal{A}_{3}$ is the algebra of octonions, $\mathcal{A}_{4}$ is the algebra of sedenions. The algebra $\mathcal{A}_{r}$ is power associative, that is,

1) $z^{n+m}=z^{n} z^{m}$ for each $n, m \in N$ and $z \in \mathcal{A}_{r}$.

It is non-associative and non-alternative for each $r \geq 4$.

The Cayley-Dickson algebras are-algebras, that is, there is a real-linear mapping $\mathcal{A}_{r} \ni a \mapsto a^{*} \in \mathcal{A}_{r}$ such that

2) $a^{* *}=a$,

3) $(a b)^{*}=b^{*} a^{*}$ for each $a, b \in \mathcal{A}_{r}$. Then they are nicely normed, that is,

4) $a+a^{*}=: 2 \operatorname{Re}(a) \in R$ and

5) $a a^{*}=a^{*} a>0$ for each $0 \neq a \in \mathcal{A}_{r}$. The norm in it is defined by the equation:

6) $|a|^{2}:=a a^{*}$.

We also denote $a^{*}$ by $\tilde{a}$. Each non-zero CayleyDickson number $0 \neq a \in \mathcal{A}_{r}$ has the multiplicative inverse given by $a^{-1}=a^{*} /|a|^{2}$.

The doubling procedure is as follows. Each $z \in \mathcal{A}_{r+1}$ is written in the form $z=a+b \mathbf{1}$, where $\mathbf{1}^{2}=-1, \mathbf{1} \notin \mathcal{A}_{r}$, $a, b \in \mathcal{A}_{r}$. The addition is componentwise. The conjugate of a Cayley-Dickson number $z$ is prescribed by the formula:

7) $z^{*}:=a^{*}-b \mathbf{1}$. The multiplication is given by Equation

8) $(\alpha+\beta \mathbf{1})(\gamma+\delta \mathbf{1})=(\alpha \gamma-\tilde{\delta} \beta)+(\delta \alpha+\beta \tilde{\gamma}) \mathbf{1}$.

for each $\alpha, \beta, \gamma, \delta \in \mathcal{A}_{r}, \quad \xi:=\alpha+\beta \mathbf{1} \in \mathcal{A}_{r+1}$, $\eta:=\gamma+\delta \mathbf{1} \in \mathcal{A}_{r+1}$.

The basis of $\mathcal{A}_{r+1}$ over $\boldsymbol{R}$ is denoted by $\boldsymbol{b}_{r+1}:=\boldsymbol{b}:=\left\{1, i_{1}, \cdots, i_{2^{r+1}-1}\right\}$, where $i_{s}^{2}=-1$ for each $1 \leq s \leq 2^{r+1}-1, \quad i_{2^{r}}:=\mathbf{1}$ is the additional element of the doubling procedure of $\mathcal{A}_{r+1}$ from $\mathcal{A}_{r}$, choose

$i_{2^{r}+m}=i_{m} 1$ for each $m=1, \cdots, 2^{r}-1, i_{0}:=1$.

Put by the definition $\operatorname{Im}(z)=z-\operatorname{Re}(z)$ to be the imaginary part of a Cayley-Dickson number $z$.

It is possible to consider the Cayley-Dickson algebras of greater real dimensions.

2) Definition. Let $\mathcal{A}_{\infty}$ denote the family consisting of elements $z=\sum_{s \in b} w_{s} s$ such that $\tilde{z}:=w_{1}-\sum_{s \in \hat{b}} w_{s} s$,

$$
z \tilde{z}=:|z|^{2}=\sum_{s \in b} w_{s}^{2}<\infty,
$$

where

$$
\boldsymbol{b}:=\boldsymbol{b}_{\infty}:=\bigcup_{r=2}^{\infty} \boldsymbol{b}_{r}=\left\{1, i_{1}, i_{2}, \cdots, i_{2^{r}}, \cdots\right\},
$$

$\hat{b}:=b \backslash\{1\}, w_{s} \in \boldsymbol{R}$ for each $s$.

3) Theorem. The family $\mathcal{A}_{\infty}$ has the structure of the normed power-associative left and right distributive algebra over the real field $\boldsymbol{R}$ with the external involution of order two.

Proof. Let $\mathcal{I}_{\infty}:=\left\{z \in \mathcal{A}_{\infty}: \operatorname{Re}(z)=0\right\}$. Then each element $M \in \mathcal{I}_{\infty}$ is the limit of the sequence $M_{r} \in \mathcal{I}_{r}$, also $|z|=: \rho$ is the limit of the sequence $\rho_{r}:=\left|z_{r}\right|$, where $z_{r} \in \mathcal{A}_{r}$. Therefore, there exists the limit 


$$
\begin{aligned}
Z & =\lim _{r \rightarrow \infty} Z_{r}=\lim _{r \rightarrow \infty} \rho_{r}\left\{\cos \left|M_{r}\right|+\left[\left(\sin \left|M_{r}\right|\right) /\left|M_{r}\right|\right] M_{r}\right\} \\
& =\rho\{\cos |M|+[(\sin |M|) /|M|] M\}=\rho \exp (M) .
\end{aligned}
$$

Using the polar coordinates $(\rho, M)$ prove the powerassociativity. There exists the natural projection $P_{r}$ from $\mathcal{A}_{\infty}$ onto $\mathcal{A}_{r}$ for each $r \geq 2$ given by the formulas:

$$
M_{r}:=\left\{\sum_{s \in \hat{b}_{r}} m_{s} s\right\}|M|\left[\sum_{s \in \hat{b}_{r}} m_{s}^{2}\right]^{-1 / 2} \text { for } \sum_{s \in \hat{b}_{r}} m_{s}^{2} \neq 0
$$

and $M_{r}=0$ in the contrary case for each

$$
M=\sum_{s \in \hat{b}} m_{s} s \in \mathcal{I}_{\infty},
$$

where $m_{s} \in \boldsymbol{R}$ for each $s \in \hat{b}$; then

$$
z_{r}:=P_{r}(z)=\rho_{r}\left\{\cos \left|M_{r}\right|+\left[\left(\sin \left|M_{r}\right|\right) /\left|M_{r}\right|\right] M_{r}\right\}
$$

where

$$
\rho_{r}=\left(\sum_{s \in b_{r}} w_{s}^{2}\right)^{1 / 2}, z=\sum_{s \in \boldsymbol{b}} w_{s} s, \lim _{0 \neq \phi \rightarrow 0} \sin (\phi) / \phi=1 .
$$

Then the limit exists $\lim _{r \rightarrow \infty} z_{r}=z$ relative to the norm $|z|$ in $\mathcal{A}_{\infty}$. Therefore, for each $n \in \boldsymbol{Z}$ there exists the limit

$$
\lim _{r \rightarrow \infty}\left(\rho_{r}\right)^{n} \exp \left(n M_{r}\right)=\rho^{n} \exp (n M)=z^{n},
$$

consequently, $\mathcal{A}_{\infty}$ is power-associative, since each $\mathcal{A}_{r}$ is power-associative, cos and sin are continuous functions. Evidently, $\mathcal{A}_{\infty}$ is the $\boldsymbol{R}$-linear space.

The continuity of multiplication relative to the norm $|z|$ follows from the inequalities

$$
\begin{aligned}
\left|\xi_{r} \eta_{r}-\psi_{r} \zeta_{r}\right| & \leq\left|\xi_{r} \eta_{r}-\xi_{r} \zeta_{r}\right|+\left|\xi_{r} \zeta_{r}-\psi_{r} \zeta_{r}\right| \\
& \leq|\xi||\eta-\zeta|+|\xi-\psi||\zeta|
\end{aligned}
$$

and taking the limit with $r$ tending to the infinity, since $\left|\xi_{r}\right| \leq|\xi|$ and $\left|\xi_{r} \eta_{r}\right| \leq\left|\xi_{r}\right|\left|\eta_{r}\right|$ for each $\xi_{r}, \eta_{r} \in \mathcal{A}_{r}$ and for each $r \in N$. The left and right distributiveness

$$
(\xi+\psi) \zeta=\xi \zeta+\psi \zeta \text { and } \zeta(\xi+\psi)=\zeta \xi+\zeta \psi
$$

follow from taking the limit with $r$ tending to the infinity and such distributivity in each $\mathcal{A}_{r}$.

The involution $z \mapsto \tilde{z}=: z^{*}$ in $\mathcal{A}_{r}$ is of order two, since $\left(z^{*}\right)^{*}=z$. It is external, since there is not any finite algebraic relation with constants in $\mathcal{A}_{\infty}$ transforming the variable $z \in \mathcal{A}_{\infty}$ into $z^{*}$. The relation

$$
\begin{aligned}
Z^{*} & =\lim _{r \rightarrow \infty}\left(2^{r}-2\right)^{-1}\left\{-z_{r}+\sum_{s \in \hat{b}_{r}} s\left(z_{r} s^{*}\right)\right\} \\
& =\lim _{r \rightarrow \infty}\left(2^{r}-2\right)^{-1}\left\{-z+\sum_{s \in \hat{b}_{r}} s\left(z s^{*}\right)\right\}
\end{aligned}
$$

is of infinite order. The latter limit exists point-wise, but does not converge uniformly in any ball of positive radius relative to the norm in the algebra $\mathcal{A}_{\infty}$. The relations of the type $z_{r}^{*}=l_{r+1}\left(z_{r} l_{r+1}^{*}\right)$ in $\mathcal{A}_{r}$ use external automorphism with $l_{r+1}:=i_{2^{r}} \in \mathcal{A}_{r+1} \backslash \mathcal{A}_{r}$, moreover, the latter relation is untrue for $z^{*}$ and $z \in \mathcal{A}_{\infty}$ instead of $z_{r} \in \mathcal{A}_{r}$

No any finite set of non-zero constants $a_{1}, \cdots, a_{n} \in \mathcal{A}_{\infty}$ can provide the automorphism $z \mapsto \tilde{z}$ of $\mathcal{A}_{\infty}$. To prove this consider an $\boldsymbol{R}$-subalgebra $\Upsilon_{M_{1}, \cdots, M_{n}}$ of $\mathcal{A}_{\infty}$ generated by $\left\{M_{1}, \cdots, M_{n}\right\}$, where $a_{j}=\left|a_{j}\right| \mathrm{e}^{M_{j}}$, $M_{j} \in \mathcal{I}_{\infty}$. Since $a_{1} a_{1}^{*}=\left|a_{1}\right|^{2}>0$, then $R\left|a_{1}\right|^{2}=R \subset \Upsilon_{M_{1}, \cdots, M_{n}}$, hence $1 \in \Upsilon_{M_{1}, \cdots, M_{n}}$. If $\Upsilon_{M_{1}, \cdots, M_{n}}=\boldsymbol{R}$, then it certainly can not provide the automorphism $Z \mapsto \tilde{z}$ of $\mathcal{A}_{\infty}$. Consider $\Upsilon_{M_{1}, \cdots, M_{n}} \neq \boldsymbol{R}$, without loss of generality suppose $a_{1} \notin \boldsymbol{R}$. There is the scalar product $\operatorname{Re}(z \tilde{y})$ in $\mathcal{A}_{\infty}$ for each $z, y \in \mathcal{A}_{\infty}$. Let $b_{1}$ be the projection of $a_{1}$ in a subspace of $\mathcal{A}_{\infty}$ orthogonal to $\boldsymbol{R} 1$, then by our supposition $b_{1} \neq 0$ and $b_{1} \in \mathcal{I}_{\infty}$. Therefore, we get $b_{1}^{2} /\left|b_{1}\right|^{2}=-1$, consequently, $\Upsilon_{M_{1}}$ is isomorphic to $C$. Certainly, no any proper Cayley-Dickson subalgebra $\mathcal{A}_{r}, r \in N$, can provide the automorphism $Z \mapsto \tilde{Z}$ of $\mathcal{A}_{\infty}$.

Therefore, without loss of generality suppose, that $\Upsilon_{M_{1}, \cdots, M_{n}}$ is not isomorphic to $\boldsymbol{C}$ and $a_{2} \notin \boldsymbol{C}$. If $M, N \in \mathcal{I}_{r}$ and $\operatorname{Re}\left(M N^{*}\right)=0$, then $M N \in \mathcal{I}_{r}$ and hence $(M N)^{*}=N M=-M N$, since $A^{*}=-A$ for each $A \in \mathcal{I}_{r}$. Let $b_{2}$ be the projection of $M_{2}$ in a subspace of $\mathcal{A}_{\infty}$ orthogonal to $\Upsilon_{M_{1}}$ relative to the scalar product $\operatorname{Re}(z \tilde{y})$. Then $b_{2} \neq 0$ by our supposition and $b_{2} \in \mathcal{I}_{\infty}$, $b_{2}^{2} /\left|b_{2}\right|^{2^{2}}=-1$, hence after the doubling procedure with $b_{2} /\left|b_{2}\right|$ we get, that $\Upsilon_{M_{1}, M_{2}}$ is a subalgebra of $\mathcal{A}_{4}$.

Then proceed by induction. Suppose that $\Upsilon_{M_{1}, \cdots, M_{k}}$ is a subalgebra of $\mathcal{A}_{2^{k}}, k \in N, k<n$. Since $\mathcal{A}_{2^{k}}$ can not provide the automorphism $z \mapsto \tilde{z}$ of $\mathcal{A}_{\infty}$, suppose without loss of generality, that $a_{k+1} \notin \Upsilon_{M_{1}, \cdots, M_{k}}$ and consider the orthogonal projection $b_{k+1}$ of $M_{k+1}$ in a subspace of $\mathcal{A}_{\infty}$ orthogonal to $\Upsilon_{M_{1}, \cdots, M_{k}}$ relative to the scalar product $\operatorname{Re}(z \tilde{y})$. Then $b_{k+1} \neq 0$ and $b_{k+1} \in \mathcal{I}_{\infty}$, $b_{k+1}^{2} /\left|b_{k+1}\right|^{2}=-1$. Therefore, the doubling procedure with $b_{k+1} /\left|b_{k+1}\right|$ gives the algebra $\Upsilon_{M_{1}, \cdots, M_{k+1}}$ which is the subalgebra of $\mathcal{A}_{2^{k}+1}$, etc. As the result $\Upsilon_{M_{1}, \cdots, M_{n}}$ is the subalgebra of $\mathcal{A}_{2^{n}}$ and can not provide the automorphism $\mathrm{z} \mapsto \tilde{z}$ of $\mathcal{A}_{\infty}$, where $a_{1}, \cdots, a_{n} \in \Upsilon_{M_{1}, \cdots, M_{n}}$ due to the formula of the polar decomposition of Cayley-Dickson numbers $a=|a| \mathrm{e}^{t M}$ with a real parameter $t=t(a) \in \boldsymbol{R}$ and a purely imaginary parameter $M \in \mathcal{A}_{r}$ of the unit norm $|M|=1, \operatorname{Re}(M)=0$ (see also $\S 2.2$ [13]).

4) Note and Definition. Let $\Lambda$ denote a Hausdorff topological space with non-negative measure $\mu$ on a $\sigma$-algebra of all Borel subsets such that for each point $x \in \Lambda$ there exists an open neighborhood $U \ni x$ with 
$0<\mu(U)<\infty$. Consider a set of generators with real algebra $\left\{i_{x}: x \in \Lambda\right\}$ such that $i_{x} i_{y}=-i_{y} i_{x}$ for each $x \neq y \in \Lambda \backslash\{0\}$ and $i_{x}^{2}=-1$ for each $x \in \Lambda \backslash\{0\}$, where 0 is a marked point in $\Lambda$. Add to this set the unit $1=: i_{0}$ such that $b i_{x}=i_{x} b$ for each $b \in \boldsymbol{R}$ and $x \in \Lambda$. We consider also finite products of these generators in accordance with $\S 1$, where each $i_{x}$ with $x \in \Lambda \backslash\{0\}$ is considered as the doubling generator.

In the case of a finite set $\Lambda$ Cayley-Dickson algebra generated by such generators is isomorphic with $\mathcal{A}_{N-1}$, where $N=\operatorname{card}(\Lambda)$ is the cardinality of the set $\Lambda$.

For the infinite subset of generators $\left\{i_{0}, i_{x_{j}}: j \in N, x_{j} \in \Lambda\right\}$ the construction from $\S 2$ produces the algebra isomorphic with $\mathcal{A}_{\infty}$.

Therefore, we consider now the case $\operatorname{card}(\Lambda)>\aleph_{0}$. Due to the Kuratowski-Zorn lemma (see §I.4 in [14]) we can suppose, that $\Lambda$ is linearly ordered and this linear ordering gives intervals $(a, b):=\{x \in \Lambda: a<x<b\}$ being $\mu$-measurable, for example, $\Lambda=\boldsymbol{R}^{n} \times(\boldsymbol{R} / \mathbf{Z})^{m}$ has the natural linear ordering induced by the linear ordering from $\boldsymbol{R}$ and by the lexicographic ordering in the product, where $n, m \in \boldsymbol{N}$, or $m$ may be an ordinal of the cardinality $\operatorname{card}(m) \geq \aleph_{0}$.

Then consider a finite partition $\Lambda$ into a disjoint union $\Lambda=\bigcup_{j=0}^{p} \mathcal{A}_{j}$, where $x<y$ for each $x \in \mathcal{A}_{j}$ and $y \in \mathcal{A}_{1}$ for $j<l \leq p, \quad p \in N, 0 \in \mathcal{A}_{0}$. The family of such partitions we denote $Z$. Let $T \in Z, x_{j} \in \mathcal{A}_{j}$ be marked points. Then there exists a step function $f_{T}$ such that $f_{T}(x)=C_{j} i_{x_{j}}$ for each $x \in \mathcal{A}_{j}$, where $C_{j} \in \boldsymbol{R}$. Consider the norm

$$
\left\|f_{T}\right\|_{\Lambda}^{2}:=\int_{\Lambda} f_{T}(x) \tilde{f}_{T}(x) \mu(\mathrm{d} x),
$$

where

$$
\tilde{f}_{T}(x):=C_{0} \chi_{\mathcal{A}_{0}}(x) \delta_{0, x_{0}}-\sum_{x_{j} \neq 0} C_{j} \chi_{\mathcal{A}_{j}}(x) i_{x_{j}},
$$

$\chi_{\mathcal{A}}(x)=1$ for $x \in \mathcal{A}, \chi_{\mathcal{A}}(x)=0$ for $x \notin \mathcal{A}, \delta_{x, y}=1$ for $x=y, \delta_{x, y}=0$ while $x \neq y$. To each $f_{T}$ put the element

$$
z_{f_{T}}:=\sum_{j} C_{j} i_{x_{j}} \mu\left(\mathcal{A}_{j}\right) .
$$

The algebra which is the completion by the norm $\|*\|_{\Lambda}$ of the minimal algebra generated by the family of elements $z_{f_{T}}$ for $f_{T}$ from the family $\mathcal{F}$ of all step functions and all their ordered final products we denote by $\mathcal{A}_{\Lambda}$.

5) Theorem. The set $\mathcal{A}_{\Lambda}$ is the power-associative non-commutative non-associative algebra over $\boldsymbol{R}$ complete relative to the norm $\|*\|_{\Lambda}$ with the center $Z\left(\mathcal{A}_{\Lambda}\right)=\boldsymbol{R}$. Moreover, there are embeddings $\mathcal{A}_{\infty} \rightarrow \mathcal{A}_{\Lambda}$ for $\operatorname{card}(\Lambda) \geq \aleph_{0}$. The set of generators of the algebra
$\mathcal{A}_{\Lambda}$ has the cardinality card $(\Lambda)$ for $\operatorname{card}(\Lambda) \geq \operatorname{card}(N)$. There exists the function $\overrightarrow{\exp }\left(\int_{\Lambda} f(x) \mu(\mathrm{d} x)\right)$ of the ordered integral product from $\mathcal{A}_{\Lambda}$ onto $\mathcal{A}_{\Lambda}$.

Proof. For $\operatorname{card}(\Lambda) \leq \aleph_{0}$ the algebra $\mathcal{A}_{\Lambda}$ is isomorphic with $\mathcal{A}_{N-1}$ or $\mathcal{A}_{\infty}$ in accordance with $\S \S 1-4$. Thus it remains to consider the case $\operatorname{card}(\Lambda)>\aleph_{0}$.

For each $f_{T} \in \mathcal{F}$ it can be defined the ordered integral exponential product

$$
\begin{aligned}
& \overrightarrow{\exp }\left(\int_{\Lambda} f_{T}(x) \mu(\mathrm{d} x)\right) \\
& :=\left\{\exp \left(C_{0} \mu\left(\mathcal{A}_{0}\right) \pi i_{x_{0}} / 2\right) \cdots \exp \left(C_{p} \mu\left(A_{p}\right) \pi i_{x_{p}} / 2\right)\right\}_{q(p+1)}
\end{aligned}
$$

with $q(p+1)$ corresponding to the left order of brackets. Thus the embeddings of $\mathcal{A}_{\infty}$ into $\mathcal{A}_{\Lambda}$ exist. Then $Z\left(\mathcal{A}_{\Lambda}\right)=\boldsymbol{R}$.

The completion of the family $\mathcal{F}$ contains all functions of the type

$$
f(x)=\sum_{j} f_{j}(x) \chi_{\mathcal{A}_{j}}(x) i_{x_{j}},
$$

where $\left\{\mathcal{A}_{j}: j \in \boldsymbol{N}\right\}$ is the disjoint union of $\Lambda$, each $\mathcal{A}_{j}$ is $\mu$-measurable, $f_{j} \in L^{2}(\Lambda, \mu, \boldsymbol{R})$ and

$$
\lim _{n \rightarrow \infty} \sum_{j>n}\left\|f_{j}(x) \chi_{\mathcal{A}_{j}}(x)\right\|_{L^{2}(\Lambda, \mu, R)}^{2}=0 .
$$

Since $\exp (M)=\cos (|M|)+M \sin (|M|) /|M|$ for each $M \in \mathcal{I}_{\infty}$ with $\operatorname{Re}(M)=0$ and

$|\exp (M)-1| \leq \exp (|M|)-1$, for each $f \in \mathcal{A}_{\Lambda}$ there exists

$$
\lim _{F \ni f_{T} \rightarrow f} \overrightarrow{\exp }\left(\int_{\Lambda} f_{T}(x) \mu(\mathrm{d} x)\right)=: \overrightarrow{\exp }\left(\int_{\Lambda} f(x) \mu(\mathrm{d} x)\right)
$$

relative to $\|*\|_{\Lambda}$. From the identity $\exp \left(\pi i_{x} / 2\right)=i_{x}$ for each $x \in \Lambda \backslash\{0\}$ it follows, that the family of all elements of the type $\overrightarrow{\exp }\left(\int_{\Lambda} f(x) \mu(\mathrm{d} x)\right), f \in \mathcal{F}$ contains all generators of the embedded subalgebra $\mathcal{A}_{\infty}$ generated by the countable subfamily $\left\{i_{x_{j}}: j \in N\right\}$.

The completion $\tilde{\mathcal{F}}$ of the family $\mathcal{F}$ by the norm $\|*\|_{\Lambda}$ is the infinite dimensional linear subspace over $\boldsymbol{R}$ in $\mathcal{A}_{\Lambda}$.

All possible final ordered products from $\tilde{\mathcal{F}}$ and the completion of their $\boldsymbol{R}$-linear span by the norm $\|*\|_{\Lambda}$ produces $\mathcal{A}_{\Lambda}$. Then for each element from $\mathcal{A}_{\Lambda}$ there exists the representation in the form of the ordered integral exponential product. Since $\mathcal{A}_{\Lambda}$ is the algebra over $\boldsymbol{R}$ and $\operatorname{card}(\Lambda)^{\aleph_{0}}=\operatorname{card}(\Lambda)$, then the family of genera- 
tors of the algebra $\mathcal{A}_{\Lambda}$ has the cardinality $\operatorname{card}(\Lambda)$.

6) Weakened Topology. Suppose that $X$ is a real topological vector space. Let $X^{\prime}$ denote the vector space of all continuous $\boldsymbol{R}$ linear functionals on $X$. Each continuous $\boldsymbol{R}$ linear functional $g \in X^{\prime}$ defines a seminorm $p_{g}(x):=|g(x)|$. The topology generated by the semi-norms family $\left\{p_{g}: g \in X^{\prime}\right\}$ is called the weak or weakened topology for $X$ and it is usually denoted by $\tau_{w}(X)=\sigma\left(X, X^{\prime}\right)$.

7) Non-Commutative Riemann Sphere $\hat{\mathcal{A}}_{v}$. For $v<\infty$ the Cayley-Dickson algebra $\mathcal{A}_{v}$ is the locally compact topological space relative to the norm topology. In the finite dimensional over $\boldsymbol{R}$ case $v<\infty$, the weak and norm topologies on $\mathcal{A}_{v}$ are equivalent.

Henceforward, we denote $\mathcal{A}_{\Lambda}$ by $\mathcal{A}_{v}$ also, where the cardinality of $v$ is equal to the topological weight of the Cayley-Dickson algebra $\mathcal{A}_{\Lambda}$ relative to the norm topology, $\quad \operatorname{card}(v)=w\left(\mathcal{A}_{\Lambda}\right)$, where $\operatorname{card}(\Lambda) \geq \aleph_{0}$. Without loss of generality $v$ can be considered as an ordinal due to the Kuratowski-Zorn lemma. Generally embed $\mathcal{A}_{v}$ into the non-commutative unit sphere

$$
S_{2}(v):=\left\{(\xi, y) \in \boldsymbol{R} \oplus \mathcal{A}_{v}: \xi^{2}+\|y\|^{2}=1\right\}
$$

over the real field $\boldsymbol{R}$ such that $S_{2}(v) \backslash \theta\left(\mathcal{A}_{v}\right)=\{(1,0)\}$ is a singleton, where $\theta: \mathcal{A}_{v} \rightarrow S_{2}(v)$ denotes a continuous bijective mapping, $2 \leq v$ or may be $\operatorname{card}(v) \geq \aleph_{0}$. To realize an embedding one considers their union $S_{2}(v) \cup \mathcal{A}_{v}$ embedded into $\boldsymbol{R} \oplus \mathcal{A}_{v}$ such that they intersect by the set

$$
S_{2}(v) \cap \mathcal{A}_{v}=\left\{(0, y) \in \boldsymbol{R} \oplus \mathcal{A}_{v}:\|y\|=1\right\} .
$$

As a mapping one takes the stereographic projection $\theta^{-1}: S_{2}(v) \backslash\{(1,0)\} \rightarrow \mathcal{A}_{v}$ so that

$$
\theta^{-1}(\xi, y)=\frac{y}{1-\xi}=z \in \mathcal{A}_{v} .
$$

As the $\boldsymbol{R}$ linear normed space the Cayley-Dickson algebra $\mathcal{A}_{v}$ is isomorphic either with the Euclidean space $\boldsymbol{R}^{2^{v}}$ for $\operatorname{card}(v)<\aleph_{0}$ or with the real Hilbert space $l_{2}(v)$ of the topological weight $w\left(l_{2}(v)\right)=\operatorname{card}(v) \geq \aleph_{0}$. Consider on $l_{2}(v)$ the weak topology $\tau_{w}$ provided by the family of all continuous $\boldsymbol{R}$ linear functionals on $l_{2}(v)$. In view of the Alaoglu-Bourbaki Theorem (9.3.3) [15] the unit sphere $S_{2}(v)$ is compact relative to the weak topology $\tau_{w}$ on $S_{2}(v)$ inherited from the

$\boldsymbol{R} \oplus I_{2}(v)$ weak topology. Therefore, $\theta\left(\mathcal{A}_{v}\right)$ relative to the weak or weakened topology $\tau_{w}^{1}$ induced by the weak topology $\tau_{w}$ of $S_{2}(v)$ has the one-point compactification $\hat{\mathcal{A}}_{v}$ realized topologically as $S_{2}(v)$ (see also Theorem 3.5.11 about one-point Alexandroff compactification in [14]). That is, $\hat{\mathcal{A}}_{v} \backslash \mathcal{A}_{v}=\infty$.

The non-commutative analog $\hat{\mathcal{A}}_{v}$ of the Riemann sphere can be supplied with the topology inherited from the norm topology $\tau_{n}$ on $\boldsymbol{R} \oplus \mathcal{A}_{v}$. That is on $\hat{\mathcal{A}}_{v}$ two topologies were considered above the weakened $\tau_{w}^{1}$ topology and the norm $\tau_{n}$ topology.

8) Definitions. We say that a real vector space $Z$ is supplied with a scalar product if a bi-R-linear bi-additive mapping $\langle\rangle:, Z^{2} \rightarrow \boldsymbol{R}$ is given satisfying the conditions:

1) $\langle x, x\rangle \geq 0,\langle x, x\rangle=0$ if and only if $x=0$;

2) $\langle x, y\rangle=\langle y, x\rangle$;

3) $\langle a x+b y, z\rangle=a\langle x, z\rangle+b\langle y, z\rangle$ for all real numbers $a, b \in \boldsymbol{R}$ and vectors $x, y, z \in Z$.

Then an $\mathcal{A}_{r}$ vector space $X$ is supplied with an $\mathcal{A}_{r}$ valued scalar product, if a bi-R-linear bi- $\mathcal{A}_{r}$-additive mapping $\langle *, *\rangle: X^{2} \rightarrow \mathcal{A}_{r}$ is given such that

$$
(S P)\langle f, g\rangle=\sum_{j, k}\left\langle f_{j}, g_{k}\right\rangle i_{j}^{*} i_{k},
$$

where $f=f_{0} i_{0}+\cdots+f_{m} i_{m}+\cdots, f, g \in X, f_{j}, g_{j} \in X_{j}$, each $X_{j}$ is a real linear space with a real valued scalar product, $\left(X_{j},\langle *, *\rangle\right)$ is real linear isomorphic with $\left(X_{k},\langle *, *\rangle\right)$ and $\left\langle f_{j}, g_{k}\right\rangle \in \boldsymbol{R}$ for each $j, k$. The scalar product induces the norm:

$$
\|f\|:=\sqrt{\langle f, f\rangle} .
$$

An $\mathcal{A}_{r}$ normed space or an $\mathcal{A}_{r}$ vector space with $\mathcal{A}_{r}$ scalar product complete relative to its norm will be called an $\mathcal{A}_{r}$ Banach space or an $\mathcal{A}_{r}$ Hilbert space respectively.

9) Meta-Invariant Operators. If a topology $\tau$ on an $\mathcal{A}_{v}$ vector space $X$ is such that the addition of vectors and the multiplication of vectors on Cayley-Dickson numbers from $\mathcal{A}_{v}$ on the left and on the right are continuous relative to the topology $\tau$ on $X$ and the norm topology $\|*\|$ on $\mathcal{A}_{v}$, then $X$ is called a topological $\mathcal{A}_{v}$ vector space.

Let $X$ and $Y$ be topological vector spaces over the Cayley-Dickson algebra $\mathcal{A}_{v}$. An $\boldsymbol{R}$-linear $\mathcal{A}_{v}$-additive operator $C: X \rightarrow Y$ we call $\mathcal{A}_{s}$ meta-invariant for $\operatorname{card}(s)<\operatorname{card}(v)$, if a family $\left\{\Psi_{t}: t \in \Upsilon\right\}$ of CayleyDickson sub-algebras $\Psi_{t}$ isomorphic with $\mathcal{A}_{s}$ exists such that

$$
\begin{aligned}
& (M 1) \Psi_{t} \subset \mathcal{A}_{v} \text { and } \\
& (M 2) c_{\mathcal{A}_{v}}\left[\operatorname{span}_{R} \bigcup_{t \in Y} \Psi_{t}\right]=\mathcal{A}_{v} \text { and } \\
& (M 3) c l_{X}\left[\operatorname{span}_{R} \bigcup_{t \in Y}^{t} X\right]=X \text { and } \\
& (M 4) c l_{Y}\left[\operatorname{span}_{R} \bigcup_{t \in Y}^{t} Y\right]=Y \text { and } \\
& (M 5) C\left({ }^{t} X\right) \subset{ }^{t} Y
\end{aligned}
$$

for each $t \in \Upsilon$, where ${ }^{t} X$ and ${ }^{t} Y$ are vector sub-spaces over $\Psi_{t}$ in $X$ and $Y$ respectively (may be up to a continuous with its inverse isomorphism of $\mathcal{A}_{v}$ topological vector spaces as two-sided $\mathcal{A}_{v}$ modules), $\mathrm{cl}_{G} \mathrm{H}$ denotes the closure of a subset $H$ in a topological space $G$. 
In another words for $s=1$ it is complex meta-invariant, for $s=2$ or $s=3$ such operator will be called quaternion or octonion meta-invariant correspondingly.

We say that a family $\mathcal{T}$ of operators is $\mathcal{A}_{\mathrm{s}}$ metainvariant, if Conditions $(M 1-M 5)$ are fulfilled for each $C \in \mathcal{T}$ and decompositions $(M 1-M 4)$ are the same for the entire family $\mathcal{T}$.

One example, is the following. If $C \in L_{r}(X, Y)$ or $C \in L_{l}(X, Y)$ is a right or left $\mathcal{A}_{v}$ linear operator for normed spaces $X$ and $Y$ over $\mathcal{A}_{v}$ such that $C\left(X_{j}\right) \subset Y_{k}$ with $k=k(j)$ for each $j$, then

$$
C\left(X_{j} i_{j}\right)=C\left(X_{j}\right) i_{j} \subset Y_{k} i_{j}
$$

or

$$
C\left(X_{j} i_{j}\right)=i_{j} C\left(X_{j}\right) \subset i_{j} Y_{k}=Y_{k} i_{j}
$$

correspondingly. Therefore, (up to a continuous with its inverse isomorphism of $\mathcal{A}_{v}$ normed spaces as two-sided $\mathcal{A}_{v}$ modules) such operator $C$ is $\mathcal{A}_{s}$ meta-invariant for any $\operatorname{card}(s)<\operatorname{card}(v)$.

Let $X$ be a Hilbert space over the Cayley-Dickson algebra $\mathcal{A}_{v}$, then there exists an underlying Hilbert space $X_{R}$ over $\boldsymbol{R}$, i.e. a real shadow $X_{R}$. The scalar product on $X$ with values in $\mathcal{A}_{v}$ from $\$ 2.45$ induces the scalar product $(x, y):=\operatorname{Re}\langle x, y\rangle$ in $X_{\boldsymbol{R}}$ with values in $\boldsymbol{R}$.

Then for an $\mathcal{A}_{v}$-vector subspace $Y$ in $X$ the orthogonal complement $Y^{\perp}$ can be created relative to the scalar product $(x, y)$. At the same time the equation

$\hat{E}_{\mathrm{e}}(y+z)=y$ for $y \in Y, \quad z \in Y^{\perp}$ defines the $\boldsymbol{R}$-linear $(x, y):=\operatorname{Re}\langle x, y\rangle$ operator $\hat{E}_{e}$ acting on a Hilbert space $X$. That is, $\hat{E}_{e}$ is the projection on $Y$ parallel to $Y^{\perp}$. Moreover, one has $\hat{E}_{e}^{2}=\hat{E}_{e}$,

1) $Y=\left\{y: y=\hat{E}_{e}(x), x \in X\right\}=\left\{y \in X: \hat{E}_{e}(y)=y\right\}$, also

2) $Y^{\perp}=\left\{z \in X: \hat{E}_{e}(z)=0\right\}$.

Mention, that $\left(I-\hat{E}_{\mathrm{e}}\right)$ is the orthogonal projection from $X$ on $Y^{\perp}$, since $\left(Y^{\perp}\right)^{\perp}=Y$ and

$\left(I-\hat{E}_{\mathrm{e}}\right)(z+y)=z$ for each $z \in Y^{\perp}$ and $y \in Y$.

Consider the decomposition of a Hilbert space $X=X_{0} \oplus X_{1} i_{1} \oplus \cdots \oplus X_{m} i_{m} \oplus \cdots$ over $\mathcal{A}_{v}$, where $\left\{i_{0}, i_{1}, \cdots, i_{m}, \cdots\right\}$ is the family of standard generators of the Cayley-Dickson algebra $\mathcal{A}_{v}, i_{0}=1, X_{0}, \cdots, X_{m}, \cdots$ are pairwise isomorphic Hilbert spaces over $\boldsymbol{R}$.

A family of operators $E(a, *) \in L_{q}(X)$ with $a \in \mathcal{A}_{v}$ we call an $\mathcal{A}_{v}$-graded projection operator on $X$, if it satisfies conditions $(1-7)$ below:

1) $\hat{E} x=\hat{E}(x)=\hat{E}_{e}(x)$ for each $x \in X_{0}$, where

2) $\hat{E} x:=E(1, x)$ for each $x \in X$,

3) $\hat{E}_{e}^{2} x=\hat{E}_{e} x$ for each $x \in X$,

4) $E(s, x)=E(1, s x)$ for each $s \in \mathcal{A}_{v}$ and $x \in X_{0}$,

5) $\hat{E}(s x)=: \hat{E}_{s} X$ for each $s \in \mathcal{A}_{v}$ and $x \in X_{0}$,

6) $\hat{E}_{s}\left(\hat{E}_{q} x\right)=\hat{E}_{s q}(x)$ for each $s, q \in\left\{i_{0}, \cdots, i_{m}, \cdots\right\}$ and $x \in X_{0}$,

7) $\hat{E}_{i_{j}}^{*}=(-1)^{\eta(j)} \hat{E}_{i_{j}}$ on $X_{0}$ for each $j=0,1, \cdots, m, \cdots$, where $\eta(0)=0, \eta(j)=1$ for each $j \geq 1, \hat{E}_{s}^{*}$ denotes the adjoint operator.

\section{Normal Operators}

1) Definition. An operator $T$ in a Hilbert space $X$ over the Cayley-Dickson algebra $\mathcal{A}_{v}$ is called normal, if $T T^{*}=T^{*} T$.

An operator $T$ is unitary, if $T T^{*}=I$ and $T^{*} T=I$.

An operator $T$ is called symmetrical, if

$\langle T x ; y\rangle=\langle x ; T y\rangle$ for each $x, y \in \mathcal{D}(T)$.

An operator $T$ is self-adjoint, if $T^{*}=T$. Further, for $T^{*}$ it is supposed that $\mathcal{D}(T)$ is dense in $X$.

2) Remark. In $\S 1$ the more general definition than previously of a unitary operator is given without any restriction on either $\mathcal{A}_{1}$ meta-invariance or left- or right- $\mathcal{A}_{v}$-linearity.

On a space $C(B, \boldsymbol{R})$ of real-valued continuous functions $f: B \rightarrow \boldsymbol{R}$ on a Hausdorff topological space $B$ one considers a lattice structure:

1) $(f \vee g)(x)=\max \{f(x), g(x)\}$ and

2) $(f \wedge g)(x)=\min \{f(x), g(x)\}$ for each $x \in B$.

Therefore, one gets the decomposition: $f=f^{+}-f^{-}$, where $f^{+}=f \vee 0$ and $f^{-}=-(f \wedge 0)$ so that a function $f$ is the difference of two positive functions with supports

$$
\begin{aligned}
& \operatorname{supp}\left(f^{+}\right) \cap \operatorname{supp}\left(f^{-}\right)=f^{-1}(0), \\
& \operatorname{supp}(f)=c l\{x \in B: f(x) \neq 0\} .
\end{aligned}
$$

Suppose that $f, g_{1}$ and $g_{2}$ are positive or nonnegative continuous functions on $B$. Put $f_{1}=f \wedge g_{1}$ and $f_{2}=f-f_{1}$, consequently, $0 \leq f_{1} \leq g_{1}$ and $f=f_{1}+f_{2}$ so that $f_{2}$ is a positive or nonnegative continuous function. If $g_{2}(x)<f_{2}(x)=f(x)-f_{1}(x)$ for some $x \in B$, then $g_{1}(x)+g_{2}(x)<f(x)$, since $0 \leq g_{2}(x)$ and $\min \left\{f(x), g_{1}(x)\right\}=g_{1}(x)$. This contradicts the hypothesis $f \leq g_{1}+g_{2}$. Thus $f_{2} \leq g_{2}$. Therefore, the decomposition $f=f_{1}+f_{2}$ with $0 \leq f_{1} \leq g_{1}$ and $0 \leq f_{2} \leq g_{2}$ is valid, which is called Riesz' decomposition.

Consider now a space $C\left(B, \mathcal{A}_{v}\right)$ of all continuous $\mathcal{A}_{v}$ valued functions on a Hausdorff topological space $B$. An $\boldsymbol{R}$ linear $\mathcal{A}_{v}$ additive functional $p$ on $C\left(B, \mathcal{A}_{v}\right)$ we call Hermitian if $p(\tilde{f})=\overline{p(f)}$ for each $f \in C\left(B, \mathcal{A}_{v}\right)$.

This induces a lattice structure on the set $\boldsymbol{H}\left(B, \mathcal{A}_{v}\right)$ of all Hermitian functionals $t$ on $C\left(B, \mathcal{A}_{v}\right)$, since the restriction $\left.t\right|_{C(B, \boldsymbol{R})}$ is a real-valued function.

For an $\boldsymbol{R}$-linear $\mathcal{A}_{v}$ additive functional $t$ on $C\left(B, \mathcal{A}_{v}\right)$ a conjugate functional $t^{*}$ is defined by the formula

3) $t^{*}(f)=\widetilde{t(\tilde{f})}$ for each $f \in C\left(B, \mathcal{A}_{v}\right)$. 
Then the functional $t_{1}=\frac{1}{2}\left(t+t^{*}\right)$ is Hermitian and $t_{2}=\frac{1}{2}\left(t-t^{*}\right)$ is skew Hermitian, that is

$t_{2}(\tilde{f})=-t_{2}(f)$ for each $f \in C\left(B, \mathcal{A}_{v}\right)$. Therefore, the functional $t$ generates the family of Hermitian functionals ${ }_{k} t_{1}$ for each $k=0,1,2, \cdots$, where ${ }_{k} t(f):=i_{k}^{*} t\left(i_{k} f\right)$ for each $f \in C\left(B, \mathcal{A}_{v}\right)$.

Each Hermitian functional $p \in \boldsymbol{H}\left(B, \mathcal{A}_{v}\right)$ induces an $\mathcal{A}_{v}$ valued scalar product by the formula:

4) $\langle f, g\rangle:=p\left(g^{*} f\right)$.

If a topological space $B$ is compact, then

$-\|g\| 1 \leq g \leq\|g\| 1$. If $p$ is a Hermitian state, then

5) $|p(1 g)|^{2} \leq p\left(|g|^{2}\right) p\left(1^{2}\right) \leq 1$ due to Cauchy-Schwartz' inequality.

Thus Hermitian state $p$ is continuous and has norm 1 .

3) Lemma. Let $T \in L_{q}(X)$ be a normal $\mathcal{A}_{r}$ metainvariant operator for a Hilbert space $X$ over the CayleyDickson algebra $\mathcal{A}_{v}$ with $2 \leq r \leq \min (3, v)$. Let also $A$ be a minimal von Neumann algebra generated by $T, T^{*}$ and I over $\mathcal{A}_{v}$. Suppose that $\Psi_{t}$ is a sub-algebra in $\mathcal{A}_{v}$ isomorphic with $\mathcal{A}_{r}$ for each $t \in \Upsilon$ and

$N_{0, t}, \cdots, N_{2^{r}-1, t}$ are generators of $\Psi_{t}$ (see $\S 2.9$ ). Then the restricted sub-algebra

${ }^{t} \boldsymbol{A}:={ }^{t} A_{0} N_{0, t} \oplus \cdots \oplus{ }^{t} A_{2^{r}-1} N_{2^{r}-1, t}$ is associative for $r=2$ and alternative for $r=3$ for each $t \in \Upsilon$.

Proof. In view of Theorem 2.22 [12] an algebra $A$ is quasi-commutative. Compositions $T^{n} \circ T^{m}$ are considered as point-wise set-theoretic compositions of mappings so that $T^{n} \circ T^{m} x=T^{n+m}=T(T(\cdots(T x) \cdots))$ is the $n+m$ times composition of $T$ with itself for each $x \in X$, where $n, m \in N$ are natural numbers. Moreover, $I x=x$ for each $x \in X$. Then consider $\tau:=\left.T\right|_{t_{X}}$ as a formal variable so that $\tau^{0}=I$. This variable is power associative $\tau^{n} \tau^{m}=\tau^{n+m}$ for all $n, m \in N$, since $T$ is $\mathcal{A}_{r}$ metainvariant. Moreover, $\tau$ and $\tau^{*}$ commute, $\tau^{*} \tau=\tau \tau^{*}$, since $T$ is the normal operator, $T T^{*}=T^{*} T$.

Therefore, the minimal algebra $\operatorname{alg}_{\Psi_{t}}\left\{\tau, \tau^{*}, I\right\}$ over $\Psi_{t}$ generated by $\tau, \tau^{*}$ and $I$ consists of formal polynomials in variables $\tau, \tau^{*}$ with coefficients from $\Psi_{t}$. This algebra is $\boldsymbol{R}$ linear and left and right module over $\Psi_{t}$. Relative to addition and multiplication of polynomials the algebra $\operatorname{alg}_{\Psi_{t}}\left\{\tau, \tau^{*}, I\right\}$ is associative for $r=2$ and alternative for $r=3$, since the quaternion skew field $\mathcal{A}_{2}=\boldsymbol{H}$ is associative and the octonion algebra $\mathcal{A}_{3}=\boldsymbol{O}$ is alternative and $\Psi_{t}$ is isomorphic with $\mathcal{A}_{r}$ for each $t \in \Upsilon$.

Consider the algebra $\operatorname{alg}_{\Psi_{t}}\left\{\tau, \tau^{*}, I\right\}$ relative to the topology inherited from the von Neumann algebra ${ }^{t} \boldsymbol{A}$ (see also Definitions 2.22.1 and Theorem 2.22 [12]).

The completion of $\operatorname{alg}_{\Psi_{t}}\left\{\tau, \tau^{*}, I\right\}$ gives the von Neu- mann algebra ${ }^{t} \boldsymbol{A}$ up to isomorphism of algebras. The operations of addition and multiplication are continuous relative to a norm in a $C^{*}$-algebra. Thus the restricted sub-algebra ${ }^{t} \boldsymbol{A}$ is associative for $r=2$ and alternative for $r=3$ for each $t \in \Upsilon$.

4) Theorem. Suppose that $T \in L_{q}(X)$ is a self-adjoint $\mathcal{A}_{r}$ meta-invariant operator on a Hilbert space $X$ over the Cayley-Dickson algebra $\mathcal{A}_{v}$ with $2 \leq v$ and $2 \leq r \leq \min (3, v), \boldsymbol{A}$ is a von Neumann algebra containing T. Then there is a family $\left\{{ }_{b} \hat{E}: b \in \boldsymbol{R}\right\} \subset \boldsymbol{A}$ of $\mathcal{A}_{v}$ graded projection operators on $X$ such that

1) ${ }_{b} \hat{E}=0$ for $b<-\|T\|$, while ${ }_{b} \hat{E}=I$ for $b \geq\|T\|$;

2) ${ }_{b} \hat{E} \leq_{t} \hat{E}$ for $b \leq t$;

3) ${ }_{b} \hat{E}=\Lambda_{t>b t} \hat{E}$;

4) $\quad T_{b} \hat{E} \leq(b I){ }_{b} \hat{E} \quad$ and $\quad(b I)\left(I-{ }_{b} \hat{E}\right) \leq T\left(I-{ }_{b} \hat{E}\right)$ for each $b \in \boldsymbol{R}$;

5) $T=\int_{-\|T\|}^{\|T\|} b \mathrm{~d}_{b} \hat{E}$ and this integral is the limit of Riemann sums converging relative to the operator norm.

Considering a restricted algebra

$\boldsymbol{A}_{\mathcal{A}_{r}}:=\boldsymbol{A}_{0} i_{0} \oplus \cdots \oplus \boldsymbol{A}_{2^{r}-1} i_{2^{r}-1}$ isomorphic to $C\left(\Lambda, \mathcal{A}_{r}\right)$ with an extremely disconnected compact Hausdorff topological space $\Lambda$ let $f$ and ${ }_{b} e$ in $C\left(\Lambda, \mathcal{A}_{r}\right)$ correspond to $\left.T\right|_{Y}$ and $\left.{ }_{b} E\right|_{Y}$ in $\boldsymbol{A}$ respectively, then ${ }_{b} e$ is the characteristic function of the largest clopen subset $\Lambda_{b}$ on which $f$ takes values not exceeding $b$, where $Y=X_{0} i_{0} \oplus \cdots \oplus X_{2^{r}-1} i_{2^{r}-1}$.

Proof. An operator $T$ is $\mathcal{A}_{r}$-meta-invariant, consequently, a von Neumann subalgebra $A_{\mathcal{A}_{r}}$ over $\mathcal{A}_{r}$ contains the restriction $\left.T\right|_{Y}$ of $T$ on $Y$ up to an isomorphism of von Neumann algebras over $\mathcal{A}_{r}$, where

$2 \leq r \leq \min (3, v)$.

In accordance with Theorem 2.22 [12] we have that an algebra $A_{\mathcal{A}_{r}}$ is isomorphic with $C\left(\Lambda, \mathcal{A}_{r}\right)$ for some extremely disconnected compact Hausdorff topological space $\Lambda$.

Recall that a subset $B$ of a topological space $W$ is called clopen, if it is closed and open simultaneously, $c l(B)=\operatorname{Int}(B)=B$.

If a function $f$ corresponds to an operator $T$, then a set $\Lambda_{b}:=\Lambda \backslash c l\left[f^{-1}(b, \infty)\right]$ is clopen in $\Lambda$. On this set the function $f$ has values not exceeding $b$. If $V$ is another clopen subset so that

$f(V) \subset(-\infty, b]:=\{x \in \boldsymbol{R}: x \leq b\}$, then $V \subset \Lambda \backslash f^{-1}(b, \infty)$ and $f^{-1}(b, \infty) \subset \Lambda \backslash V$. On the other hand, the set $\Lambda \backslash V$ is clopen, consequently, $c l\left[f^{-1}(b, \infty)\right] \subset \Lambda \backslash V$. Therefore $\Lambda_{b}$ is the largest clopen subset in $\Lambda$ so that $f\left(\Lambda_{b}\right) \subset(-\infty, b]$.

The characteristic function ${ }_{b} e$ of $\Lambda_{b}$ by its definition takes value 1 on $\Lambda_{b}$ and zero on its complement $\Lambda \backslash \Lambda_{b}$. It is continuous ${ }_{b} e \in C(\Lambda, \boldsymbol{R})$, since the subset $\Lambda_{b}$ is clopen in $\Lambda$. Thus an operator ${ }_{b} \hat{E}$ in $A$ corre- 
sponding to ${ }_{b} e$ is a projection, ${ }_{b} \hat{E}: X \rightarrow X,{ }_{b} \hat{E}^{2}={ }_{b} \hat{E}$. Then to ${ }_{b} e i_{j}=i_{j b} e$ an operator ${ }_{b} \hat{E}_{i_{j}}={ }_{b} \hat{E}\left(i_{j} I\right)=\left(i_{j} I\right)_{b} \hat{E}$ on $X_{j}$ corresponds, where $j=0,1,2, \cdots$. Over the CayleyDickson algebra $\mathcal{A}_{v}$ the projection ${ }_{b} \hat{E}$ induces an $\mathcal{A}_{v}$ graded projection in accordance with $\$ 2.9$, since $X=X_{0} i_{0} \oplus \cdots \oplus X_{m} i_{m} \oplus \cdots$.

Statements $(1,2,4)$ are evident from the definition of the subsets $\Lambda_{b}$ and the properties of the isomorphism of $\boldsymbol{A}_{\mathcal{A}_{r}}$ with $C\left(\Lambda, \mathcal{A}_{r}\right)$. Indeed, the function $f$ is continuous and

$$
f\left(c l\left[f^{-1}(b, \infty)\right]\right) \subset[b, \infty):=\{x \in \boldsymbol{R}: x \geq b\} .
$$

For proving Statement (3) we mention that ${ }_{b} e=\inf _{t>b} e$, since the topological space $\Lambda$ is extremely disconnected and each $\Lambda_{b}$ is clopen in $\Lambda$. This implies that ${ }_{b} \hat{E}$ is the infimum of the family

$\left\{{ }_{t} \hat{E}: t>b\right\}$, consequently, ${ }_{b} \hat{E}=\Lambda_{t>b} t \hat{E}$, since

$\Lambda_{t>b t} \hat{E} \in L_{q}(X)$ in accordance with Propositions 2.22.7 and 2.22.9 and Corollary 2.22.13, Definition 2.23 and Theorem 2.24 [12] with ${ }_{b} \hat{E}=I-\hat{E}(b, \infty)$.

It remains to prove Statement (5). Take $b_{0}<-\|T\|$ and a partition $\left\{b_{0}, b_{1}, \cdots, b_{n}\right\}$ of the segment $\left[b_{0},\|T\|\right]$ so that $b_{0}<b_{1}<\cdots<b_{n}=\|T\|$. If $\left[b_{j-1}, b_{j}\right] \cap s p(T) \neq \varnothing^{n}$ let $c_{j}$ be a point of this intersection, otherwise let $c_{j}=b_{j-1}$ when the intersection is empty, $\left[b_{j-1}, b_{j}\right] \cap s p(T)=\varnothing$. Therefore,

$f^{-1}\left(\left[b_{j-1}, b_{j}\right]\right)=\varnothing$ when this intersection is empty, since a spectrum $\operatorname{sp}(T)=f(\Lambda)$ of $T$ is a range of $f$, consequently, $f^{-1}\left(b_{j-1}, \infty\right)=f^{-1}\left(b_{j}, \infty\right)$ and ${ }_{b_{j-1}} e==_{b_{j}} e$.

We call two characteristic functions $\chi_{A}$ and $\chi_{B}$ orthogonal, if $\chi_{A} \chi_{B}=0$ identically, that is $A \cap B=\varnothing$.

Thus characteristic functions $\left({ }_{b_{j}} e-{ }_{b_{j-1}} e\right)$ are mutually orthogonal for different values of $j$. We consider the function $h=\sum_{j=1}^{n} c_{j}\left(b_{j} e-{ }_{b_{j-1}} e\right)$. This induces the disjoint partition $\Psi_{j}=\Lambda_{b_{j}} \backslash \Lambda_{b_{j-1}}$ with $X_{b_{0}}=\varnothing$, that is $\Lambda=\bigcup_{j=1}^{n} \Psi_{j}$ and $\Psi_{j} \cap \bigcap \Psi_{k}=\varnothing$ for each $j \neq k$. If $t \in \Psi_{j}$, then $h(t)=c_{j}$ and $b_{j-1} \leq f(t) \leq b_{j}$, hence

6) $\|f-h\| \leq \max _{j}\left|b_{j}-b_{j-1}\right|$.

We consider Riemann integral sums

$$
\boldsymbol{S}\left(b_{0}, \cdots, b_{n} ; c_{1}, \cdots, c_{n}\right):=\sum_{j=1}^{n} c_{j}\left({ }_{b_{j}} \hat{E}-{ }_{b_{j-1}} \hat{E}\right) .
$$

If $\theta: \boldsymbol{A}_{\mathcal{A}_{r}} \rightarrow C\left(\Lambda, \mathcal{A}_{r}\right)$ is an isomorphism of quasicommutative $C^{*}$ algebras, then

7) $\left.\theta \boldsymbol{S}\left(b_{0}, \cdots, b_{n} ; c_{1}, \cdots, c_{n}\right)\right|_{Y}=h$.

On the other hand,

$$
\left\|\left.\boldsymbol{S}\left(b_{0}, \cdots, b_{n} ; c_{1}, \cdots, c_{n}\right)\right|_{Y}\right\|=\left\|\left.\boldsymbol{S}\left(b_{0}, \cdots, b_{n} ; c_{1}, \cdots, c_{n}\right)\right|_{X}\right\|,
$$

since the operator $T$ is $\mathcal{A}_{r}$ meta-invariant and hence ${ }_{b} \hat{E}$ is $\mathcal{A}_{r}$ meta-invariant as well for each $b$, since ${ }_{b} e \in C(\Lambda, \boldsymbol{R})$ and ${ }_{b} \hat{E}\left(X_{0}\right) \subset X_{0}$ and ${ }_{b} \hat{E}$ is a graded projection operator (see $\$ 2.9$ ). Therefore, Formulas (6), (7) imply Statement (5) in the sense of norm convergence in $L_{q}(X)$.

5) Definition. A family of $\mathcal{A}_{v}$ graded projections ${ }_{b} \hat{E}$ indexed by $b \in \boldsymbol{R}$ satisfying Conditions $(2,3)$ of the preceding theorem and two conditions below
1) $\wedge_{b \in \boldsymbol{R} b} \hat{E}=0$ and
2) $\vee_{b \in R b} \hat{E}=I$

is called an $\mathcal{A}_{v}$ graded spectral resolution of the identity. An $\mathcal{A}_{v}$ graded spectral resolution of the identity is called bounded, when two finite numbers $-\infty<a<c<\infty$ exist such that ${ }_{b} \hat{E}=0$ for every $b<a$ and ${ }_{b} \hat{E}=I$ for each $b>c$. Otherwise we say that a resolution of the identity is unbounded.

6) Definitions. $A^{*}$-homomorphism $\phi$ of a $C^{*}$-algebra $\boldsymbol{A}$ into $L_{q}(X)$ is called a representation of $\boldsymbol{A}$ on a Hilbert space $X$ over the Cayley-Dickson algebra $\mathcal{A}_{v}$, that is $\phi(a f+b g)=a \phi(f)+b \phi(g)$ and $\phi(f g)=\phi(f) \phi(g)$ and $\phi\left(f^{*}\right)=[\phi(g)]^{*}$ for all $a, b \in \boldsymbol{R}$ and $f, g \in \boldsymbol{A}$, if $\boldsymbol{A}$ contains a unit element $e \in \boldsymbol{A}$, then $\phi(e)=I$.

If additionally $\phi$ is one-to-one, then such a mapping will be called a faithful representation of $\boldsymbol{A}$ onto $\boldsymbol{B}$, where $\boldsymbol{B}:=\phi(\boldsymbol{A}) \subset L_{q}(X)$.

A representation $\phi$ is said to be essential if the union of ranges of $\mathcal{A}_{v}$ graded projection operators (elements) of $\boldsymbol{A}$ in the image $L_{q}(X)$ is the unit operator $I$.

Let $C_{0}\left(\boldsymbol{R}, \mathcal{A}_{r}\right)$ denote the subspace of all continuous vanishing at infinity functions $f: \boldsymbol{R} \rightarrow \mathcal{A}_{r}$ so that

$$
\lim _{|x| \rightarrow \infty} f(x)=0 \text { and }\|f\|=\sup _{x \in \boldsymbol{R}}|f(x)|<\infty .
$$

7) Theorem. Suppose that $\left\{{ }_{b} \hat{F}: b \in \boldsymbol{R}\right\}$ is an $\mathcal{A}_{v}$ graded resolution of the identity and $T$ is a bounded self-adjoint operator $T \in L_{q}(X)$ such that $T_{b} \hat{F} \leq b_{b} \hat{F}$ and $b\left(I-{ }_{b} \hat{F}\right) \leq T\left(I-{ }_{b} \hat{F}\right)$ for every $b \in \boldsymbol{R}$ or $T=\int^{a} b \mathrm{~d}_{b} \hat{F}$ for each $a>a_{0}$, where $a_{0}>0$ is a marked positive number, $2 \leq v$. Then $\left\{{ }_{b} \hat{F}: b \in \boldsymbol{R}\right\}$ is the resolution of the identity for $T \in \boldsymbol{A}$, where $\boldsymbol{A}$ is a quasi-commutative von Neumann algebra generated by $T$ and I over the Cayley-Dickson algebra $\mathcal{A}_{v}$,

$\boldsymbol{A}=\widetilde{\operatorname{alg}_{\mathcal{A}_{v}}}\{T, I\}, \tilde{B}$ denotes a completion of a sub-algebra $B$ in $L_{q}(X)$ relative to the weak operator topology.

Proof. The inequality $T_{b} \hat{F} \leq b_{b} \hat{F}$ implies that $T_{b} \hat{F}$ is self-adjoint. Therefore, restrictions $\left.T\right|_{X_{0}}$ and $\left.{ }_{b} \hat{F}\right|_{X_{0}}$ commute on $X_{0}$ for each $b$. Hence the algebra 
$\boldsymbol{A}=\operatorname{alg}_{\mathcal{A}_{v}}\{T, I\}$ is quasi-commutative. In view of Theorem 3 the restricted algebra $\boldsymbol{A}_{\mathcal{A}_{r}}$ is isomorphic with $C\left(\Lambda, \mathcal{A}_{r}\right)$, where $\Lambda$ is an extremely disconnected compact Hausdorff space and $2 \leq r \leq \min (3, v)$.

If ${ }_{b} \hat{E}$ is a resolution of the identity for an operator $T \in \boldsymbol{A}$, a function $f$ in $C\left(\Lambda, \mathcal{A}_{v}\right)$ corresponds to $T$, while ${ }_{b} e \in C\left(\Lambda, \mathcal{A}_{v}\right)$ corresponds to ${ }_{b} \hat{E}$, then ${ }_{b} e$ is the characteristic function of the largest clopen subset $\Lambda_{b}$ in $\Lambda$ on which $f$ takes values not exceeding $b$. On the other hand, if a function ${ }_{b} f \in C\left(\Lambda, \mathcal{A}_{v}\right)$ corresponds to ${ }_{b} \hat{F}$, then ${ }_{b} f$ is the characteristic function of a clopen subset $\Theta_{b}$ in $\Lambda$ on which $f$ takes values not exceeding $b$, since ${ }_{b}$ ff $\leq b_{b} f$, consequently, $\Theta_{b} \subset \Lambda_{b}$.

The equality ${ }_{b} \hat{F}=\Lambda_{t>b}{ }_{t} \hat{F}$ implies that $\Theta_{b}$ is the largest clopen subset contained in $\bigcap_{t>b} \Theta_{t}$. By the conditions of this theorem $t\left(I-{ }_{t} \hat{F}\right) \leq T\left(I-{ }_{b} \hat{F}\right)$ and

$\Lambda \backslash \Theta_{b} \subset c l\left[f^{-1}(b, \infty)\right]$ for any $t>b$. Hence $t \leq f(p)$ for each $p \in \Lambda \backslash \Theta_{t}$, consequently, $\Lambda_{b} \subset \Theta_{t}$ for each $t>b$ and inevitably $\Lambda_{b}$ is a clopen subset contained in $\bigcap_{t>b} \Theta_{t}$. Therefore, $\Lambda_{b} \subset \Theta_{b}$, since $\Theta_{b}$ is the largest clopen subset contained in $\bigcap_{t>b} \Theta_{t}$. Thus $\Theta_{b}=\Lambda_{b}$ and ${ }_{b} \hat{F}={ }_{b} \hat{E}$ for each $b \in \boldsymbol{R}$.

The resolution of the identity for the operator $T$ in $A$ satisfies Condition (4) of Theorem 4 and $A \subset L_{q}(X)$.

Now, if

$$
T=\int_{-a}^{a} b \mathrm{~d}_{b} \hat{F}
$$

for each $a>a_{0}$, where $a_{0}>0$ is a marked positive number, $2 \leq v$, we take for each $\epsilon>0$ an arbitrary partition $\left\{b_{0}, \cdots, b_{n}\right\}$ of the segment $[-a, a]$ so that $\|T-Q\|<\epsilon$ for an operator $Q=\sum_{j=1}^{n} c_{j}\left({ }_{b_{j}} \hat{F}-_{b_{j-1}} \hat{F}\right)$ with $c_{j} \in\left[b_{j-1}, b_{j}\right]$ for each $j$. This implies that $\left\|(T-Q)_{b} \hat{F}\right\|<\epsilon$ and

$$
\begin{aligned}
Q_{b_{k}} \hat{F} & =\sum_{j=1}^{k} c_{j}\left(b_{j} \hat{F}-{ }_{b_{j-1}} \hat{F}\right) \\
& \leq \sum_{j=1}^{k} b_{k}\left({ }_{b_{j}} \hat{F}-{ }_{b_{j-1}} \hat{F}\right) \\
& =b_{k}\left({ }_{b_{k}} \hat{F}-{ }_{-a} \hat{F}\right) \leq b_{k b_{k}} \hat{F},
\end{aligned}
$$

consequently, $T_{b_{k}} \hat{F} \leq b_{k b_{k}} \hat{F}$. We have also

$$
\left\|(T-Q)\left(I-{ }_{b_{k}} \hat{F}\right)\right\|<\epsilon
$$

and

$$
\begin{aligned}
Q\left(I-{ }_{b_{k}} \hat{F}\right) & =\sum_{j=k+1}^{n} c_{j}\left(b_{j} \hat{F}-{ }_{b_{j-1}} \hat{F}\right) \\
& \geq \sum_{j=k+1}^{n} b_{k}\left({ }_{b_{j}} \hat{F}-{ }_{b_{j-1}} \hat{F}\right) \\
& =b_{k}\left({ }_{a} \hat{F}-{ }_{b_{k}} \hat{F}\right),
\end{aligned}
$$

consequently, $\quad T\left(I-{ }_{b_{k}} \hat{F}\right) \geq b_{k}\left({ }_{a} \hat{F}-{ }_{b_{k}} \hat{F}\right)$ for every $a>a_{0}$. Take a limit in the strong operator topology with $a$ tending to the infinity: $\lim _{a \rightarrow \infty} \hat{F}=I$, consequently, $T\left(I-{ }_{b} \hat{F}\right) \geq b\left(I-{ }_{b} \hat{F}\right)$ for each $b \in \boldsymbol{R}$, since $\epsilon>0$ is arbitrary, where ${ }_{b} \hat{F}={ }_{b} \hat{E}$ for every $b \in \boldsymbol{R}$ in accordance with the first part of the proof.

8) Lemma. Let $T$ be an $\mathcal{A}_{r}$ meta-invariant operator on an $\mathcal{A}_{v}$ vector space $X$ and let $0 \leq r<u<v$. Then $T$ is $\mathcal{A}_{u}$ meta-invariant.

Proof. The Cayley-Dickson algebra $\mathcal{A}_{u}$ has also the structure of the $\mathcal{A}_{r}$ vector space for $0 \leq r<u$, since $\mathcal{A}_{r}$ is the sub-algebra in $\mathcal{A}_{u}$. Therefore, the decomposition from $\$ 2.9$ satisfying Conditions $(M 1-M 5)$ generates analogous decomposition fulfilling Conditions (M1-M5) over $\mathcal{A}_{u}$ as well relative to which $T$ is $\mathcal{A}_{u}$ meta-invariant.

9) Theorem. Let $\left\{{ }_{b} \hat{E}: b \in[-a, a]\right\}$ be a bounded graded resolution of the identity on a Hilbert space $X$ over the Cayley-Dickson algebra $\mathcal{A}_{v}, 2 \leq v$,

$0<a<\infty,{ }_{b} \hat{E}=0$ for each $b<-a$ and ${ }_{b} \hat{E}=I$ for each $b>a$. Then the integral $\int_{a}^{a} t \mathrm{~d}_{t} \hat{E}$ converges to $a$ self-adjoint operator $T$ on $X$ so that $\|T\| \leq a$ and

$\left\{{ }_{b} \hat{E}: b \in[-a, a]\right\}$ is the spectral resolution of such operator $T$.

Proof. Take any partitions $\left\{b_{0}, \cdots, b_{n}\right\}=\alpha$ and $\left\{q_{0}, \cdots, q_{m}\right\}=\beta$ of the segment $[-a, a]$ of diameters

$$
0<|\alpha|=\max _{j=1, \cdots, n}\left(b_{j}-b_{j-1}\right)<\infty
$$

and

$$
0<|\beta|=\max _{j=1, \cdots, m}\left(q_{j}-q_{j-1}\right)<\infty
$$

and denote by $\varnothing=\left\{t_{0}, \cdots, t_{l}\right\}=\alpha \bigcup \beta$ their common refinement. Then one gets

$$
\left\|\sum_{j=1}^{n} c_{j}\left({ }_{b_{j}} \hat{E}-{ }_{b_{j-1}} \hat{E}\right)-\sum_{s=1}^{l} g_{s}\left({ }_{b_{s}} \hat{E}-{ }_{t_{s-1}} \hat{E}\right)\right\| \leq|\alpha|
$$

and

$$
\left\|\sum_{j=1}^{n} u_{j}\left({ }_{q_{j}} \hat{E}-{ }_{q_{j-1}} \hat{E}\right)-\sum_{s=1}^{l} g_{s}\left({ }_{t_{s}} \hat{E}-{ }_{t_{s-1}} \hat{E}\right)\right\| \leq|\beta|,
$$

where

$$
c_{j} \in\left[b_{j-1}, b_{j}\right], g_{s} \in\left[t_{s-1}, t_{s}\right], u_{j} \in\left[q_{j-1}, q_{j}\right],
$$

consequently,

$$
\text { 1) } \begin{aligned}
& \left\|\sum_{j=1}^{n} c_{j}\left({ }_{b_{j}} \hat{E}-{ }_{b_{j-1}} \hat{E}\right)-\sum_{s=1}^{m} u_{s}\left({ }_{q_{s}} \hat{E}-{ }_{q_{s-1}} \hat{E}\right)\right\| \\
& \leq|\alpha|+|\beta| .
\end{aligned}
$$

Consider the family of partitions $\left\{b_{0}, \cdots, b_{n}\right\}$ of the segment $[-a, a]$ ordered by inclusion. The algebra $L_{q}(X)$ is complete relative to the operator norm topology. Therefore, a Cauchy net of finite integral sums $\sum_{j=1}^{n} c_{j}\left({ }_{b_{j}} \hat{E}-{ }_{b_{j-1}} \hat{E}\right)$ converges in $L_{q}(X)$, $\lim _{|\alpha| \rightarrow 0} \sum_{j=1}^{n} C_{j}\left(b_{j} \hat{E}-{ }_{b_{j-1}} \hat{E}\right)=T \in L_{q}(X)$ and $T^{*}=T$, 
since $c_{j} \in \boldsymbol{R}$ for each $j$ and $\alpha$.

Theorem 7 states that $\left\{{ }_{b} \hat{E}: b \in[-a, a]\right\}$ is the $\mathcal{A}_{v}$ graded spectral resolution of $T$.

In the space of continuous functions on a compact Hausdorff topological space with values in $\mathcal{A}_{v}$ a family of polynomials is everywhere dense in accordance with the Stone-Weierstrass theorem (see $\S \S I .2 .3$ and I.2.7 [13]), since $\bigcup_{s<\min \left(\omega_{0}, v\right), \theta_{s}} \theta_{s}\left(A_{s}\right)$ is everywhere dense in $\mathcal{A}_{v}$, where $\theta_{s}$ is a family of all possible embeddings of $\mathcal{A}_{s}$ into $\mathcal{A}_{v}, \omega_{0}$ denotes the first countable ordinal, $\operatorname{card}\left(\omega_{0}\right)=\aleph_{0} \quad$ (see [14]). The real field $\boldsymbol{R}$ is the center $Z\left(\mathcal{A}_{v}\right)$ of the Cayley-Dickson algebra so that each polynomial $P_{n}(t)$ of a variable $t \in \boldsymbol{R}$ with values in $\mathcal{A}_{v}$, has the form

$P_{n}(t)=\sum_{j=0}^{n} t^{j} a_{j}=\sum_{j=0}^{n} a_{j} t^{j}$, where $a_{j} \in \mathcal{A}_{v}$ are constants. In this calculus we have

$$
f(T)=\int_{-a}^{a}\left(\mathrm{~d}_{t} \hat{E}\right) \cdot f(t)
$$

for each $f \in C\left([-a, a], \mathcal{A}_{v}\right)$. Particularly, if $f$ is a nonnegative function on $[-a, a]$, then $f(T)$ is a positive operator. Therefore, $T$ is the difference of two positive operators $T={ }_{1} T-{ }_{2} T$, where $T=\int_{0}^{a} t \mathrm{~d}_{t} \hat{E}$ and ${ }_{2} T=\lim _{\epsilon \downarrow 0} \int_{-a}^{-\epsilon}(-t) \mathrm{d}_{t} \hat{E}$. These operators ${ }_{1} T$ and ${ }_{2} T$ commute, since they are presented by the corresponding integrals, which have integration over non-intersecting sets so that the graded projectors $\int_{0}^{a} \mathrm{~d}_{t} \hat{E}$ and $\lim _{\epsilon \downarrow} \int_{-a}^{-\epsilon}(-t) \mathrm{d}_{t} \hat{E}$ commute (see $\$ 2.73$ and $\$ 2.74$ ). Their sum ${ }_{1} T+{ }_{2} T$ is the positive operator, consequently, $\mathcal{A}_{r}$ meta-invariant for $2 \leq r \leq \min (3, v)$ (see $\$ 2.9$ and Lemma 8).

Consider the restricted algebra $\boldsymbol{A}_{\mathcal{A}_{r}}$ and isomorphic to it algebra $C\left(\Lambda, \mathcal{A}_{r}\right)$ and with $2 \leq r \leq \min (3, v)$, where $\Lambda$ is a compact Hausdorff extremely disconnected topological space, $A=\widetilde{\operatorname{alg}_{\mathcal{A}_{v}}}\{T, I\}$. The conditions $0<a<\infty,{ }_{b} \hat{E}=0$ for each $b<-a$ and ${ }_{b} \hat{E}=I$ for each $b>a$ imply that a function $w$ in $C\left(\Lambda, \mathcal{A}_{r}\right)$ representing the operator $T$ has range in $[-a, a]$, consequently, $\|T\| \leq a$.

10) Theorem. Suppose that $T$ is a unitary $\mathcal{A}_{r}$ metainvariant operator on an $\mathcal{A}_{v}$ Hilbert space $X, 2 \leq v<\infty$, $1 \leq r \leq \min (3, v)$, while $\boldsymbol{A}$ is the quasi-commutative von Neumann algebra generated by $T$ and $T^{*}$.

1) Then $T$ is a norm limit of finite linear combinations of mutually orthogonal projections in $\mathbf{A}$ with coefficients in $s p(T)$.

2) If additionally $r=1$ and $T$ is either right or left $\mathcal{A}_{v}$ linear operator on $X$, then a positive operator $K$ and a purely imaginary Cayley-Dickson number $M \in \mathcal{A}_{v}$, $\operatorname{Re}(M)=0,|M|=1$, exist such that $\|K\| \leq 1$ and
$T=\exp (2 \pi M K)$.

Proof. 1) Each unitary operator is normal. It preserves an $\mathcal{A}_{v}$ scalar product on $X:\langle T x, T y\rangle=\langle x, y\rangle$ for each $x, y \in \mathcal{A}_{v}$.

Consider the von Neumann algebra over $\mathcal{A}_{v}$ generated by $T$ and $T^{*}$. In view of Theorem 2.22 [12] and Lemma 3 the restricted algebra $\boldsymbol{A}_{\mathcal{A}_{r}}$ is isomorphic with $C\left(\Lambda, \mathcal{A}_{r}\right)$, where $\Lambda$ is a compact extremely disconnected topological Hausdorff space. The algebra $\boldsymbol{A}_{\mathcal{A}_{r}}$ is isomorphic with $\boldsymbol{A}_{\Psi_{t}}$ for each $t \in \Upsilon$ (see also $\$ 2.9$ ).

Recall the following. A closed subset $Q$ of a topological space $P$ is called functionally closed if $Q=\xi^{-1}(0)$ for some continuous function $\xi: P \rightarrow[0,1] \subset \boldsymbol{R}$. A subset $U$ of a topological space $P$ is called functionally open, if it is the complement of some functionally closed subset $Q$, i.e. $U=P \backslash Q$. A covering of a topological space $P$ by functionally open subsets is called a functionally open covering. It is said that a topological space $P$ is strongly zero-dimensional, if $P$ is a non-void Tychonoff space (completely regular), i.e. $T_{3 \frac{1}{2}}$, and each functionally open covering $\left\{U_{j}: j=1, \cdots, k\right\}$ has a finite disjoint open covering $\left\{V_{l}: l=1, \cdots, m\right\}$ refining $\left\{U_{j}: j\right\}$, that is $\bigcup_{l=1}^{m} V_{l}=P, V_{l} \cap V_{s}=\varnothing$ for each $l \neq s$, for every $V_{l}$ an open subset $U_{j}$ with $j=j(l)$ exists such that $V_{l} \subset U_{j(l)}$. This means that this covering $\left\{V_{l}: l\right\}$ consists of clopen subsets $V_{l}$ in $P$.

Theorem 6.2.25 [14] says that each non-void extremely disconnected Tychonoff space is strongly zerodimensional.

Therefore, to the restricted operator $\left.T\right|_{Y}$ up to an isomorphism of $C^{*}$ algebras a function $f \in C\left(\Lambda, \mathcal{A}_{r}\right)$ corresponds such that

$$
\text { 3) } f(x)=\exp (2 \pi M(x) g(x)) \text {, }
$$

where $g(x) \in \boldsymbol{R}$ and

$$
M(x) \in \mathcal{S}_{r}:=\left\{z \in \mathcal{A}_{r}:|z|=1, \operatorname{Re}(z)=0\right\}
$$

for each $x \in \Lambda, g, M \in C\left(\Lambda, \mathcal{A}_{r}\right)$ (see $\S 3$ in [16] or $\S$ I.3 [13]). The Hilbert subspace

$Y=X_{0} i_{0} \oplus \cdots \oplus X_{2^{r}-1} i_{2^{r}-1}$ is isomorphic with ${ }^{t} X$ for each $t \in \Upsilon$. Then $\operatorname{sp}\left(\left.T\right|_{Y}\right) \subset \mathcal{A}_{r}$. Generally to $\left.T\right|_{t_{X}}$ a function $f_{t} \in C\left(\Lambda^{t}, \Psi_{t}\right)$ corresponds with $s p\left(\left.T\right|_{t_{X}}\right) \subset \Psi_{t}$, where $\Lambda^{t}$ is a topological space homeomorphic with $\Lambda$. If $\theta_{t}: \Lambda \rightarrow \Lambda^{t}$ is such homeomorphism, then $f_{t}\left(\theta_{t}(u)\right)=f(u)$ for each $u \in \Lambda$.

Certainly it is sufficient to take a function $g$ with values in $[0,1]$, since the exponential function is periodic: $\exp (2 \pi M(t+n))=\exp (2 \pi M t)$ for each real number $t \in \boldsymbol{R}$ and an integer $n \in \boldsymbol{Z}$ and a purely imaginary 
number $M \in \mathcal{S}_{v}$ in the unit sphere. Therefore, a positive operator $K$ on $X$ corresponds to this function $g$.

The operator $K$ defines an $\mathcal{A}_{v}$ graded resolution $\left\{{ }_{b} \hat{E}: b \in[0,1]\right\}$ of the identity (see Theorem 4) and

4) $K=\int_{0}^{1} b \mathrm{~d}_{b} \hat{E}$.

Therefore, $\|K\| \leq 1$. We put $\Lambda_{b}=\varnothing$ for $b<0, \Lambda_{b}=\Lambda$ for $b \geq 1$, let $\Lambda_{b}$ be the largest clopen subset in $\Lambda$ on which the function $g$ has values not exceeding $b \in[0,1)$.

Let ${ }_{b} e$ be zero for $b<0,{ }_{b} e=1$ for $b \geq 1$, and let ${ }_{b} e$ be the characteristic function of $\Lambda_{b}$ for $b \in[0,1)$. Each clopen subset $V$ in $\bigcap_{u>b} \Lambda_{u}$ is contained in $\Lambda_{b}$, since $g$ takes values on $V$ in $\Lambda_{u}$ for each $u>b$, consequently, $g(V) \subset \Lambda_{b}$. This implies that ${ }_{b} e$ is the infimum of the family $\left\{{ }_{u} e: u>b\right\}$. This induces a bounded $\mathcal{A}_{v}$ graded resolution $\left\{{ }_{b} \hat{E}: b \in[0,1]\right\}$ of the identity, where ${ }_{b} e$ corresponds to ${ }_{b} \hat{E}$ for each $b$.

A function $g$ can be chosen such that it does not take the unit value on a non-void clopen subset $W$ in $\Lambda$. Indeed, otherwise $g(W)=\{1\}$ and $W$ would be disjoint from $\bigcup_{b<1} \Lambda_{b}$ and the function $g$ could be redefined due to the periodicity of the exponential function. Then a point $y \in W$ would exist so that $g(y) \neq 1$.

For each $\epsilon>0$ a positive number $\delta>0$ exists such that for every partition $\alpha=\left\{b_{0}, \cdots, b_{n}\right\}$ of the segment $[0,1]$ of diameter $|\alpha|<\delta$ and points $c_{j} \in\left[b_{j-1}, b_{j}\right]$ the inequality is satisfied:

5) $\left\|K-\sum_{j} c_{j}\left({ }_{b_{j}} \hat{E}-{ }_{b_{j-1}} \hat{E}\right)\right\|<\epsilon$, consequently,

6) $\lim _{\delta \downarrow 0}\left\|\left.T\right|_{t_{X}}-\left({ }_{b_{j}} \hat{E}-_{b_{j-1}} \hat{E}\right) \sum_{j} \exp \left\{2 \pi M_{t}\left(u_{j}\right) g\left(u_{j}\right)\right\}\right\|=0$, where $u_{j}=u_{j, t} \in \Lambda_{b_{j}}^{t} \backslash \Lambda_{b_{j-1}}^{t}$ and the sum is by $j$ for which the subset $\Lambda_{b_{j}}^{t} \backslash \Lambda_{b_{j-1}}^{t}$ is non-void, since the exponential function on $\Psi_{t}$ is continuous. Certainly coefficients $\exp \left\{2 \pi M_{t}\left(u_{j}\right) g\left(u_{j}\right)\right\}$ are in $f_{t}\left(\Lambda^{t}\right)$ and hence in $\operatorname{sp}\left(\left.T\right|_{t_{X}}\right)$.

Since $v<\infty$ by the conditions of this theorem, the purely imaginary unit sphere $\mathcal{S}_{v}$ is compact in the Cayley-Dickson algebra $\mathcal{A}_{v}$. Then

$s p(T) \subset \operatorname{cl}\left[\bigcup_{t \in Y} s p\left(\left.T\right|_{t_{X}}\right)\right] \subset \mathcal{S}_{v}$, consequently, for each $\delta>0$ a finite $\delta$-net of points in $s p(T)$ exists. Therefore, for each $\epsilon>0$ a finite family of points $u_{j, t_{k}} \in \bigcup_{t \in \Upsilon} \Lambda^{t}$ exists so that

$$
\left\|T-\sum_{j, k}\left({ }_{b_{j}} \hat{E}-_{b_{j-1}} \hat{E}\right) \cdot \exp \left\{2 \pi M_{t}\left(u_{j, t_{k}}\right) g\left(u_{j, t_{k}}\right)\right\}\right\|<\epsilon,
$$

since $\operatorname{cl}\left[\bigcup_{t \in Y} \Psi_{t}\right]=\mathcal{A}_{v}$.

2) In accordance with the conditions of Theorem 9 , an operator $T$ is an $\mathcal{A}_{1}$ meta-invariant either right or left $\mathcal{A}$, linear operator, consequently, $\mathcal{A}$, graded resolution $\left\{{ }_{b} \hat{E}: b \in[0,1]\right\}$ of the identity can be chosen either right or left $\mathcal{A}_{v}$ linear for all $b$ (see Theorem 4). Then $T$ due to its either right or left $\mathcal{A}_{v}$ linearity is completely characterized by its restriction on $X_{0} \oplus X_{1} i_{1} \quad$ (up to continuous with its inverse isomorphism of $\mathcal{A}_{v}$ Hilbert spaces as two-sided $\mathcal{A}_{v}$ modules). Therefore, to the operator $T$ up to an isomorphism of $C^{*}$ algebras a function $f \in C\left(\Lambda, \mathcal{A}_{1}\right)$ corresponds such that $f(x)=\exp \left\{2 \pi i_{1} g(x)\right\}$, where $g(x) \in \boldsymbol{R}$ for each $x \in \Lambda$, $\mathcal{A}_{1}=\boldsymbol{R} \oplus \boldsymbol{R} i_{1}$. Equivalently up to an isomorphism one can take a purely imaginary (constant) number $M$ instead of $i_{1}$, that is $M \in \Psi_{t}, \operatorname{Re}(M)=0,|M|=1$, where $\Psi_{t}$ is isomorphic with $\mathcal{A}_{1}$ for every $t \in \Upsilon$ (see \$2.9). Thus generally $f(x)=\exp \{2 \pi M g(x)\}$. This operator $K$ can be chosen $\mathcal{A}_{1}$ meta-invariant on $X$, since $T$ is $\mathcal{A}_{1}$ meta-invariant on $X$.

In view of (1) we get

7) $\lim _{\delta \downarrow 0}\left\|T-\sum_{j} \exp \left\{2 \pi M g\left(t_{j}\right)\right\}\left({ }_{b_{j}} \hat{E}-{ }_{b_{j-1}} \hat{E}\right)\right\|=0$,

where the operator $K$ is given by Formula (4).

11) Remark. For an infinite dimensional Hilbert space $X$ over the Cayley-Dickson algebra $\mathcal{A}_{v}$ one has that $X \oplus X$ and $X$ are isomorphic as Hilbert two-sided $\mathcal{A}_{v}$ modules, since $X_{0}$ and $X_{0} \oplus X_{0}$ are isomorphic as real Hilbert spaces.

12) Theorem. Let $\Lambda$ be a compact Hausdorff space, let also $X$ be an $\mathcal{A}_{v}$ Hilbert space, and let $\phi$ be a representation of $C\left(\Lambda, \Psi_{t}\right)$ on ${ }^{t} X$ for each $t \in \Upsilon$, where $2 \leq v, \Psi_{t}$ is isomorphic with $\mathcal{A}_{r}$ for each $t \in \Upsilon, \quad 2 \leq r \leq \min (3, v)$ so that Conditions 2.9

(M1-M3) are fulfilled. Then to each Borel subset $G$ of $\Lambda$ a graded projection $\hat{\boldsymbol{E}}(G)$ corresponds such that

1) $\hat{\boldsymbol{E}}(G) \in \boldsymbol{A}$, where $\boldsymbol{A}$ denotes the strong operator closure of $\operatorname{span}_{R} \bigcup_{t \in \Gamma} \phi C\left(\Lambda, \Psi_{t}\right)$;

2) $\hat{\boldsymbol{E}}(G)=\Lambda\{\hat{\boldsymbol{E}}(U): G \subset U ; U$ is open $\}$;

3) $\hat{\boldsymbol{E}}\left(\bigcup_{n=1}^{\infty} G_{n}\right) \sum_{n=1}^{\infty} \hat{\boldsymbol{E}}\left(G_{n}\right)$ for each countable family $\left\{G_{n}: n\right\}$ of mutually disjoint Borel subsets of $\Lambda$; also $\hat{\boldsymbol{E}}\left(G_{n}\right) \hat{\boldsymbol{E}}\left(G_{k}\right)=0$ for each $n \neq k, \quad \hat{\boldsymbol{E}}(\varnothing)=0$;

4) If the $\mathcal{A}_{v}$ span of ranges of those $\phi(f)$ such that $f \in C\left(\Lambda, \mathcal{A}_{v}\right)$ and $f$ vanishes on $\Lambda \backslash B_{0}$ is dense in $X$ for $B_{0}$ a Borel subset of $\Lambda$, then $E\left(B_{0}\right)=I$;

5) The mapping $B(\Lambda) \ni G \mapsto<\hat{\boldsymbol{E}}(G) x, x>=: \hat{\mu}(G ; x, x)$ is a regular Borel measure so that

$$
\langle\phi(f) x, x\rangle=\int_{\Lambda}\langle\hat{\boldsymbol{E}}(\mathrm{d} y) \cdot f(y) x, x\rangle
$$


for each continuous function $f \in C\left(\Lambda, \mathcal{A}_{v}\right)$, where $B(\Lambda)$ denotes a Borel $\sigma$-algebra of all Borel subsets in $\Lambda$.

Proof. A multiplication $\left[C\left(\Lambda, \Psi_{t}\right)\right]^{2} \ni(f, g) \mapsto f g \in C\left(\Lambda, \Psi_{t}\right)$ and an involution $C\left(\Lambda, \Psi_{t}\right)$ э $f \mapsto f^{*} \in C\left(\Lambda, \Psi_{t}\right)$ are defined pointwise $(f g)(y)=f(y) g(y)$ and $f^{*}(y)=\overline{[f(y)]}$ for each $y \in \Lambda$. The $C^{*}$-algebra $C\left(\Lambda, \mathcal{A}_{v}\right)$ is quasi-commutative, consequently, $\boldsymbol{A}$ is a quasi-commutative $C^{*}$ algebra.

Suppose that $U$ is an open subset in $\Lambda$ and a continuous function $f: \Lambda \rightarrow[0,1]$ is given such that $f(\Lambda \backslash U)=\{0\}$. Then $0 \leq \phi(f) \leq I$. Denote by $\mathcal{F}(U)$ the family of all such functions $f$ directed by the natural order of functions: $f \leq g$ if $f(y) \leq g(y)$ for each $y \in \Lambda$. Therefore, the family $\phi(\mathcal{F}(U))$ has a supremum $h_{U}$ in the quasi-commutative $C^{*}$-algebra $\boldsymbol{A}$ over $\mathcal{A}_{v}$. This element $h_{U}$ is a projection, since $\left\{f^{2}: f \in F(U)\right\}=F(U)$. For a Borel subset $G$ of $\Lambda$ we put $\hat{\boldsymbol{E}}(G)=\Lambda\left\{h_{U}: G \subset U, U\right.$ is open in $\left.\Lambda\right\}$.

If $x \in{ }^{t} X$ is a unit vector $\|x\|=1$, then

$f \mapsto\langle\phi(f) x, x\rangle=: p_{x, x}(f)$ is a state of $C\left(\Lambda, \mathcal{A}_{v}\right)$. Indeed, $p_{x, x}$ on $C\left(\Lambda, \mathcal{A}_{v}\right)$ is $\boldsymbol{R}$-linear and $\mathcal{A}_{v}$ additive, $p_{x, x}: C(\Lambda, \boldsymbol{R}) \rightarrow \boldsymbol{R}, p_{x, x}(f) \geq 0$ for each nonnegative function on $\Lambda$, moreover, $p_{x, x}(I)=\langle x, x\rangle=1$ and for every non-negative continuous function $g$ on $\Lambda$ :

6) $p_{x, x}(b g)=\sum_{j, k}\left\langle b \phi(g) x_{j} i_{j}, x_{k} i_{k}\right\rangle=b\langle\phi(g) x, x\rangle$,

particularly for $g=1$ also:

$$
p_{x, x}(b I)=b\langle x, x\rangle=b
$$

for each $b \in \mathcal{A}$, due to the identity $\left(a i_{j}\right) i_{k}^{*}+\left(a i_{k}\right) i_{j}^{*}=a 2 \operatorname{Re}\left(i_{j} i_{k}^{*}\right)$ for each $a \in \mathcal{A}_{v}$ and $j, k=0,1,2, \cdots$ (see also Formula 4.2 (7) in [13] and $\S \S 2.8$ ), since a continuous image of a compact topological space is compact by Theorem 3.1.10 [14] and hence each continuous function $f: \Lambda \rightarrow \mathcal{A}_{v}$ on a compact topological space $\Lambda$ is bounded. From the Riesz representation theorem for continuous $\boldsymbol{R}$-linear non-negative functionals on the $\boldsymbol{R}$-linear space $C(\Lambda, \boldsymbol{R})$ we get that

7) $\langle\phi(f) x, x\rangle=\int_{\Lambda} f(y) \hat{\mu}(\mathrm{d} y ; x, x)$

for each $f \in C(\Lambda, \boldsymbol{R})$, where $\hat{\mu}(* ; x, x)$ is a regular Borel $\sigma$-additive non-negative measure on $\Lambda$.

Then Formulas $(6,7)$ generate $\hat{\mu}(\mathrm{d} y ; x, x)$ on the space $C\left(\Lambda, \mathcal{A}_{v}\right)$ (see $\left.\$ 2.23[12]\right)$. The family $\hat{\mu}(\mathrm{d} y ; x, x)$ generate the family $\hat{\mu}(\mathrm{d} y ; x, y)$ due to the bi- $\mathcal{A}_{v}$ additivity

$$
\begin{aligned}
\hat{\mu}(\mathrm{d} y ; x \pm z, x \pm z)= & \hat{\mu}(\mathrm{d} y ; x, x) \pm \hat{\mu}(\mathrm{d} y ; x, z) \\
& \pm \hat{\mu}(\mathrm{d} y ; z, x)+\hat{\mu}(\mathrm{d} y ; z, z)
\end{aligned}
$$

for all $x, z \in \operatorname{span}_{\boldsymbol{R}} \bigcup_{t \in \Upsilon}{ }^{t} X \quad$ and bi- $\boldsymbol{R}$-linearity

$$
\hat{\mu}(\mathrm{d} y ; \alpha x, x)=\hat{\mu}(\mathrm{d} y ; x, \alpha x)=\alpha \hat{\mu}(\mathrm{d} y ; x, x)
$$

for each $\alpha \in \boldsymbol{R}$ provided by the corresponding properties of $p_{x, x}$. This induces $\hat{\mu}(\mathrm{d} y ; x, y)$ and hence $\hat{\boldsymbol{E}}(G)$ on $\mathcal{B}(\Lambda) \times X$ due to Conditions $2.9(M 1-M 3)$.

The measure $\hat{\mu}(\mathrm{d} y ; x, x)$ is inner regular, that is for each $\epsilon>0$ and an open subset $U$ in $\Lambda$ a compact subset $B$ in $U$ exists such that $\hat{\mu}(U \backslash B ; x, x)<\epsilon$. The topological space $\Lambda$ is normal, consequently, for each closed subset $B$ in an open subset $U$ in $\Lambda$ a continuous function $f: \Lambda \rightarrow[0,1]$ exists so that $f(B)=\{1\}$ and $f(\Lambda \backslash U)=\{0\}$. For such function we have

$$
\begin{aligned}
\hat{\mu}(B ; x, x) & \leq \int_{\Lambda} f(y) \hat{\mu}(\mathrm{d} y ; x, x) \\
& =\langle\phi(f) x, x\rangle \leq\langle\hat{\boldsymbol{E}}(U) x, x\rangle,
\end{aligned}
$$

consequently, $\hat{\mu}(U ; x, x) \leq\langle\hat{\boldsymbol{E}}(U) x, x\rangle$, since $f$ is arbitrary with such properties. In accordance with the definition of $\hat{\boldsymbol{E}}$ we have $\langle\hat{\boldsymbol{E}}(U) x, x\rangle \leq \hat{\mu}(U ; x, x)$, hence $\hat{\mu}(U ; x, x)=\langle\hat{\boldsymbol{E}}(U) x, x\rangle$. The outer regularity of $\hat{\mu}(\mathrm{d} y ; x, x)$ implies that

$$
\begin{aligned}
\hat{\mu}(W ; x, x) & =\inf \{\hat{\mu}(U ; x, x): W \subset U, U \text { is open }\} \\
& =\inf \{\langle\hat{\boldsymbol{E}}(U) x, x\rangle: W \subset U, U \text { is open }\} \\
& =\langle\hat{\boldsymbol{E}}(W) x, x\rangle
\end{aligned}
$$

for each Borel subset $W$ in $\Lambda$. For a disjoint sequence of Borel subset $G_{n}$ in $\Lambda$ we get

$$
\begin{aligned}
\left\langle\hat{\boldsymbol{E}}\left(\bigcup_{n=1}^{\infty} G_{n}\right) x, x\right\rangle & =\hat{\mu}\left(\bigcup_{n=1}^{\infty} G_{n} ; x, x\right)=\sum_{n=1}^{\infty} \hat{\mu}\left(G_{n} ; x, x\right) \\
& =\sum_{n=1}^{\infty}\left\langle\hat{\boldsymbol{E}}\left(G_{n}\right) x, x\right\rangle .
\end{aligned}
$$

If $x \in \hat{\boldsymbol{E}}\left(G_{m}\right)$ for some $m$ and $\|x\|=1$, then

$$
1 \geq \sum_{n=1}^{\infty}\left\langle\hat{\boldsymbol{E}}\left(G_{n}\right) x, x\right\rangle \geq\left\langle\hat{\boldsymbol{E}}\left(G_{m}\right) x, x\right\rangle=1 .
$$

This implies that $\left\langle\hat{\boldsymbol{E}}\left(G_{n}\right) x, x\right\rangle=0$ for each $n \neq m$, since $\left\langle\hat{\boldsymbol{E}}\left(G_{n}\right) x, x\right\rangle \geq 0$ for each $n$. Thus

$$
\begin{aligned}
E\left(G_{n}\right) E\left(G_{m}\right)= & 0 \text { for each } n \neq m, \text { consequently, } \\
& \hat{\boldsymbol{E}}\left(\bigcup_{n=1}^{\infty} G_{n}\right)=\sum_{n=1}^{\infty} \hat{\boldsymbol{E}}\left(G_{n}\right) .
\end{aligned}
$$

If a set $B_{0}$ is as in (4) and $U$ is an open subset containing $B_{0}$ and $f \in C(\Lambda, \boldsymbol{R})$ with $f(\Lambda \backslash U)=\{0\}$, a function $f$ is non-zero identically, then a range graded projection of $\phi(g)$ is a graded sub-projection of $\hat{\boldsymbol{E}}(U)$, where $g=f /\|f\|$. On the other hand, the range graded projection of $\phi(g)$ is the same as that of $\phi(f)$, since $\|f\|>0$ and $\phi(|f|)=\phi\left(f^{+}\right)+\phi\left(f^{-}\right)$and each operator in $L_{q}(X)$ is $\boldsymbol{R}$-linear, where 
$f^{+}(y)=\max (0, f(y))$ and $f^{-}(y)=-\min (0, f(y))$ for each $y \in \Lambda$. Thus $\hat{\boldsymbol{E}}(U)$ contains the range graded projection of the image of each function $f \in C\left(\Lambda, \mathcal{A}_{v}\right)$ so that $f$ vanishes on $\Lambda \backslash B_{0}$, since the $\mathcal{A}_{v}$ span of functions from $C(\Lambda, \boldsymbol{R})$ is everywhere dense in

$$
C\left(\Lambda, \mathcal{A}_{v}\right)=C(\Lambda, \boldsymbol{R}) i_{0} \oplus \cdots \oplus C(\Lambda, \boldsymbol{R}) i_{m} \oplus \cdots .
$$

Hence $\hat{\boldsymbol{E}}\left(B_{0}\right)=I$ due to Property (2).

13) Corollary. Suppose that $\phi$ is an essential representation of $C_{0}\left(R, \mathcal{A}_{v}\right), 2 \leq v$, (see $\S 6$ and Theorem 12), then a possibly unbounded $\mathcal{A}_{v}$ graded resolution of the identity $\left\{{ }_{b} \hat{E}: b \in \boldsymbol{R}\right\}$ exists such that

$$
\text { 1) }\langle\phi(f) x, x\rangle=\int_{-\infty}^{\infty} \mathrm{d}\left\langle{ }_{t} \hat{E} \cdot f(t) x, x\right\rangle
$$

for each $f \in C_{0}\left(\boldsymbol{R}, \mathcal{A}_{v}\right)$.

Proof. Consider a one-point (Alexandroff) compactification $\alpha \boldsymbol{R}=\boldsymbol{R} \bigcup\{\infty\}$ of the real field. Then

$C_{0}\left(\boldsymbol{R}, \mathcal{A}_{v}\right)$ is isomorphic with

$C_{0}\left(\alpha \boldsymbol{R}, \mathcal{A}_{v}\right)=\left\{f \in C\left(\alpha \boldsymbol{R}, \mathcal{A}_{v}\right): f(\infty)=0\right\} \quad$ (see $\left.\S 6\right)$. The latter is a quasi-commutative subalgebra in $C\left(\alpha \boldsymbol{R}, \mathcal{A}_{v}\right)$.

In accordance with Theorem 12 there exists a graded projection valued measure on $\alpha R$ such that

$$
\langle\phi(f) x, x\rangle=\int_{\boldsymbol{R}} \hat{\mu}(\mathrm{d} y ; x, x) \cdot f(y)
$$

for each $f \in C_{0}(\boldsymbol{R}, \boldsymbol{R})$, moreover, $\hat{\mu}(\boldsymbol{A} ; x, x)=\langle\hat{\boldsymbol{E}}(\boldsymbol{A}) x, x\rangle$ for each Borel subset $\boldsymbol{A}$ in $\alpha \boldsymbol{R}$. Put ${ }_{b} \hat{E}=I-\hat{\boldsymbol{E}}(b, \infty)$, then ${ }_{b} \hat{E}$ is a graded resolution of the identity (possibly unbounded). Indeed, $\hat{\boldsymbol{E}}(b, \infty) \leq \hat{\boldsymbol{E}}(t, \infty)$ for each $t \leq b$, consequently,

${ }_{t} \hat{E} \leq{ }_{b} E$. Statement (4) of Theorem 12 implies that $\hat{\boldsymbol{E}}(\boldsymbol{R})=I$.

Then we have $\hat{\boldsymbol{E}}(k, \infty)=\sum_{n=k}^{\infty} \hat{\boldsymbol{E}}(n, n+1]$ for each integer $k$ and $\Lambda_{k} \hat{E}(k, \infty)=0$. Therefore,

$$
I=I-\Lambda_{b} \hat{\boldsymbol{E}}(b, \infty)=\vee_{b}[I-\hat{\boldsymbol{E}}(b, \infty)]=\vee_{b b} \hat{E} .
$$

On the other hand, one has

$$
I=\hat{E}(\boldsymbol{R})=\sum_{n=-\infty}^{\infty} \hat{\boldsymbol{E}}(n, n+1]=\bigvee_{n=-\infty}^{\infty} \hat{\boldsymbol{E}}(n, \infty),
$$

consequently,

$$
0=I-\bigvee_{n=-\infty}^{\infty} \hat{\boldsymbol{E}}(n, \infty)=\wedge_{n=-\infty}^{\infty}(I-\hat{\boldsymbol{E}}(n, \infty))=\wedge_{n=-\infty n}^{\infty} \hat{E}
$$

and

$$
0 \leq \wedge_{b b} \hat{E} \leq \wedge_{n=-\infty n}^{\infty} \hat{E}=0 .
$$

Consider now the sequence $b(n)=b+\frac{1}{n}$ for $n \in N$. Then

$$
\hat{\boldsymbol{E}}(b, \infty)=\hat{\boldsymbol{E}}(b+1, \infty)+\sum_{n=1}^{\infty} \hat{\boldsymbol{E}}(b(n+1), b(n)]
$$

such that

$$
\begin{aligned}
\wedge_{n=1 b(n)}^{\infty} \hat{E} & \leq{ }_{b+1} \hat{E} \wedge\left\{\wedge_{n=1}^{\infty}(I-E(b(n+1), b(n)])\right\} \\
& =I-E(b, \infty)={ }_{b} \hat{E} .
\end{aligned}
$$

Then we get ${ }_{b} \hat{E}=\wedge_{b<t} \hat{E}$ and $\left\{{ }_{b} \hat{E}: b\right\}$ is a graded resolution of the identity, since

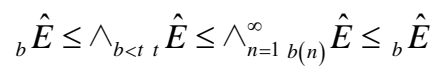

and $\hat{\boldsymbol{E}}$ is the graded projection valued measure.

Certainly one has the identities

$$
\hat{\boldsymbol{E}}(b, t]=\hat{\boldsymbol{E}}(b, \infty)-\hat{\boldsymbol{E}}(t, \infty)={ }_{t} \hat{E}-{ }_{b} \hat{E},
$$

also $\hat{\mu}((b, t] ; x, x)=\left\langle{ }_{t} \hat{E} x, x\right\rangle-\left\langle{ }_{b} \hat{E} x, x\right\rangle$. Together with Statement (5) of Theorem 12 this means that

$$
\text { 2) } \begin{aligned}
\langle\phi(f) x, x\rangle & =\int_{R} \hat{\mu}(\mathrm{d} t ; x, x) \cdot f(t) \\
& =\int_{-\infty}^{\infty} \mathrm{d}\left\langle{ }_{t} \hat{E} \cdot f(t) x, x\right\rangle
\end{aligned}
$$

for each $f \in C_{0}\left(\boldsymbol{R}, \mathcal{A}_{v}\right)$ due to the $\mathcal{A}_{v}$ gradation of $\hat{\boldsymbol{E}}$.

14) Remark. If $T$ is a bounded normal $\mathcal{A}_{r}$ metainvariant operator on an $\mathcal{A}_{v}$ Hilbert space $X$ and $\boldsymbol{A}$ is a quasi-commutative $C^{*}$-algebra over the CayleyDickson algebra $\mathcal{A}_{v}$ generated by $T$ and $T^{*}$ and $I, 2 \leq v, 1 \leq r \leq \min (3, v)$, then in accordance with Theorem 4 and Lemma 3 there exists an $\mathcal{A}_{v}$ graded projection valued measure $\hat{\boldsymbol{E}}$ on either the one-point compactification $\Phi=\alpha \boldsymbol{R}$ of $\boldsymbol{R}$ for a self-adjoint operator $T$ or on the one-point compactification $\Phi=\mathcal{A}_{v}$ of $\mathcal{A}_{v}$ for a normal operator $T$ such that

$$
\text { 1) }\langle f(T) x, x\rangle=\int_{\Phi} \hat{\mu}(\mathrm{d} b ; x, x) \cdot f(b)
$$

for each $f \in C\left(\Phi, \mathcal{A}_{v}\right)$ and $x \in X$, where $\hat{\mu}(B ; x, x)=\langle E(B) x, x\rangle$ for every Borel subset $B$ in $\Phi$. A spectrum $\Lambda=s p(T)$ is embedded into $\Phi$. If $U$ is an open subset of $\Phi$ disjoint from $s p(T)$ and $f \in \mathcal{F}(U)$ (see $\S 12$ ), then $f(T)=0$. Therefore, $\hat{\boldsymbol{E}}(U)=0$ and we write

$$
\text { 2) }\langle f(T) x, x\rangle=\int_{s p(T)} \hat{\mu}(\mathrm{d} b ; x, x) \cdot f(b)
$$

and $\hat{\boldsymbol{E}}$ is the spectral measure of $T$ on the Borel $\sigma$ algebra $\mathcal{B}(s p(T))$. Then for a self-adjoint operator $T$, one gets that ${ }_{b} \hat{E}$ is a bounded graded resolution of the identity such that ${ }_{b} \hat{E}=0$ for $b<-\|T\|$ and ${ }_{b} \hat{E}=I$ for $b \geq\|T\|$, consequently,

$$
\text { 3) }\langle f(T) x, x\rangle=\int_{-\|T\|}^{\|T\|} \mathrm{d}\left\langle{ }_{b} \hat{E} f(b) x, x\right\rangle
$$

for each vector $x \in X$ (see Corollary 13). Using the polarization as in $\$ 14$ we get 
4) $\langle f(T) x, y\rangle=\int_{-\|T\|}^{\|T\|} \mathrm{d}\left\langle{ }_{b} \hat{E} \cdot f(b) x, y\right\rangle$

for each $x, y \in X$.

Formula (1) extends by continuity of the functional on the $\mathcal{A}_{v}$ vector space $\boldsymbol{B}\left(\Phi, \mathcal{A}_{v}\right)$ of all bounded Borel measurable functions $f: \Phi \rightarrow \mathcal{A}_{v}$, since

$$
\left|\int_{\Phi} \mathrm{d}\langle\hat{\boldsymbol{E}}(\mathrm{d} b) \cdot f(b) x, y\rangle\right| \leq\|x\|\|y\| \sup _{b \in \Phi}|f(b)| .
$$

For a normal bounded operator $T$ such polarization gives:

$$
\text { 5) }\langle f(T) x, y\rangle=\int_{\Phi} \hat{\mu}(\mathrm{d} b ; x, y) \cdot f(b)
$$

for every $x, y \in X$ and each $f \in \boldsymbol{B}\left(\Phi, \mathcal{A}_{v}\right)$. Then the identities are satisfied:

6) $\langle\tilde{f}(T) x, x\rangle$

$$
\begin{aligned}
& =\int_{\Phi}\langle\hat{\boldsymbol{E}}(\mathrm{d} b) \cdot \tilde{f}(b) x, x\rangle=\int_{\Phi}\left\langle x \cdot f(b) \hat{\boldsymbol{E}}^{*}(\mathrm{~d} b) x\right\rangle \\
& =\int_{\Phi}\langle\hat{\boldsymbol{E}} \overline{(\mathrm{d} b) \cdot f(b)} x, x\rangle=\langle\overline{f(T) x, x}\rangle \\
& =\left\langle[f(T)]^{*} x, x\right\rangle
\end{aligned}
$$

for each $x \in X$, consequently,

7) $\tilde{f}(T)=[f(T)]^{*}$

for every $f \in \boldsymbol{B}\left(\Phi, \mathcal{A}_{v}\right)$. If $f, g \in \boldsymbol{B}\left(\Phi, \mathcal{A}_{v}\right)$, then one certainly obtains

$$
\text { 8) } \begin{aligned}
& \langle(c f+g)(T) x, y\rangle \\
= & \int_{\Phi}\langle\hat{\boldsymbol{E}}(\mathrm{d} b) \cdot(c f(b)+g(b)) x, y\rangle \\
= & c \int_{\Phi}\langle\hat{\boldsymbol{E}}(\mathrm{d} b) \cdot f(b) x, y\rangle+\int_{\Phi}\langle\hat{\boldsymbol{E}}(\mathrm{d} b) \cdot g(b) x, y\rangle \\
= & c\langle f(T) x, y\rangle+\langle g(T) x, y\rangle
\end{aligned}
$$

for all $x, y \in X$, consequently,

$$
\text { 9) }(c f+g(T))=c f(T)+g(T)
$$

for each real number $c \in \boldsymbol{R}$. Particularly, for the characteristic function $\chi_{B}$ of a Borel subset contained in $\Phi$ we get the equalities:

$$
\left\langle\chi_{B}(T) x, x\right\rangle=\int_{\Phi}\left\langle\hat{\boldsymbol{E}}(\mathrm{d} b) \cdot \chi_{B}(b) x, x\right\rangle=\langle\hat{\boldsymbol{E}}(B) x, x\rangle,
$$

consequently,

10) $\chi_{B}(T)=\hat{\boldsymbol{E}}(B)$ and $\chi^{2}(T)=\chi(T)$.

If a Borel subset $C$ is disjoint form $B$, then $0=\chi_{B} \chi_{C}$, hence $\left(\chi_{B}(T) \pm \chi_{C}(T)\right)^{2}=\left(\chi_{B} \pm \chi_{C}\right)^{2}(T)$. Recall that a step function by the definition is a finite $\mathcal{A}_{v}$ vector combination of characteristic functions. For any two step Functions $g=\sum_{l=1}^{m} a_{l} \chi_{A_{l}}$ and $h=\sum_{k=1}^{n} b_{k} \chi_{B_{k}} \quad$ with real coefficients $a_{l}$ and $b_{k}$ and mutually disjoint sets $A_{l} \cap A_{k}=\varnothing$ and $B_{l} \cap B_{k}=\varnothing$ for each $k \neq l$ this implies the identities

$$
g^{2}(T)=\sum_{l=1}^{m} a_{l}^{2} \chi_{A_{l}}(T)=\sum_{l=1}^{m} a_{l}^{2} \chi_{A_{l}}^{2}(T)=[g(T)]^{2}
$$

and

$$
\begin{aligned}
& \left(\sum_{l=1}^{m} a_{l} \chi_{A_{l}}(T) \pm \sum_{k=1}^{n} b_{k} \chi_{B_{k}}(T)\right)^{2} \\
& =\sum_{l=1}^{m} a_{l}^{2} \chi_{A_{l}}(T)+\sum_{k=1}^{n} b_{k}^{2} \chi_{B_{k}}(T) \pm \sum_{l, k}\left[a_{l} b_{k}+b_{k} a_{l}\right] \chi_{A_{l} \cap B_{k}}(T) \\
& =g^{2}(T)+h^{2}(T) \pm g(T) h(T) \pm h(T) g(T),
\end{aligned}
$$

consequently, $g(T) h(T)=(g h)(T)$. Each bounded Borel function is a limit of step functions, where a limit is taken relative to the family of all semi-norms

$$
\text { 11) }\|g\|_{\hat{E} ; x}:=\int_{\Phi}|\langle\hat{\boldsymbol{E}}(\mathrm{d} b) \cdot g(b) x, x\rangle| \text {, }
$$

$x \in X,\|x\|=1$. Therefore, for each bounded real-valued Borel functions $g, h \in \boldsymbol{B}(\Phi, \boldsymbol{R})$ the formula

12) $(g h)(T)=g(T) h(T)$

is valid. But then $g(b) \tilde{g}(b)=|g(b)|^{2}$ for each $b \in \Phi$ and $g(T) \tilde{g}(T)=(g \tilde{g})(T)$, consequently,

$$
[(g \pm h)(\tilde{g} \pm \tilde{h})](T)=(g(T) \pm h(T))(\tilde{g}(T) \pm \tilde{h}(T))
$$

and hence

$$
\begin{aligned}
2[\operatorname{Re}(g \tilde{h})](T) & =[g \tilde{h}+h \tilde{g}](T) \\
& =g(T) \tilde{h}(T)+h(T) \tilde{g}(T)
\end{aligned}
$$

for each $g, h \in B\left(\Phi, \mathcal{A}_{v}\right)$. If $g$ and $h$ are polynomials in variables $z, z^{*} \in \mathcal{A}_{v}$ this implies

13) $(g \circ \tilde{h})(T)+(h \circ \tilde{g})(T)=g(\tilde{h}(T))+h(\tilde{g}(T))$.

In view of the Stone-Weierstrass theorem the set of $\mathcal{A}_{v}$-valued polynomials on the ball

$$
B\left(\mathcal{A}_{v}, 0, w\right):=\left\{z \in \mathcal{A}_{v}:\|z\| \leq w\right\}
$$

is dense in $C\left(B\left(\mathcal{A}_{v}, 0, w\right), \mathcal{A}_{v}\right)$. Thus Formula (13) is spread on $C\left(\Phi, \mathcal{A}_{v}\right)$, since $s p(T) \subset B\left(\mathcal{A}_{v}, 0, w\right)$ for $w \geq\|T\|$ and due to Formula (2). Together with (12) this induces the formula.

$$
\text { 14) }(g \circ h)(T)=g(h(T))
$$

for each real-valued continuous function $g \in C(\Phi, \boldsymbol{R})$ 
and every bounded real-valued Borel function $h \in \boldsymbol{B}(\Phi, \boldsymbol{R})$, since $h(T)$ is a self-adjoint operator (see also 9 ). In the latter situation

$$
g(h(T))=\tilde{g}(h(T))=[g(h(T))]^{*}
$$

and if $g \geq 0$, then $g(h(T)) \geq 0$. Therefore, for any increasing sequence of continuous real-valued functions $g_{n} \in C(\Phi, \boldsymbol{R})$ a sequence $\left\{g_{n}(h(T)): n\right\}$ is increasing and composed of self-adjoint operators. If in addition $g_{n}$ converges point-wise to a bounded Borel function $g$, then $g_{n} \circ h$ converges point-wise to $g \circ h$.

There is said that the mapping

$$
\boldsymbol{B}(\Phi, \boldsymbol{R}) \text { э } f \mapsto f(T) \in L_{q}(X)
$$

with the monotone sequential convergence property is $\sigma$-normal.

15) Theorem. Suppose that $T$ is a bounded normal $\mathcal{A}_{r}$ meta-invariant operator on an $\mathcal{A}_{v}$ Hilbert space $X$ and $\boldsymbol{A}$ is a quasi-commutative $C^{*}$-algebra over the Cayley-Dickson algebra $\mathcal{A}_{v}$ generated by $T$ and $T^{*}$ and $I, 2 \leq v, 1 \leq r \leq \min (3, v), \Phi=\mathcal{A}_{v}$ with $v \leq \aleph_{0}$. Then the-homomorphism $C\left(\Phi, \mathcal{A}_{v}\right)$ э $f \mapsto f(T) \in \boldsymbol{A}$ extends to a $\sigma$-normal-homomorphism

$\boldsymbol{B}\left(\Phi, \mathcal{A}_{v}\right)$ э $f \mapsto f(T) \in \boldsymbol{C}$, where $\boldsymbol{C}$ denotes a quasicommutative von Neumann algebra over $\mathcal{A}_{v}$ consisting of operators quasi-commuting with each operator quasicommuting with $\boldsymbol{A}$ such that

1) $\|f(T)\| \leq \sup \{|f(b)|: b \in \operatorname{sp}(T)\}$ and

2) $\langle f(T) x, x\rangle=\int_{\Phi}\langle\hat{\boldsymbol{E}}(\mathrm{d} b) \cdot f(b) x, x\rangle$

$$
=\int_{s p(T)} \hat{\mu}(\mathrm{d} b ; x, x) \cdot f(b),
$$

where $\hat{\mu}(\mathrm{d} b ; x, x) \cdot f(b)=\langle\hat{\boldsymbol{E}}(\mathrm{d} b) \cdot f(b) x, x\rangle$ for each $x \in X$. If $g \in \boldsymbol{B}\left(\Phi, \mathcal{A}_{v}\right)$, then $\tilde{g}(T)=[g(T)]^{*}$, moreover, if $\left.g\right|_{s p(T)}=0$, then $g(T)=0$. For each

$g, h \in \boldsymbol{B}(\Phi, \boldsymbol{R})$ the composition is $(g \circ h)(T)=g(h(T))$. The mapping $\mathcal{B}(\Phi)$ э $G \mapsto \chi_{G}(T)=\hat{\boldsymbol{E}}(G)$ is an $\mathcal{A}_{v}$ graded projection valued measure. If an operator $T$ is self-adjoint, then it possesses an $\mathcal{A}_{v}$ graded spectral resolution of the identity $\left\{{ }_{b} \hat{E}: b\right\}$ with ${ }_{b} \hat{E}=I-\hat{\boldsymbol{E}}(b, \infty)$ and $\Phi=\alpha \boldsymbol{R}$, where $2 \leq v$.

Proof. Part of the proof is given in Section 16.

In view of Theorem V.4.4 [17] a point-wise $\lim f=\lim _{n} f_{n}$ of a sequence of $(\mathcal{M}, \mathcal{B}(\boldsymbol{R}))$-measurable functions $f_{n}: V \rightarrow \boldsymbol{R}$ converging at each point $b \in V$ of a set $V$ with a $\sigma$-algebra $\mathcal{M}$ of its subsets is an $(\mathcal{M}, \mathcal{B}(\boldsymbol{R}))$-measurable function $f$ on $V$, that is $f^{-1}(\mathcal{B}(\boldsymbol{R})) \subset \mathcal{M}$, where $\mathcal{B}(\boldsymbol{R})$ denotes the Borel $\sigma$ algebra of the real field $\boldsymbol{R}$.

Take any vector $x \in X$ and an increasing sequence $f_{n} \in \boldsymbol{B}(\Phi, \boldsymbol{R})$ tending point-wise to a bounded Borel function $f$. Then

$$
\text { 3) } \begin{aligned}
\lim _{n}\left\langle f_{n}(T) x, x\right\rangle & =\lim _{n} \int_{\Phi}\left\langle\hat{\boldsymbol{E}}(\mathrm{d} b) \cdot f_{n}(b) x, x\right\rangle \\
& =\lim _{n} \int_{\Phi}\langle\hat{\boldsymbol{E}}(\mathrm{d} b) \cdot f(b) x, x\rangle \\
& =\langle f(T) x, x\rangle
\end{aligned}
$$

from the monotone convergence theorem. By the decomposition properties of the $\mathcal{A}_{v}$ valued scalar product and the $\mathcal{A}_{v}$ gradation of $\hat{\boldsymbol{E}}$ we get this property on $\boldsymbol{B}\left(\Phi, \mathcal{A}_{v}\right)$, since it is accomplished on $\boldsymbol{B}(\Phi, \boldsymbol{R})$ and each $f \in \boldsymbol{B}\left(\Phi, \mathcal{A}_{v}\right)$ has the form

4) $f=\sum_{j} f_{j} i_{j}$ with real-valued functions $f_{j}$.

Let $U$ be an open subset in $\mathcal{A}_{v}$. It can be written as a countable union of bounded open subsets $V_{k}$ in $\mathcal{A}_{v}$ of radius $R_{k}=\frac{\sup _{x, y \in V_{k}}|x-y|}{2}$, since the topological space $\mathcal{A}_{v}$ is of countable topological weight when $v \leq \aleph_{0}$. The topological space $\mathcal{A}_{v}$ is normal, consequently, there exist open subsets $V_{k, n}$ of radius $\left(1-\frac{1}{n}\right) R_{k}$ contained in $V_{k}$ for which continuous functions $f_{k, n}: \mathcal{A}_{v} \rightarrow[0,1]$ exist such that $f_{k, n}\left(V_{k, n}\right)=\{1\}$ and $f_{k, n}\left(\mathcal{A}_{v} \backslash V_{k}\right)=\{0\}$ such that $\operatorname{cl}\left(V_{k, n}\right) \subset V_{k, n+1}$ for each $n \in N$ and $\bigcup_{n} V_{k, n}=V_{k}$ for every $k$. Take their combination $g_{n}=\vee_{k=1}^{n} f_{k, n}:=\max _{k=1}^{n} f_{k, n}$. From the construction of this sequence we get $\lim _{n} g_{n}(x)=\chi_{U}(x)$ for each $x \in \mathcal{A}_{v}$, consequently, $\lim _{n}\left(1-g_{n}\right)(x)=\chi_{\mathcal{A}_{v} \backslash U}(x)$ for each $x \in \mathcal{A}_{v}$.

Denote by $\mathcal{G}(\Phi)$ the family of all Borel subsets of $\Phi$ whose characteristic function satisfies Equation 16(14). This family contains characteristic functions $g=\chi_{B}$ of each closed and every open subset $B$ of $\Phi$. The $\sigma$-normality property (see Formula (3) ) implies that $\mathcal{G}(\Phi)$ is a $\sigma$-algebra, consequently, $\mathcal{G}(\Phi)$ contains the Borel $\sigma$-algebra $\mathcal{B}(\Phi)$. Thus Formula $16(14)$ is valid for a characteristic function $g=\chi_{B}$ for each Borel subset $B$ of $\Phi$. Using norm limits one gets this formula for any bounded real-valued Borel functions $g$ and $h$.

Mention that the decompositions

5) $\boldsymbol{A}=\boldsymbol{A}_{0} i_{0} \oplus \cdots \oplus \boldsymbol{A}_{m} i_{m} \oplus \cdots$ and

6) $\boldsymbol{C}=\boldsymbol{C}_{0} i_{0} \oplus \cdots \oplus \boldsymbol{C}_{m} i_{m} \oplus \cdots$

Are fulfilled so that the-homomorphism $\theta: C(\Phi, \boldsymbol{R})$ э $f \mapsto f(T) \in \boldsymbol{A}_{0}$ extends to a $\sigma$-normalhomomorphism $\theta: \boldsymbol{B}(\Phi, \boldsymbol{R})$ э $f \mapsto f(T) \in C_{0}$, where $C_{0}$ denotes a commutative von Neumann algebra over $\boldsymbol{R}$ consisting of operators commuting with each operator 
commuting with $\boldsymbol{A}_{0}$. Algebras $C_{k}$ are isomorphic with $C_{0}$ and each algebra $\boldsymbol{A}_{l}$ is isomorphic with $\boldsymbol{A}_{0}$. Then $\theta: \boldsymbol{B}\left(\Phi, \Psi_{t}\right) \rightarrow{ }^{t} \boldsymbol{C}$ is provided for each $t \in \Upsilon$ (see Conditions $2.9(M 1-M 4)$ ), where $C^{*}$-algebras $C\left(\Phi, \mathcal{A}_{v}\right)$ and $\boldsymbol{B}\left(\Phi, \mathcal{A}_{v}\right)$ are considered relative to the point-wise addition and multiplication of functions from the left and right on Cayley-Dickson numbers and point-wise conjugation as involution and with the norm:

$$
\|f\|:=\sup _{b \in \Phi}|f(b)| \text {. }
$$

This implies $f_{j}(T)\left(i_{j} I\right)=\left(i_{j} I\right) f_{j}(T)$ for each $j$ due to the $\mathcal{A}_{v}$-gradation of the projection valued measure $\hat{\boldsymbol{E}}$ and due to the commutativity of the complex field $\boldsymbol{R} \oplus \boldsymbol{R} i_{j}$ for each $j \geq 1$. Therefore, decompositions $(4-6)$ extend the $\sigma$-normal-homomorphism from $\theta: \boldsymbol{B}(\Phi, \boldsymbol{R})$ э $f \mapsto f(T) \in \boldsymbol{C}_{0}$ up to $\theta: \boldsymbol{B}\left(\Phi, \mathcal{A}_{v}\right)$ э $f \mapsto f(T) \in \boldsymbol{C}$.

For a self-adjoint operator $T$ its spectrum $s p(T)$ is contained in $\alpha \boldsymbol{R}$, while the real field $\boldsymbol{R}$ is separable as the normed space.

16) Theorem. Let $\boldsymbol{A}$ and $\boldsymbol{B}$ be $C^{*}$-algebras over the Cayley-Dickson algebra $\mathcal{A}_{v}, 2 \leq v$, let also $\theta: \boldsymbol{A} \rightarrow \boldsymbol{B}$ be a-homomorphism. Then

1) $s p(\theta(T)) \subset \theta(\operatorname{sp}(T))$ and $\|\theta(T)\| \leq\|T\|$ for each $T \in \boldsymbol{A}$;

2) if $K$ is a self-adjoint element in $\boldsymbol{A}$ and $f \in C\left(\operatorname{sp}(K), \mathcal{A}_{v}\right)$ and $g \in C\left(\theta(\operatorname{sp}(K)), \mathcal{A}_{v}\right)$ and $\theta \circ f(b)=g(b)$ for each $b \in \boldsymbol{R}$, then $\theta(f(K))=g(\theta(K))$;

3) if $\theta$ is a-isomorphism, then $\|\theta(T)\|=\|T\|$ and $\operatorname{sp}(\theta(T))=\theta(\operatorname{sp}(T))$ for each $T \in \boldsymbol{A}$ and $\theta(\boldsymbol{A})$ is a $C^{*}$-subalgebra in $\boldsymbol{B}$.

Proof. 1). Suppose that $T \in \boldsymbol{A}$ and $a \notin s p(T)$, then $(a I-T)$ has an inverse $Q$ in $\boldsymbol{A}$. This implies

$\theta(a) \notin s p(\theta(T))$, since $\theta(I)=I$ and $\theta(a I-T)=\theta(a) I-\theta(T)$ has an inverse $\theta(Q)$ in $\boldsymbol{B}$, hence $\operatorname{sp}(\theta(T)) \subset \theta(\operatorname{sp}(T))$, where $I$ denotes the unit element in $\boldsymbol{A}$ and in $\boldsymbol{B}$. Then

$$
\|T\|^{2}=\left\|T^{*} T\right\|=\left|\sigma\left(T^{*} T\right)\right|
$$

and

$$
\|\theta(T)\|^{2}=\left\|\theta\left(T^{*}\right) \theta(T)\right\|=\left|\sigma\left(\theta\left(T^{*} T\right)\right)\right|
$$

(see $\$ 2.24$ [18]). But $\left|\sigma\left(\theta\left(T^{*} T\right)\right)\right| \leq\left|\sigma\left(T^{*} T\right)\right|$, since sp $(\theta(T)) \subset \theta(\operatorname{sp}(T))$, consequently, $\|\theta(T)\| \leq\|T\|$ (see also Lemma 2.26 [18]). That is the-homomorphism $\theta$ is continuous.

2) If an element $K$ is self-adjoint, then $s p(K) \subset \boldsymbol{R}$. At the same time one has $\theta(b)=b$ for each $b \in \boldsymbol{R}$, since $\theta(b I)=b I$ for real $b$. If $\left\{P_{n}: n\right\}$ is a sequence of polynomials tending to $f$ uniformly on $\operatorname{sp}(K)$, then

$$
\lim _{n} \theta\left(P_{n}(K)\right)=\theta(f(K))
$$

and

$$
\lim _{n} P_{n}(\theta(K))=g(\theta(K)),
$$

since the restriction $\left.\theta\right|_{\mathcal{A}_{v} I}$ induces a-homomorphism from $\mathcal{A}_{v}$ into $\mathcal{A}_{v}$.

3) If $\theta$ is a-isomorphism and $K$ is a self-adjoint element in $\boldsymbol{A}$, then by (1) we have $\operatorname{sp}(\theta(K)) \subset \theta(\operatorname{sp}(K))$. If this inclusion would be strict, then there would exist a non-zero element $f \in C\left(\operatorname{sp}(K), \mathcal{A}_{v}\right)$ such that $\left.f\right|_{s p(\theta(K))}=0$. But (2) means that $f(K) \neq 0$ and $\theta(f(K))=g(\theta(K))=0$, contrary to the assumption that $\theta$ is one-to-one. Thus $s p(\theta(K))=\theta(\operatorname{sp}(K))$ and $|\sigma(\theta(K))|=|\sigma(K)|$ for each self-adjoint element $K$. Particularly, this is accomplished for $K=T^{*} T$ and from (1) it follows that

$$
\|T\|^{2}=\left|\sigma\left(T^{*} T\right)\right|=\left|\sigma\left(\theta\left(T^{*} T\right)\right)\right|=\|\theta(T)\|^{2},
$$

consequently, $\|T\|=\|\theta(T)\|$, where $T \in \boldsymbol{A}$ is an arbitrary element. On the other hand, $\boldsymbol{A}$ is a complete normed space and $\theta$ is the-isometry, consequently, $\theta(\boldsymbol{A})$ is closed in $\boldsymbol{B}$ and contains $I$. That is $\theta(\boldsymbol{A})$ is a $C^{*}$-subalgebra in B.

If $V$ is an element in $\boldsymbol{B}$, it induces a quasi-commutative $C^{*}$-sub-algebra $c l_{B}\left[\operatorname{alg}_{\mathcal{A}_{v}}\left(V, V^{*}, I\right)\right]=: Y$ over $\mathcal{A}_{v}$ generated by $V, V^{*}$ and $T$. This sub-algebra has the decomposition $\boldsymbol{Y}=\boldsymbol{Y}_{0} i_{0} \oplus \cdots \oplus \boldsymbol{Y}_{m} i_{m} \oplus \cdots$, where $\boldsymbol{Y}_{0}, \cdots, \boldsymbol{Y}_{m}, \cdots$ are isomorphic algebras over $\boldsymbol{R}$. Now take the restricted subalgebra $\boldsymbol{Y}_{\mathcal{A}_{r}}=\boldsymbol{Y}_{0} i_{0} \oplus \cdots \oplus \boldsymbol{Y}_{2^{r}-1} i_{2^{r}-1}$, where

$2 \leq r \leq \min (3, v)$. More generally take sub-algebras ${ }^{t} \boldsymbol{Y}$, where $\Psi_{t}$ is isomorphic with $\mathcal{A}_{r}$ and impose Conditions $2.9(M 1-M 3)$. To the latter $C^{*}$-algebras ${ }^{t} \boldsymbol{Y}$ Theorem 2.24 [18] is applicable. In view of this theorem $s p_{B}(\theta(T))=s p_{\theta(A)}(\theta(T))$, consequently,

$\theta(s p(T))=s p_{\theta(A)}(\theta(T))$, since $\theta$ is the-isomorphism, where $s p_{B}(P)$ denotes the spectrum of an element $P$ in B.

17) Lemma. Let $\theta: \boldsymbol{A} \rightarrow \boldsymbol{B}$ be a-homomorphism of $C^{*}$-algebras over the Cayley-Dickson algebra $\mathcal{A}_{v}, 2 \leq v$. Then the testriction $\left.\theta\right|_{\mathcal{A}_{v} I}$ induces a-iosmorphism of $\mathcal{A}_{v}$ into $\mathcal{A}_{v}$

Proof. A-homomorphism $\theta$ is $\boldsymbol{R}$-linear and $\mathcal{A}_{v}$-additive and $\theta\left(T^{*}\right)=[\theta(T)]^{*}$ so the restricrion $\left.\theta\right|_{\mathcal{A}_{v} I}$ also has there properties. This provides a-homomorphism from $\mathcal{A}_{v}$ into $\mathcal{A}_{v}$. Since

$$
\theta(z)=\theta(z 1)=\theta(1 z)=\theta(z) \theta(1)=\theta(1) \theta(z),
$$

the image of 1 is $1, \theta(1)=1$. Due to the $\boldsymbol{R}$-linearity this means that $\theta(b)=\theta(b 1)=b 1=b$ for each real number $b \in \boldsymbol{R}$, i.e. $\left.\theta\right|_{\boldsymbol{R}}=i d$. For each purely imaginary Cayley-Dickson number $M \in \mathcal{A}_{v}, \operatorname{Re}(M)=0$, the identities $-M^{2}=M M^{*}=|M|^{2}$ are satisfied, consequently, 
$\theta\left(-M^{2}\right)=\theta\left(|M|^{2}\right)=|M|^{2}=-M^{2}$ and $\theta\left(M^{2}\right)=M^{2}$.

If $M$ and $K$ are two Cayley-Dickson numbers which are orthogonal $\operatorname{Re}\left(M K^{*}\right)=0$, then

$$
\begin{aligned}
\theta(0) & =\theta\left(2 \operatorname{Re}\left(M K^{*}\right)\right) \\
& =\theta\left(M K^{*}+K M^{*}\right)=\theta\left(M K^{*}\right)+\theta\left(K M^{*}\right) \\
& =\theta(M)[\theta(K)]^{*}+\theta(M)[\theta(M)]^{*} \\
& =2 \operatorname{Re}\left(\theta(M)[\theta(K)]^{*}\right)=0 .
\end{aligned}
$$

Therefore, $\theta\left(i_{j}\right)$ and $\theta\left(i_{k}\right)$ are orthogonal for each $k \neq j \geq 0$ and $\left|\theta\left(i_{j}\right)\right|=1$ and $\theta\left(b i_{j}\right)=b \theta\left(i_{j}\right)$ for every real number $b \in \boldsymbol{R}$, also $\left(\theta\left(i_{j}\right)\right)^{2}=1$ for each $j \geq 1$. Moreover, we infer that

$$
\theta\left(i_{j} i_{k}\right)=\theta\left(-i_{k} i_{j}\right)=\theta\left(i_{j}\right) \theta\left(i_{k}\right)=-\theta\left(i_{k}\right) \theta\left(i_{j}\right)
$$

for each $j \neq k \geq 1$. That is, $\operatorname{span}_{\boldsymbol{R}}\left\{\theta\left(i_{j}\right): j=0, \cdots, 2^{r}-1\right\}$ is-isomorphic with $\mathcal{A}_{v}$ fore each $2 \leq r<\infty, r \leq v$. Using embedded sub-algebras $\Psi_{t}$ satisfying Condition 2.9(M1) into $\mathcal{A}_{v}$ we get that $\theta\left(\mathcal{A}_{v}\right)$ is-isomorphic with $\mathcal{A}_{v}$, since

$$
\theta\left(z z^{*}\right)=\theta(z)[\theta(z)]^{*}=\theta\left(|z|^{2}\right)=|z|^{2}=|\theta(z)|^{2}
$$

and hence $|\theta(z)|=|z|$ for each Cayley-Dickson number $z \in \mathcal{A}_{v}$.

18) Remark. Lemma 17 means that up to a-isomorphic of the Cayley-Dickson algebra $\mathcal{A}_{v}$ one can considerhomomorphisms $\theta$ satisfying additional restriction: $\left.\theta\right|_{\mathcal{A}_{v} I}=i d$, that is $\theta(a)=a$ for each $a \in \mathcal{A}_{v}$. This will simplify notations, for example, $\theta(s p(T))=s p(T)$, also $f=g$ in Theorem 16(2), i.e.

$\theta(f(K))=f(\theta(K))$.

19) Theorem. If $T$ is a bounded normal $\mathcal{A}_{r}$ meta-invariant operator on an $\mathcal{A}_{v}$ Hilbert space $X, 2 \leq v \leq \aleph_{0}$, $1 \leq r \leq \min (3, v)$. Suppose that $\theta: \boldsymbol{B}\left(\operatorname{sp}(T), \mathcal{A}_{v}\right) \rightarrow \boldsymbol{A}$ is a $\sigma$-normal homomorphism into a quasi-commutative von Neumann algebra over $\mathcal{A}_{v}$ such that $\theta(1)=I$ and $\theta(i d)=T$, where id $(b)=b$ for each $b \in \operatorname{sp}(T)$. Then $\theta\left(\boldsymbol{B}\left(\operatorname{sp}(T), \mathcal{A}_{v}\right)\right) \subset \boldsymbol{K}$, where

$\boldsymbol{K}=\operatorname{cl}\left[\operatorname{alg}_{\mathcal{A}_{v}}\left(T, T^{*}, I\right)\right]$ is the quasi-commutative von Neumann algebra over $\mathcal{A}_{v}$ generated by $T$ and $T^{*}$ and $I$ so that $\theta(f)=f(T)$ for every $f \in \boldsymbol{B}\left(\operatorname{sp}(T), \mathcal{A}_{v}\right)$.

Proof. As in $\S 15 \boldsymbol{B}\left(s p(T), \mathcal{A}_{v}\right)$ is supplied with the structure of a quasi-commutative $C^{*}$-algebra with $s p(T)$ instead of $\Phi$. On the other hand, the inclusion

$s p(T) \subset \mathcal{A}_{v}$ is valid and 1 , id $\in \boldsymbol{B}\left(\operatorname{sp}(T), \mathcal{A}_{v}\right)$. For each $f \in \boldsymbol{B}\left(s p(T), \mathcal{A}_{v}\right)$ and $a \in \mathcal{A}_{v} \backslash s p(f)$ there exists $h \in \boldsymbol{B}\left(\operatorname{sp}(T), \mathcal{A}_{v}\right)$ such that $(f-a 1) h=1$, consequently, $(\theta(f)-\theta(a) I) \theta(h)=I$ and hence $\theta(a) \notin s p(\theta(f))$. If $g \in \boldsymbol{B}(\operatorname{sp}(T), \boldsymbol{R})$, then $(g-a 1)$ has an inverse $h$ in $\boldsymbol{B}\left(s p(T), \mathcal{A}_{v}\right)$ for each $a \in \mathcal{A}_{v} \backslash \boldsymbol{R}$, consequently, $\theta(g)$ is self-adjoint in $\boldsymbol{A}$. The homomorphism $\theta$ is order preserving and does not increase norm by Theorem 18 .

Next we apply Theorems 2.24 and 3.22 [17] and \$2.9. Consider $\theta$ restricted to $C\left(\operatorname{sp}(T), \mathcal{A}_{v}\right)$. Then $\theta(f)=f(T)$ for each $f \in C\left(\operatorname{sp}(T), \mathcal{A}_{v}\right)$ and $f(T) \in \mathrm{cl}\left[\operatorname{alg}_{\mathcal{A}_{v}}\left(T, T^{*}, I\right)\right]$. The mappings $\theta$ and $g \mapsto g(T)$ are $\sigma$-normal, as in $\S \S 14,15$ we get that $\theta(g)=g(T) \in \boldsymbol{K}$, when $g=\chi_{U}$ is the characteristic function of an open subset $U$ in $\operatorname{sp}(T)$.

We consider the family $\mathcal{C}$ of all Borel subsets with their characteristic functions satisfying the equality $\theta(g)=g(T)$. By the $\sigma$-normality this family $\mathcal{C}$ contains the union of each countable subfamily. It contains also the complement of each set, since $\theta(1)=I$. Thus $\mathcal{C}$ contains the family $\mathcal{B}(s p(T))$ of all Borel subsets of $\operatorname{sp}(T)$ and $\theta\left(\chi_{U}\right)=\chi_{U}(T)$ for each $U \in \mathcal{B}(\operatorname{sp}(T))$. The mapping $\theta$ is $\boldsymbol{R}$-linear and $\mathcal{A}_{v}$ additive and norm continuous so that $\|g(T)\| \leq\|g\|$. Due to the meta-invariance of the operator $T$ the set of all step functions is dense in $\boldsymbol{B}\left(\operatorname{sp}(T), \mathcal{A}_{v}\right)$ relative to the norm topology on it, since $s p\left(\left.T\right|_{t_{X}}\right)$ is compact for each $t \in \Upsilon$, while $c l\left[\bigcup_{t \in \Upsilon} s p\left(\left.T\right|_{t_{X}}\right)\right]=s p(T) \subset \mathcal{A}_{v}$ and the Cayley-Dickson algebra $\mathcal{A}_{v}$ is separable as the normed space for $v \leq \aleph_{0}$. Therefore $\theta(g)=g(T) \in \boldsymbol{K}$ for each $g \in \boldsymbol{B}\left(\operatorname{sp}(T), \mathcal{A}_{v}\right)$.

20) Theorem. Let $T$ be a symmetrical $\mathcal{A}_{k}$ metainvariant operator, $2 \leq k \leq \min (v, 3)$ and $a \in \mathcal{A}_{v} \backslash \boldsymbol{R} 1$, then there exists its resolvent function $\boldsymbol{R}(a ; T)$ and $|x| \leq 2|R(a ; T) x| /|a-\tilde{a}|$ for each $x \in \mathcal{D}(T)$. Let $T$ be a closed operator, then the sets $\rho(T), \sigma_{p}(T)$, $\sigma_{c}(T)$ and $\sigma_{r}(T)$ are not intersecting and their union is the entire Cayley-Dickson algebra $\mathcal{A}_{v}$,

$$
\sigma(T)=\sigma_{p}(T) \cup \sigma_{c}(T) \cup \sigma_{r}(T) \subset \mathcal{A}_{v} .
$$

Moreover, if additionally an operator $T$ is self-adjoint and quasi-linear, then the inclusion $\sigma(T) \subset \boldsymbol{R} 1$ is satisfied and $R(a ; T)^{*}=R\left(a^{*} ; T\right)$.

Proof. In the general case for a quasi-linear operator (not necessarily symmetrical) for the decomposition of the components $\mu_{i_{s}, i_{l}}(\mathrm{~d} \lambda ; x, y)$ of the projection valued measure $\hat{\mu}(x, y) \cdot f$ defined (see also $\$ 2.23$ [18]) as the sum of the point $\left(\mu_{i_{s}, i_{l}}\right)_{p}$, absolutely continuous $\left(\mu_{i_{s}, i_{l}}\right)_{a c}$ and continuous singular $\left(\mu_{i_{s}, i_{l}}\right)_{\text {sing }}$ measures in accordance with the Lebesgue theorem cited in $\$ 2.50$ gives $L^{2}\left(\mathcal{A}_{k}, \mu_{i_{s}, i_{l}}, \mathcal{A}_{k}\right)=\mathcal{H}_{p, s, l} \oplus \mathcal{H}_{a c, s, l} \oplus \mathcal{H}_{\text {sing, }, l}$, where

$$
\begin{aligned}
\mathcal{H}_{p, s, l} & :=L^{2}\left(\mathcal{A}_{k},\left(\mu_{i_{s}, i_{l}}\right)_{p}, \mathcal{A}_{k}\right), \\
\mathcal{H}_{a c, s, l} & :=L^{2}\left(\mathcal{A}_{k},\left(\mu_{i_{s}, i_{l}}\right)_{a c}, \mathcal{A}_{k}\right)
\end{aligned}
$$


and

$$
\mathcal{H}_{\text {sing,s, },}:=L^{2}\left(A_{k},\left(\mu_{i_{s}, i_{l}}\right)_{\text {sing }}, \mathcal{A}_{k}\right) .
$$

At the same time for a symmetrical operator, the inclusion $\sigma(T) \subset \boldsymbol{R}$ is satisfied and due to the relations given in $\$ 2.23$ [18] for components of the projection valued measure the supports of all these measures for different $s, l$ are consistent and are contained in $\boldsymbol{R}$.

21) Theorem. For a self-adjoint quasi-linear $\mathcal{A}_{k}$ meta-invariant operator $T$ in a Hilbert space $X$ over the Cayley-Dickson algebra $\mathcal{A}_{v}, 2 \leq v$,

$1 \leq k \leq \min (3, v)$, there exists a uniquely defined regular countably additive self-adjoint spectral measure $\hat{\boldsymbol{E}}$ on $\mathcal{B}\left(\mathcal{A}_{v}\right),\left.\hat{\boldsymbol{E}}\right|_{\rho(T)}=0$ such that

1) $\mathcal{D}(T):=\left\{x: x \in X ; \int_{\sigma(T)}\left\langle\left(\hat{\boldsymbol{E}}(\mathrm{d} z) \cdot z^{2}\right) x ; x\right\rangle<\infty\right\}$ and

2) $T x=\lim _{n \rightarrow \infty} \int_{-n}^{n}(\hat{\boldsymbol{E}}(\mathrm{d} z) \cdot z) x$

for each $x \in \mathcal{D}(T)$.

Proof. Due to Proposition 2.22.9, $\$ 2.29$ and Theorem 2.30 [18] the space $\mathcal{D}(T)$ is $\mathcal{A}_{v}$-vector. Use Lemma 2.26 [18] and take a marked element $q \in\left\{i_{1}, \cdots, i_{2^{k}-1}\right\}$, then we consider $h(z):=(q-z)^{-1}$ from $\mathcal{A}_{k}$ into ${ }^{\mathcal{A}_{k}}$. It also is the homomorphism of the unit sphere $S^{2^{k}-1}:=\left\{z \in \mathcal{A}_{k}:|z|=1\right\}$ into $\mathcal{A}_{k}$ and for

$$
A:=((q-z) I)(R(z ; T)((q-z) I))+(q-z) I
$$

for each $z \in \rho(T) \backslash\{q\} \quad$ is accomplished the identity $(h I-R(q ; T)) A=I$ in accordance with Lemma 2.5 [18]. If $z=q$, then $p:=h(q)=\infty$, consequently, $p \notin \sigma(R(q ; T))$.

Let $0 \neq p=h(z) \in \rho(R(q ; T))$, then there exists $B:=R(q ; T) A$, where $A:=(p I-R(q ; T))^{-1}$, consequently, $B$ is bijective, $\mathcal{R}(B)=\mathcal{D}(T)$ and $(z I-T) B=(q-z) I$, that is, $z \in \rho(T)$. For $\mu=h(z)=0 \in \rho(R(q ; T))$ the operator

$R(q ; T)^{-1}=(q I-T)$ is bounded and it is defined everywhere and this case was considered in Theorem 2.24 [18]. Therefore, the mapping $h$ is the homeomorphism from $\rho(T)$ onto $\rho(R(q ; T)) \cup\{\infty\}$, consequently, also from $\sigma(T) \cap\{\infty\}$ onto $\sigma(R(q ; T))$.

If $M \in \mathcal{S}_{k}$ and $i_{l} \in \mathcal{S}_{k}$, then $\operatorname{alg}_{R}\left(M, i_{l}\right)$ is associative and $\operatorname{alg}_{R}\left(M, i_{l}, q\right)$ is alternative, since $k \leq 3$, while a purely imaginary quaternion or octonion number has the decomposition $M=\alpha i_{l}+\beta K$, where $\alpha, \beta \in \boldsymbol{R}$, $\operatorname{Re}\left(i_{l} K^{*}\right)=0, K \in \mathcal{S}_{k}$. If $M, q, i_{l} \in \mathcal{S}_{k}$, then $\operatorname{alg}_{R}\left(M, i_{l}, q\right)$ is alternative as the subalgebra contained in $\mathcal{A}_{k}, \quad k \leq 3$. If $M \in \mathcal{S}_{k}$ and $q, i_{l} \in \mathcal{S}_{v} \backslash \mathcal{S}_{k}$, then $M$ anti-commutes with $q$ and $i_{l}$, also $M$ is orthogonal to $q$ and $i_{l}$, consequently, $\operatorname{alg}_{R}\left(M, i_{l}, q\right)$ is alternative in this case also. Therefore, the subalgebra $\operatorname{alg}_{R}\left(b, q, i_{l}\right)$ is alternative for each $b \in \mathcal{A}_{k}$ and $q \in\left\{i_{1}, i_{2}, \cdots\right\}$.

For each $\delta \in \mathcal{B}\left(\mathcal{A}_{k}\right)$ put $\hat{\boldsymbol{E}}(\delta):=\hat{\boldsymbol{E}}^{1}(h(\delta))$, where $\hat{\boldsymbol{E}}^{1}$ is the $\mathcal{A}_{v}$-graded decomposition of the unity for a normal operator $R(q ; T)$. On the other hand, each vector $x \in X$ has the decomposition $x=x_{0} i_{0}+x_{1} i_{1}+\cdots$ and projection valued measure $\hat{\boldsymbol{E}}$ is $\mathcal{A}_{v}$ graded, where $x_{l} \in X_{l}$ for each $l$ (see $\$ 2.8$ ).

Mention that $\hat{\boldsymbol{E}}^{1}(\{0\})=0$, since in the contrary case

$$
R(q ; T) x=\int_{\{0\}} b \hat{\boldsymbol{E}}^{1}(\mathrm{~d} b) x=0
$$

for each $x \in X$ such that $0 \neq x=\hat{\boldsymbol{E}}^{1}(\{0\}) x$ contradicting the invertibility of $R(q ; T)$. Therefore,

$\hat{\boldsymbol{E}}\left(\mathcal{A}_{k}\right)=I$, consequently, $\hat{\boldsymbol{E}}$ is an $\mathcal{A}_{v}$ graded spectral measure. This spectral measure is self-adjoint, countably additive and regular due to such properties of $\hat{\boldsymbol{E}}^{1}$ (see Theorem 15). Then $\hat{\boldsymbol{E}}(\sigma(T))=I$ and $\hat{\boldsymbol{E}}(\delta)=0$ for $\delta \subset \rho(T)$, where $\sigma(T) \subset \boldsymbol{R}$. We now put

$$
\mathcal{G}=\left\{x \in X: \int_{\sigma(T)} b^{2}\langle\hat{\boldsymbol{E}}(\mathrm{d} b) x ; x\rangle<\infty\right\} .
$$

Evidently, $\hat{\boldsymbol{E}}(\delta) X \subset \mathcal{G}$ for each bounded Borel subset $\delta$ contained in $\boldsymbol{R}$, since

$$
\int_{\sigma(T)} b^{2}\langle\hat{\boldsymbol{E}}(\mathrm{d} b) x ; x\rangle=\int_{-\infty}^{\infty} b^{2}\langle\hat{\boldsymbol{E}}(\mathrm{d} b) x ; x\rangle
$$

and

$$
\left|\int_{\delta} b \hat{\boldsymbol{E}}(\mathrm{d} b) x\right|^{2}=\int_{\delta} b^{2}\langle\hat{\boldsymbol{E}}(\mathrm{d} b) x ; x\rangle .
$$

Thus the equality

$$
\text { 1) } \mathcal{G}=\left\{x: \exists \lim _{n \rightarrow \infty} \int_{-n}^{n} b \hat{\boldsymbol{E}}(\mathrm{d} b) x\right\}
$$

is fulfilled.

We next verify, that $\mathcal{D}(T) \subset \mathcal{G}$. Take an arbitrary vector $x \in \mathcal{D}(T)$. From the definition we have the formula $\mathcal{D}(T)=R(q ; T) X$, consequently, a vector $y \in X$ exists such that $x=R(q ; T) y$ and

$$
\int_{-n}^{n} b \hat{\boldsymbol{E}}(\mathrm{d} b) x=\int_{-n}^{n} b \hat{\boldsymbol{E}}(\mathrm{d} b) R(q ; T) y .
$$

Making the change of measure we infer the relations:

$$
\text { 2) } R(q ; T) y=\int_{A_{k}} \hat{\boldsymbol{E}}^{1}(\mathrm{~d} p) \cdot p y=\int_{A_{k}} \hat{\boldsymbol{E}}(\mathrm{d} b) \cdot \frac{1}{q-b} y \text {, }
$$

consequently,

$$
\int_{-n}^{n} b \hat{\boldsymbol{E}}(\mathrm{d} b) x=\int_{-n}^{n} \hat{\boldsymbol{E}}(\mathrm{d} b) \cdot \frac{b}{q-b} y .
$$

This demonstrates that $x \in \mathcal{G}$ and hence $\mathcal{D}(T) \subset \mathcal{G}$. In view of Formula (2) and Theorem 15 we have 


$$
R(q ; T) \int_{-n}^{n} \hat{\boldsymbol{E}}(\mathrm{d} b) \cdot(q-b)=\hat{\boldsymbol{E}}([-n, n]),
$$

consequently, $\hat{\boldsymbol{E}}([-n, n]) X \subset \mathcal{D}(T)$. At the same time one has:

$$
\begin{aligned}
T \hat{\boldsymbol{E}}([-n, n]) & =[q I-(q I-T)]\left\{R(q ; T) \int_{-n}^{n} \hat{\boldsymbol{E}}(\mathrm{d} b)(q-b)\right\} \\
& =\int_{-n}^{n} b \hat{\boldsymbol{E}}(\mathrm{d} b)
\end{aligned}
$$

and this together with (1) implies that the sequence $\{T \hat{\boldsymbol{E}}([-n, n]) x: n\}$ converges for each $x \in \mathcal{G}$. The operator $T$ is closed and $\lim _{n \rightarrow \infty} \hat{\boldsymbol{E}}([-n, n]) x=x$, consequently, $x \in \mathcal{D}(T)$ and $T x=\lim _{n} \int_{-n}^{n} b \hat{\boldsymbol{E}}(\mathrm{d} b) x$. Thus $\mathcal{G} \subset \mathcal{D}(T)$ and together with the opposite inclusion demonstrated above this gives the equality $\mathcal{G}=\mathcal{D}(T)$.

Suppose that another $\mathcal{A}_{v}$ graded measure $\hat{\boldsymbol{F}}$ exists with the same properties as $\hat{\boldsymbol{E}}$. In accordance with statement $(a)$ of this theorem we have

$\hat{\boldsymbol{F}}([-m, m]) x \in \mathcal{D}(T)$ and together with $(b)$ this implies that

$$
\begin{aligned}
& (q I-T) \hat{\boldsymbol{F}}([-m, m]) x \\
& =\lim _{n \rightarrow \infty} \int_{-n}^{n} F(\mathrm{~d} b) \cdot(q-b)\{\hat{\boldsymbol{F}}([-m, m]) x\} \\
& =\int_{-m}^{m} \hat{\boldsymbol{F}}(\mathrm{d} b) \cdot(q-b) x .
\end{aligned}
$$

In accordance with Theorems 15 above and 2.24, Propositions 2.22.20, 2.22.12, 2.22.18 [18] one has

$$
\hat{\boldsymbol{F}}([-m, m])=\left\{\int_{-m}^{m} \hat{\boldsymbol{F}}(\mathrm{d} b) \cdot(q-b)\right\}\left\{\int_{-m}^{m} \hat{\boldsymbol{F}}(\mathrm{d} b) \cdot \frac{1}{q-b}\right\}
$$

and

$$
\begin{aligned}
\hat{\boldsymbol{F}}([-m, m]) & =(q I-T)\left\{\hat{\boldsymbol{F}}([-m, m]) \int_{-m}^{m} \hat{\boldsymbol{F}}(\mathrm{d} b) \cdot \frac{1}{q-b}\right\} \\
& =(q I-T) \int_{-m}^{m} \hat{\boldsymbol{F}}(\mathrm{d} b) \cdot \frac{1}{q-b},
\end{aligned}
$$

consequently,

$$
R(q ; T) \hat{\boldsymbol{F}}([-m, m])=\int_{-m}^{m} \hat{\boldsymbol{F}}(\mathrm{d} b) \cdot \frac{1}{q-b} .
$$

Taking the limit with $m$ tending to the infinity and substituting the measure one gets:

$$
R(q ; T)=\int_{-\infty}^{\infty} \hat{\boldsymbol{F}}(\mathrm{d} b) \cdot \frac{1}{q-b}=\int_{A_{k}} \hat{\boldsymbol{F}}\left(h^{-1}(\mathrm{~d} b)\right) \cdot b,
$$

consequently, $\hat{\boldsymbol{F}}\left(h^{-1}(\delta)\right)=\hat{\boldsymbol{E}}^{1}(\delta)$ and hence
$\hat{\boldsymbol{F}}(\delta)=\hat{\boldsymbol{E}}(\delta)$ for each Borel subset $\delta$ contained in $\mathcal{A}_{k}$. Thus the $\mathcal{A}_{v}$ graded spectral measure is unique.

22) Definitions. A unique (graded) spectral measure, related with a self-adjoint quasi-linear operator $T$ in $X$ is called a decomposition of the unity for $T$. For an $\mathcal{A}_{v}$ valued Borel function $f$ defined $\hat{\boldsymbol{E}}$-almost everywhere on $\boldsymbol{R}$ a function $f(T)$ of a self-adjoint operator $T$ is defined by the relations:

1) $\mathcal{D}(f(T)):=\left\{x\right.$ : there exists $\left.\lim _{n} f_{n}(T) x\right\}$, where $f_{n}(z):=f(z)$ for $|f(z)| \leq n ; f_{n}(z):=0$ while $|f(z)|>n$;

2) $f(T) x:=\lim _{n} f_{n}(T) x$ for each $x \in \mathcal{D}(f(T))$, where $n \in N$.

23) Theorem. Let $\hat{\boldsymbol{E}}$ be a decomposition of the unity for a self-adjoint quasi-linear $\mathcal{A}_{r}$-meta-invariant operator $T$ in a Hilbert space $X$ over the Cayley-Dickson algebra $\mathcal{A}$, and let a Borel function $f$ be as in §22, $2 \leq v, 1 \leq r \leq \min (3, v)$. Then $f(T)$ is a closed quasilinear operator with an everywhere dense domain of its definition, moreover:
a) $\mathcal{D}(f(T))=\left\{x: \int_{-\infty}^{\infty}|f(z)|^{2}\langle\hat{\boldsymbol{E}}(\mathrm{d} z) x ; x\rangle<\infty\right\}$;
b) $\langle f(T) x ; y\rangle=\int_{-\infty}^{\infty}\langle\hat{\boldsymbol{E}}(\mathrm{d} z) \cdot f(z) x ; y\rangle, x \in \mathcal{D}(f(T))$;
c) $|f(T) x|^{2}=\int_{-\infty}^{\infty}|f(z)|^{2}\langle\hat{\boldsymbol{E}}(\mathrm{d} z) x ; x\rangle, x \in \mathcal{D}(f(T))$;
d) $f(T)^{*}=\tilde{f}(T)$;
e) $R(q ; T)=\int_{-\infty}^{\infty} \hat{\boldsymbol{E}}(\mathrm{d} z) \cdot \frac{1}{(q-z)}, q \in \rho(T)$.

Proof. Take a sequence of functions $f_{n}$ from $\$ 22$ and subsets $\delta_{n}:=\{z:|f(z)| \leq n\}$. Then

$$
\begin{aligned}
|f(T) x|^{2} & =\lim _{n}\left|f_{n}(T) x\right|^{2}=\lim _{n} \int_{\delta_{n}}|f(z)|^{2}\langle\hat{\boldsymbol{E}}(\mathrm{d} z) x ; x\rangle \\
& =\int_{-\infty}^{\infty}|f(z)|^{2}\langle\hat{\boldsymbol{E}}(\mathrm{d} z) x ; x\rangle
\end{aligned}
$$

for each $x \in \mathcal{D}(f(T))$, from this it follows $(c)$ and that $\mathcal{D}(f(T))$ is contained in

$$
\left\{x: \int_{-\infty}^{\infty}|f(z)|^{2}\langle\hat{\boldsymbol{E}}(\mathrm{d} z) x ; x\rangle<\infty\right\} .
$$

$$
\begin{aligned}
& \text { If } y \in\left\{x: \int_{-\infty}^{\infty}|f(z)|^{2}\langle\hat{\boldsymbol{E}}(\mathrm{d} z) x ; x\rangle<\infty\right\} \text {, then } \\
& \left|f_{m}(T) y-f_{n}(T) y\right|^{2}=\int_{\delta_{m} \backslash \delta_{n}}|f(z)|^{2}\langle\hat{\boldsymbol{E}}(\mathrm{d} z) x ; x\rangle<\infty
\end{aligned}
$$

for each $m>n$, consequently,

$$
\limsup _{n \rightarrow \infty}\left|f_{m>n}(T) y-f_{n}(T) y\right|=0 .
$$

This demonstrates statement a). 
To the non-commutative measure $\hat{\mu}$ given on a $\sigma$ algebra $\Phi$ of subsets of the set $\mathcal{S}$ a quasi-linear operator with values in $\mathcal{A}$, corresponds and due to Theorem 2.24 and Definitions 2.23 [18] this measure is completely characterized by the family of $\boldsymbol{R}$-valued measures $\mu_{i_{j}, i_{l}}$, such that

$$
\hat{\mu}(x, y) \cdot f=\sum_{k, l} \mu_{i_{k}, i_{l}}\left({ }_{k} f ; x, y\right) i_{l},
$$

where $f=\sum_{k} f i_{k}$ with real-valued functions ${ }_{k} f$,

$$
\mu_{i_{k}, i_{l}}{ }^{k}\left({ }_{k} f ; x, y\right)=\int_{\mathcal{S}} f(\lambda) \mu_{i_{k}, i_{l}}(\mathrm{~d} \lambda ; x, y)
$$

for $x, y \in \mathcal{D}(f(T))$, for each $\hat{\mu}$-integrable $\mathcal{A}_{v}$-valued function $f$ with components ${ }_{k} f$, where

$k, l=0,1, \cdots, m, \cdots$. Then it can be defined the variation of the measure

$$
V(\hat{\mu}, U):=\sup _{W_{l} \subset U} \sum_{l}\left|\hat{\mu}\left(\chi_{W_{l}}\right)\right|
$$

by all finite disjoint systems $\left\{W_{l}\right\}$ of subsets $W_{l} \in \Phi$ in $U$ with $\bigcup W_{l}=U$.

We have the embedding $\mathcal{A}_{r} \longmapsto \mathcal{A}_{v}$. Here we consider the Borel $\sigma$-algebra $\Phi=\mathcal{B}\left(\mathcal{A}_{v}\right)$ on the CayleyDickson algebra $\mathcal{A}_{v}$. Mention that each Borel measure $\hat{\mu}$ on $\mathcal{A}_{r}$ has a natural extension to a Borel measure on $\mathcal{A}_{v}$ so that $V\left(\hat{\mu}, \mathcal{A}_{v} \backslash \mathcal{A}_{v}\right)=0$. If $\hat{\mu}$ is bounded, then it is the quasi-linear operator of the bounded variation with

$$
V(\hat{\mu}, \mathcal{S}) \leq \sup _{U \in \Phi}\left|\hat{\mu}\left(\chi_{U}\right)\right|,
$$

moreover, $V(\hat{\mu}, *)$ is $\sigma$-additive on $\Phi$, if $\hat{\mu}$ is $\sigma$-additive, that is in the case considered here with $\mathcal{S}=\mathcal{A}_{v}$ and $\Phi=\mathcal{B}\left(\mathcal{A}_{v}\right)$.

The function $f$ we call $\hat{\mu}$-measurable, if each its component $f_{j}$ is $\mu_{i_{j}, i_{l}}$-measurable for each $j$ and $l=0,1, \cdots, m, \cdots$ The space of all $\hat{\mu}$-measurable $\mathcal{A}_{v}$ valued functions $f$ with

$$
V\left(\hat{\mu},|f|^{p}\right)^{1 / p}=:|f|_{p}<\infty
$$

we denote by $L^{p}(\hat{\mu})$ while $0<p<\infty$, also $L^{\infty}(\hat{\mu})$ is the space of all $f$ for which there exists

$$
|f|_{\infty}:=\operatorname{ess}_{V(\hat{\mu}, *)} \sup |f|<\infty .
$$

In details we write $L^{p}\left(\mathcal{S}, \Phi, \hat{\mu}, \mathcal{A}_{v}\right)$ instead of $L^{p}(\hat{\mu})$, where

$$
V(\hat{\mu}, h):=V\left(\hat{v}, \mathcal{A}_{v}\right)
$$

with $h \geq 0$ non-negative $\hat{\mu}$-measurable function $h: \mathcal{A}_{v} \rightarrow[0, \infty), \quad \hat{v}(\mathrm{~d} y)=\hat{\mu}(\mathrm{d} y) h(y)$,

$$
\left|\hat{\mu}\left(h \chi_{U}\right)\right|^{2}:=\sum_{l}\left|\mu_{i_{0}, i_{l}}\left(h \chi_{U}\right)\right|^{2},
$$

since the function $h \chi_{U}$ is real-valued. A subset $W$ in
$\mathcal{S}$ we call $\mu$-zero-set, if $V^{*}(\hat{\mu}, W)=0$, where $V^{*}$ is the extension of the complete variation $V$ by the formula

$$
V^{*}(\hat{\mu}, A):=\inf _{\Phi \ni F \supset A} V(\hat{\mu}, F)
$$

for $A \subset \mathcal{S}$. An $\mathcal{A}_{v}$-valued measure $\hat{\lambda}$ on $\mathcal{S}$ we call absolutely continuous relative to $\hat{\mu}$, if $V^{*}(\hat{\lambda}, A)=0$ for each subset $A \subset \mathcal{S}$ with $V^{*}(\hat{\mu}, A)=0$. A measure $\hat{\mu}$ we call positive, if each $\mu_{m, n}$ is non-negative and there exist indices $m, n$ for which $\mu_{m, n}$ is positive (i.e. non-negative on its $\sigma$-algebra and positive on some elements of this $\sigma$-algebra).

For a reference we formulate the following non-commutative variants of Radon-Nikodym's theorem.

RNCD. Theorem. 1) If $(\mathcal{S}, \Phi, \hat{\mu})$ is a space with a $\sigma$-finite positive $\mathcal{A}_{v}$-valued measure $\hat{\mu}$, where $2 \leq v \leq$ 3 , also $\hat{\lambda}$ is an absolutely continuous relative to $\hat{\mu}$ bounded $\mathcal{A}_{v}$-valued measure defined on $\Phi$, then there exists a unique function $f \in L^{1}\left(\mathcal{S}, \Phi, \hat{\mu}, \mathcal{A}_{v}\right)$, such that $\hat{\lambda}(U)=\hat{\mu}\left(f \chi_{U}\right)$ for each $U \in \Phi$, moreover,

$$
V(\hat{\lambda}, \mathcal{S})=\|f\|_{1}:=\int_{\mathcal{S}}|f(y)| V(\hat{\mu}, \mathrm{d} y) .
$$

2) If $(\mathcal{S}, \Phi, \hat{\mu})$ is a space with bounded $\mathcal{A}_{v}$-valued measure $\hat{\mu}$, also $\hat{\lambda}$ is an absolutely continuous relative to $\hat{\mu} \mathcal{A}_{v}$-valued measure defined on $\Phi$, where $2 \leq v \leq 3$, then there exists a unique function $f \in L^{1}(\hat{\mu})$, such that $\hat{\lambda}(U)=\hat{\mu}\left(f \chi_{U}\right)$ for each $U \in \Phi$.

Proof. This follows from the corresponding RadonNikodym's theorems for $\boldsymbol{R}$-valued and $\boldsymbol{C}$-valued measures.

Recall the classical Radon-Nikodym theorem (III.10.2, 10.7 [19]). Let $(\mathcal{S}, \Phi, \mu)$ be a measure space with a $\sigma$-finite positive measure $\mu$ and let $v$ be a defined on $\Phi$ finite real-valued measure absolutely continuous relative to $\mu$, then there exists a unique function $f \in L^{1}(\mathcal{S}, \Phi, \mu, \boldsymbol{R})$ such that

$$
v(A)=\int_{A} f(y) \mu(\mathrm{d} y)
$$

for each $A \in \Phi$, moreover, $V(v, \mathcal{S})=\|f\|_{1}$. Let $(\mathcal{S}, \Phi, \mu)$ be a space with a finite complex-valued measure and let $v$ be a defined on $\Phi$ finite complex-valued measure absolutely continuous relative to $\mu$, then there exists a unique function $f \in L^{1}(\mathcal{S}, \Phi, \mu, C)$ such that

$$
v(A)=\int_{A} f(y) \mu(\mathrm{d} y)
$$

for each $A \in \Phi$.

In the quaternion skew field and the octonion algebra each equation of the form $a x=b$ or $x a=b$ for nonzero $a$ has the solution $x=a^{-1} b$ or $x=b a^{-1}$ respectively.

On the other hand, a measure $v_{n, k}$ is absolutely con- 
tinuous relative to $V(\hat{\mu})$ for each $n, k$, consequently, $v_{n, k}(\mathrm{~d} x)=h_{n, k}(x) V(\hat{\mu}, \mathrm{d} x)$ with

$h_{k, n} \in L^{1}(\mathcal{S}, \Phi, V(\hat{\mu}), \boldsymbol{R})$ and hence

$\hat{v}(\mathrm{~d} x)=h(x) V(\hat{\mu}, \mathrm{d} x)$, where $h \in L^{1}\left(\mathcal{S}, \Phi, V(\hat{\mu}), \mathcal{A}_{v}\right)$. Analogously, one gets $\hat{\mu}(\mathrm{d} x)=g(x) V(\hat{\mu}, \mathrm{d} x)$ with $g \in L^{1}\left(\mathcal{S}, \Phi, V(\hat{\mu}), \mathcal{A}_{v}\right)$. Thus $\hat{\mu}$ almost everywhere on $\mathcal{S}$ one obtains

$$
\hat{v}(\mathrm{~d} x)=\hat{\mu}(\mathrm{d} x) \cdot\left(\frac{1}{g(x)} h(x)\right) .
$$

\section{Continuation of the proof of Theorem 23.}

The $\mathcal{A}_{r}$-meta-invariance of $T$ implies, that the families of subalgebras $\Psi_{t}$ isomorphic with $\mathcal{A}_{r}$ exist, which are defined on vector sub-spaces ${ }^{t} X$ over $\Psi_{t}$ satisfying Conditions 2.9( $M 1-M 4)$. Therefore, we take restrictions $\left.T\right|_{t_{X}}$ and reduce the consideration to the case over $\mathcal{A}_{r}$ up to an isomorphism of the CayleyDickson algebras.

Recall that a mapping $f: U \rightarrow W$ of a topological space $U$ into a topological space $W$ is called closed if its graph $G(f)$ is a closed subset in $U \times W$, where

$G(f):=\{(x, f(x)): x \in U\}$. In view of criterion (14.1.1)

[15] a mapping $f: U \rightarrow W$ has a closed graph if and only if for each net ${ }_{a} x$ in $U$ converging to $x \in X$ and with $f\left({ }_{a} x\right) \rightarrow y$ the equality $y=f(x)$ is fulfilled.

We now prove that $f(T)$ is a closed operator. For each $n=1,2, \cdots$ we have $\hat{\boldsymbol{E}}\left(\delta_{n}\right) X \subset \mathcal{D}(f(T))$ and $\lim _{n} \hat{\boldsymbol{E}}\left(\delta_{n}\right) x=x$ for each $x \in X$, consequently, a domain of definition $\mathcal{D}(f(T))$ of $f(T)$ is everywhere dense in $X$. Take a sequence $\left\{{ }_{n} x: n\right\} \subset \mathcal{D}(f(T))$ so that $\lim _{n n} x=x$ and $\lim _{n} f(T){ }_{n} x=y$. Then for each natural number $m$ we get the equalities:

$$
\begin{aligned}
f_{m}(T) x & =\lim _{n} f_{m}(T)_{n} x \\
& =\lim _{n} \hat{\boldsymbol{E}}\left(\delta_{m}\right) f(T)_{n} x=\hat{\boldsymbol{E}}\left(\delta_{m}\right) y .
\end{aligned}
$$

Thus

$$
y=\lim _{m} \hat{\boldsymbol{E}}\left(\delta_{m}\right) y=\lim _{m} f_{m}(T) x=f(T) x,
$$

hence $x \in \mathcal{D}(f(T))$ and the operator $f(T)$ is closed.

Let $x \in \mathcal{D}(f(T)), \quad y \in X$. Due to $R N C D(i i)$ for the variation $\hat{v}(\delta):=V\left(\hat{\mu}_{x, y}, \delta\right)$ of the measure $\hat{\mu}_{x, y}$ there exists a Borel measurable function $\phi$, such that $\hat{v}(\delta):=\hat{\mu}_{x, y}\left(\phi \chi_{\delta}\right)=\left\langle\hat{\boldsymbol{E}}\left(\phi \chi_{\delta}\right) x ; y\right\rangle$ for each $\delta \in \mathcal{B}(\boldsymbol{R})$. From $R N C D(i)$ it follows that $|\phi(z)|=1 \quad \hat{v}$-almost everywhere. Consider $f^{1}(z):=|f(z)| \phi(z)$, then due to (a) $\mathcal{D}\left(f^{1}(T)\right)=\mathcal{D}(f(T))$ and

$$
\begin{aligned}
\left\langle f^{1}(T) x ; y\right\rangle & =\lim _{n} \int_{\delta_{n}}|f(z)|\langle\hat{\boldsymbol{E}}(\mathrm{d} z) \phi(z) x ; y\rangle \\
& =\int_{-\infty}^{\infty}|f(z)| \hat{v}(\mathrm{~d} z),
\end{aligned}
$$

since $\boldsymbol{R}$ is the center of the Cayley-Dickson algebra and $0 \leq|f(z)| \in \boldsymbol{R}$ for each $z$. Therefore,

$$
\begin{aligned}
\langle f(T) x ; y\rangle & =\lim _{n} \int_{\delta_{n}}\langle\hat{\boldsymbol{E}}(\mathrm{d} z) \cdot f(z) x ; y\rangle \\
& =\int_{-\infty}^{\infty}\langle\hat{\boldsymbol{E}}(\mathrm{d} z) \cdot f(z) x ; y\rangle
\end{aligned}
$$

and from this it follows b).

d) From $\hat{\boldsymbol{E}}_{\mathcal{S}}^{*}=(-1)^{\eta(\mathcal{S})} \hat{\boldsymbol{E}}_{\mathcal{S}}$ for each $\mathcal{S}=c s$,

$0 \neq c \in \boldsymbol{R}, \quad s \in\left\{i_{0}, \cdots, i_{m}, \cdots\right\}$, where $\eta\left(i_{0}\right)=0$ and $\eta\left(i_{j}\right)=1$ for each $j \geq 1$, it follows, that $E \cdot \tilde{f}=E^{*} \cdot f$. Take $x, y \in \mathcal{D}(\tilde{f}(T))=\mathcal{D}(f(T))$, then

$$
\langle\tilde{f}(T) x ; y\rangle=\int_{-\infty}^{\infty}\langle\hat{\boldsymbol{E}}(\mathrm{d} z) \cdot \tilde{f}(z) x ; y\rangle=\langle x ; f(T) y\rangle,
$$

consequently, $\tilde{f}(T) \subset f(T)^{*}$. If $y \in \mathcal{D}\left(f(T)^{*}\right)$, then for each $x \in X$ and $\tau \in \boldsymbol{N}$ one gets that

$$
\begin{aligned}
\left\langle x ; \tilde{f}_{\tau}(T) y\right\rangle & =\left\langle f_{\tau}(T) x ; y\right\rangle=\left\langle f(T) \hat{\boldsymbol{E}}\left(\delta_{\tau}\right) x ; y\right\rangle \\
& =\left\langle x ; \hat{\boldsymbol{E}}\left(\delta_{\tau}\right) f(T)^{*} y\right\rangle,
\end{aligned}
$$

consequently, $\tilde{f}_{\tau}(T) y:=\hat{\boldsymbol{E}}\left(\delta_{\tau}\right) f(T)^{*} y$ converges to $f(T)^{*} y$ for $\tau \rightarrow \infty$ and inevitably $y \in \mathcal{D}(\tilde{f}(T))$.

e) Due to Theorem 2.24 [18] the statement $(e)$ follows from the fact that ${ }_{\tau} \hat{\boldsymbol{E}}(\delta):=\hat{\boldsymbol{E}}\left(\delta_{\tau} \cap \delta\right)$ is the decomposition of the unity for the bounded restriction $\left.T\right|_{X_{\tau}}$, where $X_{\tau}:=\hat{\boldsymbol{E}}\left(\delta_{\tau}\right) X$. On the other hand, $R(q ; T)\left[(q I-T) \hat{\boldsymbol{E}}\left(\delta_{\tau}\right)\right]=\hat{\boldsymbol{E}}\left(\delta_{\tau}\right)$ for each $\tau \in \boldsymbol{N}$ and $q \in \rho(T)$. Clearly $\left.R(q ; T)\right|_{X_{\tau}}=R\left(q ;\left.T\right|_{X_{\tau}}\right)$. Therefore,

$$
\begin{aligned}
R(q ; T) x & =\lim _{\tau \rightarrow \infty} R(q ; T) \hat{\boldsymbol{E}}\left(\delta_{\tau}\right) x \\
& =\lim _{\tau} \int_{\delta_{\tau}} \hat{\boldsymbol{E}}(\mathrm{d} b) \cdot \frac{1}{q-b} x=\int_{-\infty}^{\infty} \hat{\boldsymbol{E}}(\mathrm{d} b) \cdot \frac{1}{q-b} x
\end{aligned}
$$

that finishes the proof.

24) Theorem. A bounded normal $\mathcal{A}_{r}$-meta-invariant operator $T$ on a Hilbert space over the Cayley-Dickson algebra $\mathcal{A}_{v}, \quad 2 \leq v, \quad 1 \leq r \leq \min (3, v)$, is unitary, Hermitian or positive if and only if $\sigma(T)$ is contained in $\mathcal{S}_{v}:=\left\{z \in \mathcal{A}_{v}:|z|=1\right\}, \quad \boldsymbol{R}$ or $[0, \infty)$ respectively.

Proof. Due to Theorem 2.24 [18] the equality $T^{*} T=T T^{*}=I$ is equivalent to $z \tilde{z}=1$ for each $z \in \sigma(T)$. If $\sigma(T) \subset[0, \infty)$, then

$$
\langle T x ; x\rangle=\int_{\sigma(T)}\langle\hat{\boldsymbol{E}}(\mathrm{d} z) \cdot z x ; x\rangle \geq 0
$$

for each $x \in X$. Applying Theorems 4 and 12 we get the statement of this theorem.

25) Definition. A family $\{T(t): 0 \leq t \in \boldsymbol{R}\}$ of bounded quasi-linear operators in a vector space $X$ over the Cayley-Dickson algebra $\mathcal{A}_{v}, 2 \leq v$, is called a strongly continuous semigroup, if

a) $T(t+q)=T(t) T(q)$ for each $t, q \geq 0$; 
b) $T(0)=I$;

c) $T(t) x$ is a continuous function by $t \in[0, \infty)$ for each $x \in X$;

For $t>0$ let an operator $\mathcal{A}_{t}$ be defined by the equality

d) $\mathcal{A}_{t} x=\frac{T(t) x-x}{t}$ for each $x \in X$.

Denote by $\mathcal{D}(A)$ the set of all vectors $x \in X$ for

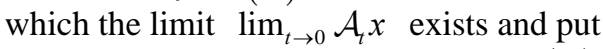

e) $A x:=\lim _{t \downarrow 0} \mathcal{A}_{t} x$ for each $x \in \mathcal{D}(A)$. This operator $A$ is called an infinitesimal generator of the oneparameter semigroup $U(t)$.

26) Theorem. For each strongly continuous semigroup $\{U(t): 0 \leq t \in \boldsymbol{R}\}$ of unitary quasi-linear $\mathcal{A}_{r}$ meta-invariant operators in a Hilbert space $X$ over the Cayley-Dickson algebra $\mathcal{A}_{v}, 2 \leq v<\infty$,

$1 \leq r \leq \min (3, v)$, there exists a unique self-adjoint $\mathcal{A}_{r}$ meta-invariant quasi-linear operator $B$ in $X$ so that

1) $\langle U(t) x ; y\rangle=\int_{-\infty}^{\infty}\langle\hat{\boldsymbol{E}}(\mathrm{d} b) \cdot \exp (t M(b) b) x ; y\rangle$,

2) $\langle B x, y\rangle=\int_{-\infty}^{\infty} b\langle\hat{\boldsymbol{E}}(\mathrm{d} b) x ; y\rangle$

for each $x, y \in \mathcal{D}(B)$, where $\hat{\boldsymbol{E}}$ is an $\mathcal{A}_{v}$ graded projection valued measure, $M(b)$ is a Borel function from $\boldsymbol{R}$ into $\mathcal{S}_{v}:=\left\{z \in \mathcal{A}_{v}:|z|=1, \operatorname{Re}(z)=0\right\}$.

If additionally $r=1$ and $U(t)$ is either left or right $\mathcal{A}_{v}$-linear operator for each $t$, then there exists a marked purely imaginary Cayley-Dickson number $M \in \mathcal{S}_{v}$ such that

3) $U(t)=\exp (t M B)$ for each $t \geq 0$.

Proof. For a marked $t>0$ this was partially demonstrated in Section 10. Then we demonstrate it relative to the parameter $t$. Extent a semigroup $U(t)$ for $t \geq 0$ to a group $U(t)$ for each $t \in \boldsymbol{R}$ putting $U(-t)=U^{*}(t)$.

Consider the space $C_{0}^{\infty}(\boldsymbol{R}, \boldsymbol{R})$ of all infinitely differentiable functions $f: \boldsymbol{R} \rightarrow \boldsymbol{R}$ with compact support. For each $x \in X$ and $f \in C_{0}^{\infty}(\boldsymbol{R}, \boldsymbol{R})$ consider the integral

4) $x^{f}:=\int_{-\infty}^{\infty} f(t) U(t) x \mathrm{~d} t$.

Since the group $U(t)$ is strongly continuous, this integral can be considered as the Riemann integral. Take the $\mathcal{A}_{v}$-vector space

$$
Y:=\operatorname{span}_{\mathcal{A}_{v}}\left\{x^{f}: x \in X, f \in C_{0}^{\infty}(\boldsymbol{R}, \boldsymbol{R})\right\} .
$$

Then we choose a function $w(b) \in C_{0}^{\infty}(\boldsymbol{R}, \boldsymbol{R})$ with the support

$$
\begin{aligned}
\text { supp } w & :=\operatorname{cl}\{b: w(b) \neq 0\} \subset(-1,1) \\
& :=\{b \in \boldsymbol{R}:-1<b<1\}
\end{aligned}
$$

and $w(b) \geq 0$ for each $b$ and $w(b)>0$ on some interval $\left(-\epsilon_{0}, \epsilon_{0}\right)$ and

$$
\int_{-\infty}^{\infty} w(b) \mathrm{d} b=1
$$

where $\epsilon_{0}>0$. Then we put $w_{u}(b):=\frac{w(b / u)}{u}$ for $u>0$. It can be lightly seen that

$$
\text { 5) } \begin{aligned}
\left\|x^{w_{u}}-x\right\| & =\left\|\int_{-\infty}^{\infty} w_{u}(t)(U(t) x-x) \mathrm{d} t\right\| \\
& \leq\left[\int_{-\infty}^{\infty} w_{u}(t) \mathrm{d} t\right] \sup _{t \in[-u, u]}\|U(t) x-x\| .
\end{aligned}
$$

The one-parameter group $U(t)$ is strongly continuous, consequently, the $\mathcal{A}_{v}$-vector space $Y$ is everywhere dense in $X$. For $x^{f} \in Y$ we deduce

$$
\text { 6) } \begin{aligned}
\frac{U(q)-I}{q} x^{f} & =\int_{-\infty}^{\infty} f(t) \frac{U(q+t)-U(t)}{q} x \mathrm{~d} t \\
& =\int_{-\infty}^{\infty} \frac{f(\tau-q)-f(\tau)}{q} U(\tau) x \mathrm{~d} \tau,
\end{aligned}
$$

consequently,

$$
\lim _{q \rightarrow 0} \frac{U(q)-I}{q} x^{f}=-\int_{-\infty}^{\infty} f^{\prime}(\tau) U(\tau) x \mathrm{~d} \tau=x^{-f^{\prime}},
$$

since the function $[f(q-\tau)-f(\tau)] / q$ uniformly converges to $-f^{\prime}(\tau)$ on $\boldsymbol{R}$ when $q$ tends to zero. Then we put $Q x^{f}:=x^{-f^{\prime}}$. From the definition of $Q$ we get that $Q U(t) x^{f}=U(t) Q x^{f}$ for each $x^{f} \in Y$ and $t \in \boldsymbol{R}$, since $U(t)$ is the one-parameter group and $\boldsymbol{R}$ is the center of the Cayley-Dickson algebra $\mathcal{A}_{v}$, which implies

$$
U(t) \frac{U(q)-I}{q}=\frac{U(q+t)-U(t)}{q}=\frac{U(q)-I}{q} U(t) .
$$

Then the equalities are satisfied:

$$
\begin{aligned}
& \left\langle Q^{*} Q x^{f} ; x^{g}\right\rangle \\
& =\left\langle Q x^{f} ; Q x^{g}\right\rangle=\lim _{q \rightarrow 0, t \rightarrow 0}\left\langle\frac{U(q)-I}{q} x^{f} ; \frac{U(t)-I}{t} x^{g}\right\rangle \\
& =\left\langle x^{-f^{\prime}} ; x^{-g^{\prime}}\right\rangle=\left\langle Q Q^{*} x^{f} ; x^{g}\right\rangle,
\end{aligned}
$$

since $U(-t)=U^{*}(t)$ and

$$
\left[\frac{U(q)-I}{q}\right]^{*}=\frac{U(-q)-I}{q}=\frac{I-U(-q)}{-q}
$$

and $U(t)$ is the one-parameter group, consequently, $Q$ is the normal $\mathcal{A}_{r}$-meta-invariant operator. We have that 


$$
\begin{aligned}
& \left\langle\left(Q+Q^{*}\right) x^{f} ; x^{g}\right\rangle \\
& =\lim _{q \rightarrow 0}\left\langle\left[\frac{U(q)-I}{q}+\frac{U(-q)-I}{q}\right] x^{f} ; x^{g}\right\rangle \\
& =\lim _{q \rightarrow 0}\left\langle\frac{U(q)-I}{q} x^{f} ; x^{g}\right\rangle-\lim _{q \rightarrow 0}\left\langle\frac{U(-q)-I}{-q} x^{f} ; x^{g}\right\rangle=0,
\end{aligned}
$$

hence $Q=-Q^{*}$ and the operator $Q$ is skew-adjoint. Then $\left(\begin{array}{cc}0 & Q \\ -Q & 0\end{array}\right)$ is self-adjoint on $Y^{2}$. By Theorem 2.24 [18] it has the $\mathcal{A}_{v}$ graded projection valued measure $\hat{\boldsymbol{F}}$ and

$$
\begin{aligned}
\langle Q x ; y\rangle & =\int_{A_{r}}\langle\hat{\boldsymbol{F}}(\mathrm{d} z) \cdot z x ; y\rangle=-\left\langle Q^{*} x ; y\right\rangle \\
& =-\int_{A_{r}}\left\langle\hat{\boldsymbol{F}}(\mathrm{d} z) \cdot z^{*} x ; y\right\rangle
\end{aligned}
$$

for each $x, y \in Y$, since $C \subset \mathcal{A}_{r}$, consequently, $\hat{\boldsymbol{F}}\left(\mathrm{d} z^{*}\right)=-\hat{\boldsymbol{F}}(\mathrm{d} z)$. Take ${ }_{b} \hat{\boldsymbol{F}}:=\int_{\mathcal{B}\left(A_{r}, 0, b\right)} \hat{\boldsymbol{F}}(\mathrm{d} z)$ and ${ }_{b} X:={ }_{b} \hat{\boldsymbol{F}} X$ for $0<b<\infty$, where $B\left(\mathcal{A}_{r}, 0, b\right)$ denotes as usually the closed ball in $\mathcal{A}_{r}$ with center at zero and of radius $b$. Then

$$
{ }_{b} Q:=\int_{B\left(\mathcal{A}_{r}, 0, b\right)} \hat{\boldsymbol{F}}(\mathrm{d} z) \cdot z
$$

is the bounded operator on ${ }_{b} X,{ }_{b} Q \in L_{q}\left({ }_{b} X\right)$ up to an isomorphism of $\mathcal{A}_{v}$ Hilbert spaces. In view of Theorems 3.4 [18], 4, 9, 12 above the von Neumann algebra $\mathrm{cl}\left[\operatorname{alg}_{\mathcal{A}_{r}}\left({ }_{b} Q,{ }_{b} Q^{*}, I\right)\right]$ over $\mathcal{A}_{r}$ generated by ${ }_{b} Q,{ }_{b} Q^{*}$ and $I$ is-isomorphic with $C\left(\Lambda_{b}, A_{r}\right)$ for an extremely disconnected compact topological space $\Lambda_{b}$. Then $\theta\left({ }_{b} Q\right)={ }_{b} f$ is a skew-symmetric function $\left[{ }_{b} f(s)\right]^{*}={ }_{b} f(s)$ for each $s \in \Lambda_{b}$ and hence has the form ${ }_{b} f(s)={ }_{b} \phi(s){ }_{b} N(s)$ with ${ }_{b} \phi(s) \in \boldsymbol{R}$ and ${ }_{b} N(s) \in \mathcal{S}_{r}$ for each $s \in \Lambda_{b}$, where ${ }_{b} \phi \in C\left(\Lambda_{b}, \boldsymbol{R}\right)$ and ${ }_{b} N \in C\left(\Lambda_{b}, \mathcal{A}_{r}\right)$,

$$
\theta: c l\left[a \lg _{\mathcal{A}_{r}}\left({ }_{b} Q,{ }_{b} Q^{*}, I\right)\right] \rightarrow C\left(\Lambda_{b}, \mathcal{A}_{r}\right)
$$

denotes a ${ }^{*}$-isomorphism. We have that $\hat{\boldsymbol{F}}(\alpha) \hat{\boldsymbol{F}}(\beta)=\hat{\boldsymbol{F}}(\alpha \cap \beta)$ for each Borel subsets $\alpha$ and $\beta$ contained in $\mathcal{A}_{r}$. Therefore, up to $\mathrm{a}^{*}$-isomorphism of $C^{*}$-algebras the aforementioned functions and algebras can be chosen consistent: $\Lambda_{b} \subset \Lambda_{c},\left.{ }_{c} \phi\right|_{\Lambda_{b}}={ }_{b} \phi$ and $\left.{ }_{c} N\right|_{\Lambda_{b}}={ }_{b} N$ for each $0<b<c<\infty$.

Then we get $\theta^{-1}\left({ }_{b} \phi\right)=:{ }_{b} A$ is the self-adjoint $\mathcal{A}_{r}$ meta-invariant operator with an $\mathcal{A}_{v}$-graded projection valued measure $\hat{\boldsymbol{E}}$ on $\mathcal{B}([-b, b])$ so that

$$
\left\langle{ }_{b} A x ; y\right\rangle=\int_{-b}^{b} \tau\langle\hat{\boldsymbol{E}}(\mathrm{d} \tau) x ; y\rangle
$$

for each $x, y \in{ }_{b} X$. Therefore,

$$
\left\langle_{b} Q x ; y\right\rangle=\int_{-b}^{b} \tau\langle\hat{\boldsymbol{E}}(\mathrm{d} \tau) \cdot M(\tau) x ; y\rangle,
$$

where $M(\tau)$ is a Borel function with values in $\mathcal{S}_{v}$ (see Theorems 15, 16).

We now take a consistent family of $\mathcal{A}_{v}$-graded projection valued measures $\hat{\boldsymbol{E}}$ on $\bigcup_{b \in N}[-b, b]=\boldsymbol{R}$ and put

$$
B=\int_{-\infty}^{\infty} \tau \hat{\boldsymbol{E}}(\mathrm{d} \tau) .
$$

This operator is self-adjoint with a domain of definition $\mathcal{D}(B)$ given by Theorem 21. Consider the operator

$$
\langle V(t) x ; y\rangle=\int_{-\infty}^{\infty}\langle\hat{\boldsymbol{E}}(\mathrm{d} \tau) \cdot \exp (t M(\tau) \tau) x ; y\rangle,
$$

$x, y \in X, t \in \boldsymbol{R}$. Since $|\exp (t M(\tau) \tau)|=1$ for each $t, \tau \in \boldsymbol{R}$ and $\hat{\boldsymbol{E}}$ is the $\mathcal{A}_{v}$-graded projection valued measure on $\mathcal{B}(\boldsymbol{R})$, operators $V(t)$ are well defined. Their family by $t \in \boldsymbol{R}$ forms the one-parameter group of normal $\mathcal{A}_{r}$-meta-invariant unitary operators with a domain given by Theorem 24. Indeed, the projection valued measure $\hat{\boldsymbol{E}}$ is $\mathcal{A}_{v}$ graded and

$$
[\exp (t M(\tau) \tau)]^{*}=\exp (-t M(\tau) \tau)
$$

for each $t, \tau \in \boldsymbol{R}$, since $M^{*}(\tau)=-M(\tau)$ for every $\tau \in \boldsymbol{R}$.

It remains to show that $V(t)=U(t)$ for each $t \in \boldsymbol{R}$. There is the inclusion $Y \subset \mathcal{D}(B)$. Let $x \in Y$, then $V(t) x \in \mathcal{D}(B)$ and $\frac{\mathrm{d} V(t)}{\mathrm{d} t}=Q V(t) x$. On the other hand, $U(t) x \in \mathcal{D}(B)$ for each $t$. We consider $\omega(t)=U(t) x-V(t) x$ and get

$$
\frac{\mathrm{d} \omega(t)}{\mathrm{d} t}=Q U(t) x-Q V(t) x=Q \omega(t),
$$

consequently,

$$
\frac{\mathrm{d}\|\omega(t)\|^{2}}{\mathrm{~d} t}=\langle Q \omega(t) ; \omega(t)\rangle+\langle\omega(t) ; Q \omega(t)\rangle=0,
$$

since the operator $Q$ is skew-symmetric. But $\omega(0)=0$, consequently, $\omega(t)=0$ for each $t \in \boldsymbol{R}$. Thus the equality $U(t) x=V(t) x$ is fulfilled for each $t \in \boldsymbol{R}$ and $x \in Y$. The $\mathcal{A}_{v}$ vector subspace $Y$ is everywhere dense in $X$, consequently, $U(t)=V(t)$ for each $t \in \boldsymbol{R}$.

When the one-parameter group $U(t)$ satisfies additional conditions either left or right $\mathcal{A}_{v}$-linearity and $r=1$, then there exists a marked purely imaginary Cayley-Dickson number $M \in \mathcal{S}_{v}$ such that $U(t)=\exp (t M B)$ for each $t \geq 0$ due to Theorem 10 
and the proof above, since in this case $N(s)=N$ and $M(\tau)=M$ are constants in accordance with Conditions $25(i-v)$.

27) Remark. Another way of the preceding theorem proof in the particular case $r=1$ and either left or right $\mathcal{A}_{v}$ linear operators $U(t)$ for each $t$ is the following.

If $\{T(t): 0 \leq t\}$ is a semigroup continuous in the operator norm topology (see also the complex case in Theorem VIII.1.2 [19]), then there exists a bounded $\mathcal{A}_{1}$-meta-invariant either left or right $\mathcal{A}_{v}$-linear operator $A$ on $X$ such that $T(t)=\exp (t A)$ for each $t \geq 0$. If $\operatorname{Re}(z):=(z+\tilde{z}) / 2>|A|$, then

$$
|\exp (-t(z I-A))| \leq \exp (t(|A|-\operatorname{Re}(z))) \rightarrow 0
$$

for $t \rightarrow \infty$. For such $z \in \mathcal{A}_{v}$ due to the Lebesgue theorem:

$$
(z I-A) \int_{0}^{\infty} \exp (-t(z I-A)) \mathrm{d} t=I,
$$

also by Lemma 2.8 there exists

$$
R(z ; A)=\int_{0}^{\infty} \exp (-t(z I-A)) \mathrm{d} t .
$$

For each $\epsilon>0$ let $\mathcal{A}_{\epsilon} x:=(T(\epsilon) x-x) / \epsilon$, where $x \in X$, for which there exists $\lim _{0<\epsilon \rightarrow 0} \mathcal{A}_{\epsilon} x$, the set of all such $x$ we denote by $\boldsymbol{D}(A)$. Evidently, $\boldsymbol{D}(A)$ is the $\mathcal{A}_{v}$ vector subspace in $X$. Take some infinitesimal quasilinear operator $A x:=\lim _{0<\epsilon \rightarrow 0} \mathcal{A}_{\epsilon} x$. Considering $\mathcal{A}_{v}$ as the Banach space over $\boldsymbol{R}$, we get the analogs of Lemmas 3, 4, 7, Corollaries 5, 9 and Theorem 10 from $\S$ VIII.1 [19], moreover, $\mathcal{D}(A)$ is dense in $X$, also $A$ is the closed quasi-linear $\mathcal{A}_{1}$-meta-invariant operator on $\boldsymbol{D}(A)$. Let $w_{0}:=\lim _{t \rightarrow \infty} \ln (|T(t)|) / t$ and $z \in \mathcal{A}_{v}$ with $\operatorname{Re}(z)>w_{0}$. For each $w_{0}<\delta<\operatorname{Re}(z)$ due to Corollary VIII.1.5 [19] a constant $C>0$ exists such that $|T(t)| \leq C \exp (\delta t)$ for each $t \geq 0$. Then there exists $R(z) x:=\int_{0}^{\infty} \exp (-t(z I-A)) x \mathrm{~d} t$ for each $x \in X$ and $\operatorname{Re}(z)>w_{0}$, consequently, $R(z) x \in \boldsymbol{D}(A)$.

Let $T_{z}$ be a quasi-linear operator corresponding to $\left(z^{-1} I\right) A$ instead of $T$ for $A$, where $0 \neq z \in \mathcal{A}_{v}$, moreover, $\boldsymbol{D}(A)=\left(\boldsymbol{D}\left(z^{-1} I\right) A\right)$. Then

$$
\begin{aligned}
& \left(z^{-1} I\right) A \int_{0}^{\infty} \exp \left(-t\left(I-\left(z^{-1} I\right) A\right)\right) x \mathrm{~d} t \\
& =\int_{0}^{\infty} \exp \left(-t\left(I-\left(z^{-1} I\right) A\right) z^{-1}\right) A x \mathrm{~d} t,
\end{aligned}
$$

consequently, $R(z)(z I-A) x=x$ for each $x \in \boldsymbol{D}(A)$ and $R(z)=R(z ; A)$. Thus,

$$
R(z ; A) x=\int_{0}^{\infty} \exp (-t(z I-A)) x \mathrm{~d} t
$$

for each $z \in \rho(A)$ and $x \in X$.

From \$2.39 [18] it follows, that for the quasi-linear operator $A$ the quasi-linear operator $B$ exists such that $A=M B$, where $M \in \mathcal{A}_{v},|M|=1, \operatorname{Re}(M)=0$. In view of the identities $U(t) U(t)^{*}=U(t)^{*} U(t)=I$ we get that $A$ commutes with $A^{*}$ and $\exp \left(t\left(A+A^{*}\right)\right)=I$. From the equality $R(z ; B)^{*}=R(\tilde{z}, B)$ it follows, that we can choose $B=B^{*}$. If $\hat{E}$ is the decomposition of the unity for $B$ and $V(t):=\exp (M t B)$, then by Theorem 2.30 [18] we have

$$
\langle V(t) x ; y\rangle=\int_{-\infty}^{\infty}\langle\hat{E}(\mathrm{~d} z) \cdot \exp (M t z) x ; y\rangle,
$$

then due to the Fubini theorem one gets the equality

$$
\begin{aligned}
& \int_{0}^{\infty}\langle V(t) \cdot \exp (-b t) x ; y\rangle \mathrm{d} t \\
& =\int_{0}^{\infty} \int_{-\infty}^{\infty}\langle\hat{E}(\mathrm{~d} z) \cdot \exp (-(b-M z) t) x ; y\rangle \mathrm{d} t \\
& =\int_{-\infty}^{\infty}\left\langle\hat{E}(\mathrm{~d} z) \cdot(b-M z)^{-1} x ; y\right\rangle=R(b ; M B) x ; y
\end{aligned}
$$

for each $b \in \boldsymbol{R} \oplus \boldsymbol{R} M$ with $\operatorname{Re}(b)>0$. Therefore, we infer

$$
\int_{0}^{\infty}\langle V(t) \cdot \exp (-b t) x ; y\rangle \mathrm{d} t=\int_{0}^{\infty}\langle U(t) \cdot \exp (-b t) x ; y\rangle \mathrm{d} t
$$

for $\operatorname{Re}(b)>0$. Due to Lemma VIII.1.15 the equality

$$
\langle V(t) \cdot \exp (-\epsilon t) x ; y\rangle=\langle U(t) \cdot \exp (-\epsilon t) x ; y\rangle
$$

is fulfilled for each $t \geq 0$ and for every $b \in \boldsymbol{R} \oplus \boldsymbol{R} M$ with $\operatorname{Re}(b)>0$, consequently, $U(t)=V(t)$.

28) Definitions. A topological $\mathcal{A}_{v}$-vector space $X$ is called locally convex, if it has a base of open neighborhoods of zero consisting of $\mathcal{A}_{v}$-convex open subsets $U$, that is $(a x) b+c(y g) \in U$ for each $a, b, c, g \in \mathcal{A}_{v}$ with $|a||b|+|c||g| \leq 1$ and every $x, y \in U$. Let $X$ be an $\mathcal{A}_{v}$-vector locally convex space. Consider left and right and two sided $\mathcal{A}_{v}$-vector spans of the family of vectors $\left\{\eta^{a}: a \in A\right\}$, where

$$
\begin{aligned}
& \operatorname{span}_{\mathcal{A}_{v}}^{l}\left\{\eta^{a}: a \in \boldsymbol{A}\right\} \\
& :=\left\{z \in X: z=\sum_{g_{a}^{1}, \cdots, g_{a}^{k} \in \mathcal{A}_{v} ; a \in \mathbf{A} ; k \in N} g_{a}^{1}\left(\cdots\left(g_{a}^{k} \eta^{a}\right) \cdots\right)\right\} ; \\
& \operatorname{span}_{\mathcal{A}_{v}}^{r}\left\{\eta^{a}: a \in \boldsymbol{A}\right\} \\
& :=\left\{z \in X: z=\sum_{g_{a}^{1}, \cdots, g_{a}^{k} \in \mathcal{A}_{v} ; a \in \mathbf{A} ; k \in N}\left(\cdots\left(\eta^{a} g_{a}^{1}\right) \cdots\right) g_{a}^{k}\right\} ; \\
& \operatorname{span}_{\mathcal{A}_{v}}\left\{\eta^{a}: a \in \boldsymbol{A}\right\} \\
& :=\left\{z \in X: z=\sum_{g_{a}, r_{a} \in \mathcal{A}_{v} ; a \in \mathbf{A}}\left\{g_{a} \eta^{a} r_{a}\right\}_{q(3)}\right\},
\end{aligned}
$$

where $q(3)$ is a vector prescribing an order of the 
multiplication in the curled brackets.

29) Lemma. In the notation of $\S 28$

$$
\begin{aligned}
\operatorname{span}_{\mathcal{A}_{v}}^{l}\left\{\eta^{a}: a \in \boldsymbol{A}\right\} & =\operatorname{span}_{\mathcal{A}_{v}}^{r}\left\{\eta^{a}: a \in \boldsymbol{A}\right\} \\
& =\operatorname{span}_{\mathcal{A}_{v}}\left\{\eta^{a}: a \in \boldsymbol{A}\right\} .
\end{aligned}
$$

Proof. Due to the continuity of the addition and multiplication on scalars of vectors in $X$ and using the convergence of nets in $X$, it is sufficient to prove the statement of this lemma for a finite set $\boldsymbol{A}$. Then the space

$Y:=\operatorname{span}_{\mathcal{A}_{v}}\left\{\eta^{a}: a \in \boldsymbol{A}\right\}$ is finite dimensional over $\mathcal{A}_{v}$ and evidently left and right $\mathcal{A}_{\text {, }}$-vector spans are contained in it. Then in $Y$ it can be chosen a basis over $\mathcal{A}_{v}$ and each vector can be written in the form $\eta^{a}=\left\{\eta_{1}^{a}, \cdots, \eta_{n}^{a}\right\}$, where $n \in N, \eta_{s}^{a} \in \mathcal{A}_{v}$.

Each $z \in \mathcal{A}_{v}$ can be written in the polar form $z=|z| \mathrm{e}^{t M}$, where $t \in \boldsymbol{R}, \quad t$ is a real parameter, and $M$ is a purely imaginary Cayley-Dickson number, $M \in \mathcal{A}_{v}$ of unit absolute value $|M|=1$ and $\operatorname{Re}(M)=0$. Hence $(z \xi) \zeta=\zeta(\xi z)=\alpha|z| \zeta$ for each $\xi=\alpha \mathrm{e}^{-t M}$ with real parameter $\alpha, \alpha \in \boldsymbol{R}$, and a Cayley-Dickson number $\zeta \in \mathcal{A}_{v}$, since the real field $\boldsymbol{R}$ is the center of the Cayley-Dickson algebra $\mathcal{A}_{v}$.

On the other hand, $X=X_{0} i_{0} \oplus X_{1} i_{1} \oplus \cdots \oplus X_{m} i_{m} \oplus \cdots$, where $X_{0}, \cdots, X_{m}, \cdots$ are pairwise isomorphic $\boldsymbol{R}$-linear locally convex spaces. Therefore, we have

$$
\begin{aligned}
& \operatorname{span}_{\mathcal{A}_{v}}^{l}\left\{\eta^{a}: a \in \boldsymbol{A}\right\} \cap \operatorname{span}_{\mathcal{A}_{v}}^{r}\left\{\eta^{a}: a \in \boldsymbol{A}\right\} \\
& \supset \operatorname{span}_{\mathcal{A}_{v}}\left\{\eta^{a}: a \in \boldsymbol{A}\right\},
\end{aligned}
$$

that together with the inclusion

$$
\begin{aligned}
& \operatorname{span}_{\mathcal{A}_{v}}^{r}\left\{\eta^{a}: a \in \boldsymbol{A}\right\} \cup \operatorname{span}_{\mathcal{A}_{v}}^{l}\left\{\eta^{a}: a \in \boldsymbol{A}\right\} \\
& \subset \operatorname{span}_{\mathcal{A}_{v}}\left\{\eta^{a}: a \in \boldsymbol{A}\right\}
\end{aligned}
$$

proved above leads to the statement of this lemma.

30) Lemma. Let $X$ be a Hilbert space over the CayleyDickson algebra $\mathcal{A}_{v}$, also $X_{R}$ be the same space considered over the real field $\boldsymbol{R}$. A vector $x \in X$ is orthogonal to an $\mathcal{A}_{v}$-vector subspace $Y$ in $X$ relative to the $\mathcal{A}_{v}$-valued scalar product in $X$ if and only if $x$ is orthogonal to $Y_{R}$ relative to the scalar product in $X_{R}$. The space $X$ is isomorphic to the standard Hilbert space $l_{2}\left(\alpha, \mathcal{A}_{v}\right)$ over $\mathcal{A}_{v}$ of converging relative to the norm sequences or nets $\eta=\left\{\eta^{a}: a \in \alpha\right\}$ with the scalar product $\langle\eta ; w\rangle:=\sum_{a} \tilde{\eta}^{a} w_{a}$, moreover,

$d(X)=\operatorname{card}(\alpha) \aleph_{0} 2^{\operatorname{card}(v)}$, where $\operatorname{card}(\alpha)$ is the cardinality of a set $\alpha, \aleph_{0}=\operatorname{card}(N), d(X)$ denotes the topological density of $X$.

Proof. Due to Lemma 29 and by the transfinite induction in $Y$, an $\mathcal{A}_{v}$-vector independent system of vectors $\left\{\eta^{a}: a \in \boldsymbol{A}\right\}$ exists, such that $\operatorname{span}_{\mathcal{A}_{v}}^{r}\left\{\eta^{a}: a \in \boldsymbol{A}\right\}$ is everywhere dense in $Y$. In another words in $Y$, a Hamel basis over $\mathcal{A}_{v}$ exists. A vector $x$ by the definition is orthogonal to $Y$ if and only if $\langle\eta ; x\rangle=0$ for each $\eta \in Y$, that is equivalent to $\left\langle\eta^{a} ; x\right\rangle=0$ for each $a \in \boldsymbol{A}$. The space $X$ is isomorphic to the direct sum

$X_{0} \oplus X_{1} i_{1} \oplus \cdots \oplus X_{m} i_{m} \oplus \cdots$, where $X_{0}, \cdots, X_{m}, \cdots$ are the pairwise isomorphic Hilbert spaces over $\boldsymbol{R}$, also $X_{\boldsymbol{R}}=X_{0} \oplus X_{1} \oplus \cdots \oplus X_{m} \oplus \cdots$. The scalar product

$\langle x ; y\rangle$ in $X$ then can be written in the form

$$
\text { 1) }\langle x ; y\rangle=\sum_{k, n}\left\langle x_{k} ; y_{n}\right\rangle \tilde{i}_{k} i_{n},
$$

where $\left\langle x_{k} ; y_{n}\right\rangle \in \boldsymbol{R}$ due to $\S 2.8$ above. Then the scalar product $\langle x ; y\rangle$ in $X$ induces the scalar product.

$$
\text { 2) }\langle x ; y\rangle_{\boldsymbol{R}}:=\sum_{k}\left\langle x_{k} ; y_{k}\right\rangle \text {. }
$$

in $X_{R}$. Therefore, the orthogonality of $x$ to the subspace $Y$ relative to $\langle x ; y\rangle$ is equivalent to $\left\langle x_{k} ; y_{n}\right\rangle=0$ for every $y \in Y$ and each $k, n$, that implies the orthogonality of $x$ to the subspace $Y_{R}$ relative to $\langle x ; y\rangle_{R}=(x, y)$. Due to Lemma 31 from $y \in \boldsymbol{Y}$ it follows, that $y_{n} i_{n} \in Y$ for each $n=0,1, \cdots, m, \cdots$. If $y_{n} \in Y_{n}$, then $y_{n} i_{k}^{*} i_{n} \in Y_{l} i_{l}$, where either $i_{l}=i_{k}^{*} i_{n}$ or $i_{l}=-i_{k}^{*} i_{n}$. Moreover, by the definition $Y$ is the two-sided module over the CayleyDickson algebra $\mathcal{A}_{v}$. Then from $\left\langle x ; y_{k}\right\rangle_{R}=0$ for each $y \in Y$ and $k=0,1, \cdots$ due to Formula $2.8(S P)$ it follows, that $\left\langle x_{k} ; y_{n}\right\rangle=0$ for each $k, n$, since $Y$ is the $\mathcal{A}_{v}$-vector space, consequently, $\langle x ; y\rangle=0$ for each $y \in Y$.

Then by the theorem about transfinite induction [14] in $X$, the orthogonal basis over $\mathcal{A}_{v}$ exists, in which every vector can be presented in the form of the converging series of left (or right) $\mathcal{A}_{v}$-vector combinations of basic vectors. The real Hilbert space $X_{0}$ is isomorphic with $l_{2}(\alpha, \boldsymbol{R})$, consequently, $X=X_{0} \oplus X_{1} i_{1} \oplus \cdots \oplus X_{m} i_{m} \oplus \cdots$ is isomorphic with $l_{2}\left(\alpha, \mathcal{A}_{v}\right)$. The Cayley-Dickson algebra $\mathcal{A}_{v}$ is normed, while the real field $\boldsymbol{R}$ is separable, hence $d\left(\mathcal{A}_{v}\right)=\aleph_{0} 2^{\operatorname{card}(v)}$. The space $X$ is normed, consequently, the base of neighborhoods of $x$ is countable for each $x \in X$, hence for the topological density it is accomplished the equality $d(X)=\operatorname{card}(\alpha) \aleph_{0} 2^{\operatorname{card}(v)}$. Particularly, for a finite dimensional algebra $\mathcal{A}_{v}$, i.e. $2 \leq v<\infty$, and $\operatorname{card}(\alpha) \geq \aleph_{0}$ one gets $d(X)=\operatorname{card}(\alpha)$.

31) Lemma. For each quasi-linear operator $T$ in a Hilbert space $X$ over the Cayley-Dickson algebra $\mathcal{A}_{v}$ an adjoint operator $T^{*}$ in $X$ relative to the $\mathcal{A}_{v}$-scalar product induces an adjoint operator $T_{R}^{*}$ in $X_{R}$ relative to the $\boldsymbol{R}$-valued scalar product in $X_{R}$.

Proof. Let $\mathcal{D}(T)$ be a domain of the definition of an operator $T$, which is dense in $X$. Due to Formulas 30 $(i, i i)$ and the existence of the $\boldsymbol{R}$-linear automorphisms $z \mapsto z i_{k}$ in $\mathcal{A}_{v}$ as the $\boldsymbol{R}$-linear space for each $k=0,1, \cdots, m, \cdots$, the continuity of scalar products $\langle T x ; y\rangle$ implies that of $\langle T x ; y\rangle_{R}$ by $x \in \mathcal{D}(T)$. For $v<\infty$ these continuities are equivalent. Therefore, due 
to Lemma 29 the family of all $y \in X$, for which $\langle T x ; y\rangle$ is continuous by $x \in \mathcal{D}(T)$ forms an $\mathcal{A}_{v}$-vector subspace in $X$ and this provides a domain of the definition $\mathcal{D}\left(T^{*}\right)$ of the operator $T^{*}$ everywhere dense in $X$. Then the adjoint operator $T^{*}$ is defined by the equality $\langle T x ; y\rangle=:\left\langle x ; T^{*} y\right\rangle$, also $T_{R}^{*}$ is given by the way of $\langle T x ; y\rangle_{\boldsymbol{R}}=\left\langle x ; T_{R}^{*} y\right\rangle_{\boldsymbol{R}}$, where $x \in \mathcal{D}(T)$, also $y \in \mathcal{D}\left(T^{*}\right)$. Due to Formula $30(\mathrm{i}, \mathrm{ii})$ one deduces $\left\langle x_{k} ;\left(T^{*} y\right)_{k}\right\rangle=\left\langle x_{k} ;\left(T^{*} y\right)_{k}\right\rangle_{\boldsymbol{R}}$ for each $x \in \mathcal{D}(T)$, $y \in \mathcal{D}\left(T^{*}\right)$ and $k=0,1, \cdots, m, \cdots$. In view of Propositions 2.22.18 and 2.22.20 [18] and Lemma 29 above $\mathcal{D}(T)$ and $\mathcal{D}\left(T^{*}\right)$ are $\mathcal{A}_{v}$-vector spaces, then the family of $\boldsymbol{R}$-linear automorphisms of the CayleyDickson algebra $\mathcal{A}_{\text {, }}$ as the $\boldsymbol{R}$-linear space given above lead to the conclusion that $T^{*}$ on $\mathcal{D}\left(T^{*}\right)$ induces $T_{\boldsymbol{R}}^{*}$ in $X_{R}$.

32) Definition. A bounded quasi-linear operator $P$ in a Hilbert space $X$ over the Cayley-Dickson algebra $\mathcal{A}_{v}$ is called a partial $\boldsymbol{R}$ - (or $\mathcal{A}_{v}$-) isometry, if there exists a closed $\boldsymbol{R}$ - (or $\mathcal{A}_{v}$-) vector subspace $Y$ such that $\|P x\|=\|x\|$ for each $x \in Y$ and $P\left(Y_{\boldsymbol{R}}^{\perp}\right)=\{0\}$ (or $P\left(Y^{\perp}\right)=\{0\}$ respectively), where

$$
\begin{aligned}
& Y^{\perp}:=\{z \in X:\langle z ; y\rangle=0, \forall y \in Y\} \\
& Y_{R}^{\perp}:=\left\{z \in X_{\boldsymbol{R}}:\langle z ; y\rangle_{\boldsymbol{R}}=0, \forall y \in Y\right\} .
\end{aligned}
$$

33) Definition. An operator $T: X \rightarrow Y$ with an $\mathcal{A}_{v}$ vector domain $\mathcal{D}(T)$ in a Hilbert space $X$ over the Cayley-Dickson algebra $\mathcal{A}_{v}$ is called densely defined, if $\mathcal{D}(T)$ is (every)where dense in $X$, where $Y$ is a Hilbert space over $\mathcal{A}_{v}$.

An operator $Q$ extends $T$ (or $Q$ is an extension of $T$ ) if $\mathcal{D}(Q) \subset \mathcal{D}(T)$ and $Q x=T x$ for each $x \in \mathcal{D}(Q)$. This situation is denoted by $Q \subseteq T$.

Denote by $\Gamma(T):=\{(x, T x): x \in \mathcal{D}(T)\}$ a graph of $T$. If $c l[\Gamma(T)]$ is the graph of a quasi-linear operator $\bar{T}$, then one says that $T$ is pre-closed (or closable) and refers to $\bar{T}$ as the closure of $T$.

34) Theorem. Let $T$ be a densely defined quasi-linear operator $T: X \rightarrow Y$ in a Hilbert space $X$ over the Cayley-Dickson algebra $\mathcal{A}_{v}$, where $Y$ is a Hilbert space over $\mathcal{A}_{v}, 0 \leq v$. Suppose that $T$ is either left or right $\mathcal{A}_{v}$ linear. Then

1) If T is pre-closed, then $(\bar{T})^{*}=T^{*}$;

2) $T$ is pre-closed if and only if $\mathcal{D}\left(T^{*}\right)$ is dense in $Y$;

3) If $T$ is pre-closed, then $T^{* *}=\bar{T}$;

4) If $T$ is closed, then the operator $\left(I+T^{*} T\right)$ is oneto-one with range $X$ and positive inverse $\left(I+T^{*} T\right)^{-1}$ of norm not exceeding 1 ;

5) If $T$ is closed, then the operator $T^{*} T$ is self-adjoint and positive;

6) If $T$ is either left or right $\mathcal{A}_{v}$-linear on $\mathcal{D}(T)$, then
$T^{*}$ is either left or right $\mathcal{A}_{v}$-linear on $\mathcal{D}\left(T^{*}\right)$ respectively.

Proof. If an operator $T$ is either left or right $\mathcal{A}_{v}$-linear, then $\Gamma(T)$ is an $\mathcal{A}_{v}$-vector space due to Lemma 29. Therefore, consider the case of $\Gamma(T)$ being an $\mathcal{A}_{v}$ vector space.

6) Let $T$ be right $\mathcal{A}_{v}$-linear $x_{0} \in \mathcal{D}(T)_{0}$ and $y_{0} \in \mathcal{D}\left(T^{*}\right)_{0}, \quad z \in \mathcal{A}_{v}$, then

$$
\begin{aligned}
\left\langle T\left(x_{0} a\right) ; y_{0}\right\rangle & =\left\langle\left(T x_{0}\right) a ; y_{0}\right\rangle=\left\langle T x_{0} ; y_{0}\right\rangle a^{*}=\left\langle x_{0} ; T^{*} y_{0}\right\rangle a^{*} \\
& =\left\langle x_{0} ;\left(T^{*} y_{0}\right) a^{*}\right\rangle=\left\langle T x_{0}, y_{0} a^{*}\right\rangle \\
& =\left\langle x_{0} ; T\left(y_{0} a^{*}\right)\right\rangle
\end{aligned}
$$

in accordance with Formula $2.8(S P)$, consequently, $T\left(y_{0} b\right)=\left(T y_{0}\right) b$ for each $b \in \mathcal{A}_{v}$, since

$X_{0} \ni x_{0} \mapsto x_{0} i_{j} \in X_{j} i_{j}$ induces the $\boldsymbol{R}$-linear isomorphism of $X_{0}$ onto $X_{j}, \quad\left(y_{0} b\right) i_{j}=y_{0}\left(b i_{j}\right)$ for each $j=0,1,2, \cdots$. Analogously Statement (6) of this theorem is verified in the case of a left $\mathcal{A}_{v}$-linear operator $T$.

1) If $Q$ is densely defined and $T$ is an extension of $Q$, then $\langle Q x ; y\rangle=\langle T x ; y\rangle=\left\langle x ; T^{*} y\right\rangle$ for each $y \in \mathcal{D}\left(T^{*}\right)$ and $x \in \mathcal{D}(Q)$, consequently, $y \in \mathcal{D}\left(Q^{*}\right)$ and

$Q^{*} y=T^{*} y$, hence $Q^{*}$ is an extension of $T^{*}$. From $T \subseteq \bar{T}$ it follows that $(\bar{T})^{*} \subseteq T^{*}$. For an arbitrary vector $x \in X$ take a sequence $\left\{{ }_{n} x: n\right\}$ in $\mathcal{D}(T)$ converging to $x$ such that $\left\{T_{n} x\right\}$ converges to $\bar{T} x$. Therefore,

$$
\langle\bar{T} x ; y\rangle=\lim _{n}\left\langle T_{n} x ; y\right\rangle=\lim _{n}\left\langle{ }_{n} x ; T^{*} y\right\rangle=\left\langle x ; T^{*} y\right\rangle
$$

for each $y \in \mathcal{D}\left(T^{*}\right)$, consequently, $y \in \mathcal{D}\left((\bar{T})^{*}\right)$ and $(\bar{T})^{*} y=T^{*} y$. Thus $(\bar{T})^{*}=T^{*}$.

2) If $\Gamma(T)$ is closed in $X \times Y$, then it is the Hilbert space over $\mathcal{A}_{v}$ so that $\Gamma(T) \subset X \times Y$. Consider the mapping $P: \Gamma(T) \ni(x, y) \mapsto x \in Y$, consequently, $P$ is bounded and quasi-linear. Thus $P$ has a bounded adjoint $P^{*}$ mapping $Y$ into $\Gamma(T)$. This operator $P$ has null space $\operatorname{ker}(P)=\{0\}$, since $(0, y) \in \Gamma(T)$ only when $y$ $=0$. In view of Theorem 2.30 [18] the range of $P^{*}$ is dense in $\Gamma(T)$ as the $\mathcal{A}_{v}$-vector space. Thus $\Gamma(\bar{T})$ contains a dense $\mathcal{A}_{v}$-vector subspace the range of $P^{*}$ consisting of all pairs $(x, \overline{T x})$ with

$\bar{T} x \in \mathcal{D}\left(T^{*}\right)=\mathcal{D}\left(\bar{T}^{*}\right)$. If a vector $y$ is orthogonal to $R(\bar{T})$, then $0=\langle\bar{T} x ; y\rangle=\langle T x ; y\rangle$ for each $x \in \mathcal{D}(T)$, hence $y \in \mathcal{D}\left(T^{*}\right)$ and it is annihilated by $T^{*}$. Thus $\mathcal{D}\left(T^{*}\right)$ contains a dense subset of the $\mathcal{A}_{v}$-vector range of $\bar{T}$ as well as the orthogonal complement of this range due to Lemma 31. The formula of the scalar product $2.8(S P)$ on $X$ and Cauchy-Schwartz' inequality implies that there is the $\mathcal{A}_{v}$-vector subspace $\mathcal{D}\left(T^{*}\right)$ in $Y$, since $\mathcal{D}(T)$ is the $\mathcal{A}_{v}$-vector subspace. Since $\mathcal{D}\left(T^{*}\right)$ is an $\mathcal{A}_{v}$-vector subspace, it is dense in $Y$.

Suppose now that $\mathcal{D}(T)$ is dense in $Y$ and $\left\{{ }_{n} x: n\right\}$ is a sequence in $\mathcal{D}(T)$ converging to zero so that 
$\left\{T_{n} x: n\right\}$ converges to $w$. Then if $y \in \mathcal{D}\left(T^{*}\right)$ we get $\left\langle T_{n} x ; y\right\rangle=\left\langle{ }_{n} x ; T^{*} y\right\rangle$, hence $\left\langle{ }_{n} x ; T^{*} y\right\rangle$ converges to 0 and $\langle w ; y\rangle$ simultaneously. Therefore, the operator $T$ is pre-closed, since $\mathcal{D}\left(T^{*}\right)$ is dense in $Y$ and hence $w=0$.

3) At first we demonstrate that if $T$ is densely defined, then $T^{*}$ is a closed operator. Take arbitrary vectors $y, z \in \mathcal{D}\left(T^{*}\right)$ and $x \in \mathcal{D}(T)$. Let $T$ be left $\mathcal{A}_{v}$-linear, so we get

$$
\begin{aligned}
& \left\langle x_{j} ; T^{*}\left(a \sum_{k} y_{k} i_{k}\right)+T^{*} z\right\rangle \\
& =\left\langle T x_{j} ; a\left(\sum_{k} y_{k} i_{k}\right)+z\right\rangle=\left\langle T x_{j} ; a\left(\sum_{k} y_{k} i_{k}\right)\right\rangle+\left\langle T x_{j} ; z\right\rangle \\
& =\left\langle T\left(x_{j} a^{*}\right) ; \sum_{k} y_{k} i_{k}\right\rangle+\left\langle T x_{j} ; z\right\rangle \\
& =\left\langle x_{j} a^{*} ; T^{*}\left(\sum_{k} y_{k} i_{k}\right)\right\rangle+\left\langle x_{j} ; T^{*} z\right\rangle \\
& =\left\langle x_{j} ; a T^{*}\left(\sum_{k} y_{k} i_{k}\right)\right\rangle+\left\langle x_{j} ; T^{*} z\right\rangle
\end{aligned}
$$

for each $a \in \boldsymbol{R} i_{l}$ and $y=\sum_{k} y_{k} i_{k} \in \mathcal{D}\left(T^{*}\right) \subset Y$ and $z \in \mathcal{D}\left(T^{*}\right)$, since the subalgebra $\operatorname{alg}_{\boldsymbol{R}}\left(i_{k}, i_{l}\right)$ is associative for each $k, l$, consequently, $a y+z \in \mathcal{D}\left(T^{*}\right)$ and $T^{*}\left(a y_{k}+z\right)=a T^{*} y_{k}+T^{*} z$. Thus $\mathcal{D}\left(T^{*}\right)$ is an $\mathcal{A}_{v}$-vector space by Lemmas 30 and 31 and $T^{*}$ is a left $\mathcal{A}_{v}$-linear operator, since each Cayley-Dickson number $b$ has the decomposition $b=\sum_{l} b_{l} i_{l}$ with $b_{l} \in \boldsymbol{R}$ for every

l. Analogously it can be demonstrated in the right $\mathcal{A}_{v}$ linear case (see also (6) above). If $\left\{{ }_{n} z: n\right\}$ is a sequence in $\mathcal{D}\left(T^{*}\right)$ converging to $z$ so that $\left\{T^{*}{ }_{n} z: n\right\}$ converges to $w$, then $\left\langle y ; T^{*}{ }_{n} z\right\rangle=\left\langle T y ;{ }_{n} z\right\rangle$, hence the sequence $\left\{\left\langle y ; T^{*}{ }_{n} z\right\rangle: n\right\}$ converges to $\langle T y ; z\rangle$ and inevitably this gives $\langle T y ; z\rangle=\langle y ; w\rangle$ for each $y \in \mathcal{D}(T)$. Thus $z \in \mathcal{D}\left(T^{*}\right)$ and $T^{*} z=w$. This means that the operator $T^{*}$ is closed.

Suppose that conditions of (3) are accomplished. The domain of definition $\mathcal{D}\left(T^{*}\right)$ is dense in $X$ in accordance with (2), when $T$ is pre-closed, so that $T^{*}$ has an adjoint operator $T^{* *}$. Then $\left\langle T^{*} y ; x\right\rangle=\langle y ; T x\rangle$ for each $x \in \mathcal{D}(T)$ and $y \in \mathcal{D}\left(T^{*}\right)$, consequently, $x \in \mathcal{D}\left(T^{* *}\right)$ and $T^{* *} x=T x$. Therefore, the operator $T^{* *}$ is closed, consequently, $T \subseteq \bar{T} \subseteq T^{* *}$. From Section 1 of this proof, it follows that $T^{* * *} \subseteq \bar{T}^{*}=T^{*}$. On the other hand, we have $T^{*} \subseteq\left(T^{*}\right)^{* *}$, since the operator $T^{*}$ is closed. Thus $T^{*}=T^{* * *}$.

We have that $\bar{T} \subseteq T^{* *}$ and $\Gamma(\bar{T}) \subseteq \Gamma\left(T^{* *}\right)$. This implies that $\langle x ; y\rangle+\left\langle T^{* *} x ; \bar{T} y\right\rangle=0$ for each $y \in \mathcal{D}(\bar{T})$ when $\left(x, T^{* *} x\right) \in \Gamma\left(T^{* *}\right)$ is orthogonal to $\Gamma(\bar{T})$. Par- ticularly it holds for $\overline{T y} \in \mathcal{D}\left(T^{*}\right)$ and implies $\left\langle x ;\left(T^{*} \bar{T}+I y\right)\right\rangle=0$.

From (2) of this proof we get that $\left(T^{*} \bar{T}+I\right) X=X$. Indeed, if $z \in X$ and $P^{*} z=(w, q)$, then $T w=q$ and

$$
\langle z ; y\rangle=\left\langle P^{*} z ;(y, T y)\right\rangle=\langle w ; y\rangle+\langle q ; T y\rangle
$$

for each $y \in \mathcal{D}(T)$, consequently, $\langle T y ; q\rangle=\langle y ; z-w\rangle$ and $q \in \mathcal{D}\left(T^{*}\right)$. Then $T^{*} q=T^{*} T w=z-w$ and $\left(T^{*} T+I\right) w=z$. Thus $x=0$ and $\Gamma\left(T^{* *}\right)=\Gamma(\bar{T})$ and $T^{* *}=\bar{T}$.

4) If $x \in \mathcal{D}\left(T^{*} T+I\right)$, then

$$
\begin{aligned}
\|x\|^{2} & \leq\langle x ; x\rangle+\langle T x ; T x\rangle=\left\langle\left(T^{*} T+I\right) x ; x\right\rangle \\
& \leq\left\|\left(T^{*} T+I\right) x\right\|\|x\| .
\end{aligned}
$$

Hence $\operatorname{ker}\left(T^{*} T+I\right):=\left(T^{*} T+I\right)^{-1}(0)=\{0\}$, that is, this operator $\left(T^{*} T+I\right)$ is one-to-one and has a bounded inverse of norm not exceeding one. Each $z \in X$ has the form $\left(T^{*} T+I\right) x=z$, consequently, the inverse operator $H:=\left(T^{*} T+I\right)^{-1}$ is positive, since $\langle z ; H z\rangle=\left\langle\left(T^{*} T+I\right) x ; x\right\rangle$.

5) From 4) we have that $\mathcal{D}\left(T^{*} T\right)$ is dense in $X$. For each $x \in \mathcal{D}\left(T^{*} T\right)$ we get

$$
\left\langle\left(T^{*} T+I\right) x ; y\right\rangle=\left\langle T^{*} T x ; y\right\rangle+\langle x ; y\rangle,
$$

consequently, $\left(T^{*} T\right)^{*}$ and $\left(T^{*} T+I\right)^{*}$ have the same domain and $\left(T^{*} T\right)^{*}+I=\left(T^{*} T+I\right)^{*}$. With $y \in \mathcal{D}\left(T^{*} T\right)$ one has the equality $\left\langle T^{*} T x ; y\right\rangle=\left\langle x ; T^{*} T y\right\rangle$ such that $T^{*} T \subseteq\left(T^{*} T\right)^{*}$ and $\left(T^{*} T+I\right) \subseteq\left(T^{*} T+I\right)^{*}$, consequently, $\left(T^{*} T+I\right)^{*} X=X$. If a vector $y$ is such that $\left(T^{*} T+I\right)^{*} y=0$, then

$$
\left\langle\left(T^{*} T+I\right)^{*} y ; x\right\rangle=\left\langle y ;\left(T^{*} T+I\right) x\right\rangle=0
$$

for each $x \in \mathcal{D}\left(T^{*} T\right)$, consequently, $y=0$, since

$$
R\left(T^{*} T+I\right):=\left(T^{*} T+I\right) X=X .
$$

Thus the operator $\left(T^{*} T+I\right)^{*}$ is bijective and extends the operator $\left(T^{*} T+I\right)$ and these two operators have the same range. Therefore, one infers that

$T^{*} T+I=\left(T^{*} T+I\right)^{*}=\left(T^{*} T\right)^{*}+I$ so that $T^{*} T=\left(T^{*} T\right)^{*}$. Then the inequality $\left\langle T^{*} T x ; x\right\rangle=\langle T x ; T x\rangle \geq 0$ for each $x \in \mathcal{D}\left(T^{*} T\right)$ shows that the operator $T^{*} T$ is positive.

35) Theorem. If $T$ is a closed quasi-linear $\mathcal{A}_{r}$-metainvariant operator in a Hilbert space X over the CayleyDickson algebra $\mathcal{A}_{v}$, where $2 \leq v, 1 \leq r \leq \min (3, v)$, then $T=P A$, where $P$ is a partial $\mathbf{R}$-isometry on $X_{\boldsymbol{R}}$ with 
the initial domain $\operatorname{cl}\left(\mathcal{R}\left(T^{*}\right)\right)$, also $A$ is a self-adjoint quasi-linear operator such that $\operatorname{cl}(\mathcal{R}(A))=\operatorname{cl}\left(\mathcal{R}\left(T^{*}\right)\right)$, where $\mathcal{R}(T)$ denotes a range of $T$. If additionally $T$ is either left or right $\mathcal{A}_{v}$-linear, then there are either left or right $\mathcal{A}_{v}$-linear isometry $P$ and either left or right $\mathcal{A}_{v}$-linear operator A respectively such that $T=P A$.

Proof. Due to the spectral theorem 21, a self-adjoint quasi-linear $\mathcal{A}_{r}$-meta-invariant operator $T$ is positive if and only if its spectrum is contained in $\sigma(T) \subset[0, \infty)$. In the Cayley-Dickson algebra $\mathcal{A}_{v}$ each polynomial has a root, i.e. zero, (see Theorem 3.17 [16] or §2.2.4 [13]). Therefore, if $H$ is a positive self-adjoint quasi-linear operator, then there exists a unique positive quasi-linear $\mathcal{A}_{r}$-meta-invariant operator $A$, such that $A^{2}=H$,

$$
A=\int_{0}^{\infty} \sqrt{b} \hat{\boldsymbol{E}}(\mathrm{d} b)
$$

With $f(b)=\sqrt{b} \geq 0$ for $b \geq 0$ and a domain of its definition $\mathcal{D}(A)$ is given by Theorem 21. Therefore, generally there exists a positive square root $A$ of the operator $T^{*} T$. The space $\operatorname{cl}(\mathcal{R}(A))$ is $\boldsymbol{R}$-linear, where $\mathcal{R}(A):=A X$.

If the operator $A$ is in addition either left or right $\mathcal{A}_{v}$ linear, then $\operatorname{cl}(\mathcal{R}(A))$ is the $\mathcal{A}_{v}$-vector subspace due to Lemma 29. In view of Lemma 30, there exists the perpendicular projection $\hat{\boldsymbol{E}}([0, \infty))$ from $X$ on $\operatorname{cl}(\mathcal{R}(A))$, moreover, $\hat{\boldsymbol{E}}$ is right or left $\mathcal{A}_{v}$-linear, if $\operatorname{cl}(\mathcal{R}(A))$ is the $\mathcal{A}_{v}$-vector subspace.

Then $Q$ is a correctly defined isometry with the domain of definition $\mathcal{R}(B)$, where $B$ is the restriction of $A$ on $\mathcal{D}\left(T^{*} T\right), \quad B=\left.A\right|_{\mathcal{D}\left(T^{*} T\right)}$, where $\mathcal{D}\left(T^{*} T\right)$ is considered as the $\boldsymbol{R}$-linear space embedded into $X$ in general, but for either left or right $\mathcal{A}_{v}$-linear operator $T$ it is considered as the $\mathcal{A}_{v}$-vector space, analogously for domains and ranges of the considered here other operators, that is clear from the context. In view of Theorem $34 \Gamma(B)$ is dense in $\Gamma(A)$, since $T^{*} T=A^{*} A$. Therefore, if $(x, y) \in \Gamma(A)$, then there exists a sequence $\left({ }_{n} x,{ }_{n} y\right)$ in $\Gamma(B)$ converging to $(x, y)$, consequently, $\lim _{n} y=y$ and the vector space $\mathcal{R}(B):=B X$ is dense in

$\mathcal{R}(A):=A X$. Let $P^{1}$ be an isometric extension of the operator $Q$ on $c l[\mathcal{R}(A)]$ and $F$ be a perpendicular projection in $X$ on $c l[\mathcal{R}(A)]$. If put $P=P^{1} F$, then $P$ is a partial isometry with an initial domain $\operatorname{cl}[\mathcal{R}(A)]$. Moreover, $P A x=T x$ for every $x \in \mathcal{D}\left(T^{*} T\right)$.

Let $\left\{{ }_{n} y: n\right\}$ be a sequence in $\mathcal{D}(A)$ such that $\lim _{n n} y=y$ and $\lim _{n} P A_{n} y=z$. Then there exists the limit $\lim _{n} A_{n} y=w$, since $P$ is the isometric $\boldsymbol{R}$-linear mapping. Then $y \in \mathcal{D}(A)$ and $A y=w$, since the operator $A$ is closed. Thus $y \in \mathcal{D}(P A)$ and $P A y=z$, consequently, the operator $P A$ is closed.

It is necessary to verify that $T=P A$. Let $K$ be a restriction of $T$ on $\mathcal{D}\left(T^{*} T\right)$. Then by Theorem $34 \Gamma(K)$ is dense in $\Gamma(T)$. If $x \in \mathcal{D}(T)$, there exists a sequence $\left\{\left({ }_{n} x, A_{n} x\right): n\right\}$ in $\Gamma(K)$ such that $\lim _{n}\left({ }_{n} x, A_{n} x\right)=(x, A x)$. From $P A_{n} x=T_{n} x$ and closeness of $P A$ it follows that

$$
P A x=\lim _{n} P A_{n} x=\lim _{n} T_{n} x=T x,
$$

consequently, $P A \subseteq T$.

We have that $\Gamma(B)$ is dense in $\Gamma(A)$. Therefore, for each $x \in \mathcal{D}(A)$ a sequence $\left\{\left({ }_{n} x, A_{n} x\right): n\right\} \subset \Gamma(B)$ exists so that $\lim _{n}\left({ }_{n} x, A_{n} x\right)=(x, A x)$. Then we deduce that $P A x=\lim _{n} P A_{n} x=\lim _{n} T_{n} x=T x$, since

$P A_{n} x=T_{n} x$ and the operator $T$ is closed. Thus $P A \subseteq T$, consequently, together with the opposite inclusion demonstrated above, it implies that $P A=T$.

In view of Theorem 34 one gets

$$
c l[\mathcal{R}(A)]=\operatorname{cl}\left[\mathcal{R}\left(T^{*}\right)\right],
$$

since $A^{*} x=A x=0$ if and only if $T^{* *}=T x=P A x=0$.

We next demonstrate that this decomposition $T=P A$ into the product of operators is unique. In accordance with Theorem $34 A P^{*}=T^{*}$, consequently, $T^{*} T=A P^{*} P A$. On the other hand, $P^{*} P$ is the projector on $\operatorname{cl}[\mathcal{R}(A)]$, hence $T^{*} T=A^{2}$. A uniqueness of $A$ follows from Theorem 21. Since the mapping $A$ is unique, the operator $P$ is defined on $\mathcal{R}(A)$ by the equation $P(A x)=T x$ in the unique manner. A continuous extension of $P$ from $\mathcal{R}(A)$ on $\operatorname{cl}[\mathcal{R}(A)]$ is unique. The restriction of operator $P$ on the orthogonal complement $\operatorname{cl}[\mathcal{R}(A)]^{\perp}$ is zero. Thus the operator $P$ is uniquely defined by the operator $T$.

At the same time $A$ is either right or left $\mathcal{A}_{v}$-linear, if $T$ is either right or left $\mathcal{A}_{v}$-linear, since so is $\hat{\boldsymbol{E}}$ for such $T$ and also $T^{*}$ is either right or left $\mathcal{A}_{v}$-linear due to Theorem 34(6). Since $T$ and $A$ are either right or left $\mathcal{A}_{v}$-linear, then $P$ also should be such.

The presented above results of this paper and from works $[16,20-30]$ can be used for further developments of the operator theory over the Cayley-Dickson algebras including that of PDO.

\section{REFERENCES}

[1] A. Connes, "Non-Commutative Geometry," Academic Press, San Diego, 1994.

[2] F. van Oystaeyen, "Algebraic Geometry for Associative Algebras," Lecture Notes in Pure and Applied Mathemematics, Vol. 232, Marcel Dekker, New York, 2000.

[3] G. Emch, "Mèchanique Quantique Quaternionienne et Relativitè Restreinte,” Helvetica Physica Acta, Vol. 36, 1963, pp. 739-788.

[4] S. V. Ludkovsky, “Generalized Geometric Loop Groups of Complex Manifolds, Gaussian Quasi-Invariant Measures on Them and Their Representations," Journal of Mathematical Sciences, Vol. 122, No. 1, 2004, pp. 29843010. doi:10.1023/B:JOTH.0000029575.92350.db 
[5] S. V. Ludkovsky, "Poisson Measures for Topological Groups and Their Representations,” Southeast Asian Bulletin of Mathematics, Vol. 25, No. 4, 2002, pp. 653-680. doi:10.1007/s100120200010

[6] S. V. Ludkovsky, "Wrap Groups of Connected Fiber Bundles: Their Structure and Cohomologies,” International Journal of Mathematics, Game Theory and Algebra, Vol. 19, No. 1-2, 2009, pp. 53-128.

[7] S. V. Ludkovsky, "Affine and Wrap Algebras over Octonions,” Journal of Mathematical Sciences, Vol. 167, No. 6, 2010, pp. 767-809. doi:10.1007/s10958-010-9960-7

[8] S. V. Ludkovsky, "Wrap Groups of Fiber Bundles over Quaternions and Octonions," CUBO A Mathematical Journal, Vol. 11, No. 1, 2009, pp. 101-121.

[9] H. B. Lawson and M.-L. Michelson, "Spin Geometry," Princeton University Press, Princeton, 1989.

[10] S. V. Ludkovsky, "Functions of Several Cayley-Dickson Variables and Manifolds over Them," Journal of Mathematical Sciences, Vol. 141, No. 3, 2007, pp. 1299-1330. doi:10.1007/s10958-007-0043-3

[11] A. G. Kurosh, "Lectures on General Algebra,” Nauka, Moscow, 1973.

[12] S. V. Ludkovsky, "Algebras of Operators in Banach Spaces over the Quaternion Skew Field and the Octonion Algebra,” Journal of Mathematical Sciences, Vol. 144, No. 4, 2008, pp. 4301-4366. doi:10.1007/s10958-007-0273-4

[13] S. V. Ludkovsky, “Analysis over Cayley-Dickson Numbers and Its Applications,” LAP Lambert Academic Publishing, Saarbrücken, 2010.

[14] R. Engelking, “General Topology,” Heldermann, Berlin, 1989.

[15] L. Narici and E. Beckenstein, "Topological Vector Spaces,” Marcel-Dekker, New York, 1985.

[16] S. V. Ludkovsky, "Differentiable Functions of CayleyDickson Numbers and Line Integration,” Journal of Mathematical Sciences, Vol. 141, No. 3, 2007, pp. 12311298. doi:10.1007/s10958-007-0042-4

[17] A. Kolmogorov and S. Fomine, "Éléments de la Théorie des Fonctions et de L'analyse Fonctionnelle,” Ellipses, Paris, 1994.

[18] S. V. Ludkovsky, "Algebras of Operators in Banach Spaces over the Quaternion Skew Field and the Octonion Algebra,” Journal of Mathematical Sciences, Vol. 144, No. 4, 2008, pp. 4301-4366. doi:10.1007/s10958-007-0273-4
[19] N. Dunford and J. C. Schwartz, "Linear Operators,” Wiley and Sons, Inc., New York, 1966.

[20] L. E. Dickson, “The Collected Mathematical Papers,” Vol. 1-5, Chelsea Publishing Co., New York, 1975.

[21] K. Gürlebeck and W. Sprössig, "Quaternionic and Clifford Calculus for Physicists and Engineers,” John Wiley and Sons, Chichester, 1997.

[22] K. Gürlebeck and W. Sprössig, "Quaternionic Analysis and Elliptic Boundary Value Problem,” Birkhäuser, Basel, 1990. doi:10.1007/978-3-0348-7295-9

[23] R. V. Kadison and J. R. Ringrose, "Fundamentals of the Theory of Operator Algebras,” Academic Press, New York, 1983.

[24] I. L. Kantor and A. S. Solodovnikov, "Hypercomplex Numbers,” Springer-Verlag, Berlin, 1989. doi:10.1007/978-1-4612-3650-4

[25] S. V. Ludkovsky and F. van Oystaeyen, "Differentiable Functions of Quaternion Variables,” Bulletin des Sciences Mathematiques (Paris) Series 2, Vol. 127, 2003, pp. 755796.

[26] S. V. Ludkovsky, "Stochastic Processes on Geometric Loop Groups, Diffeomorphism Groups of Connected Manifolds, Associated Unitary Representations,” Journal of Mathematical Sciences, Vol. 141, No. 3, 2007, pp. 13311384. doi:10.1007/s10958-007-0044-2

[27] S. V. Ludkovsky, "Differential Equations over Octonions," Advances in Applied Clifford Algebras, Vol. 21, No. 4, 2011, pp. 773-797. doi:10.1007/s00006-011-0286-4

[28] S. V. Ludkovsky and W. Sproessig, "Ordered Representations of Normal and Super-Differential Operators in Quaternion and Octonion Hilbert Spaces," Advances in Applied Clifford Algebras, Vol. 20, No. 2, 2010, pp. 321-342. doi:10.1007/s00006-009-0180-5

[29] S. V. Ludkovsky and W. Sprössig, "Spectral Theory of Super-Differential Operators of Quaternion and Octonion Variables," Advances in Applied Clifford Algebras, Vol. 21, No. 1, 2011, pp. 165-191. doi:10.1007/s00006-010-0238-4

[30] S. V. Ludkovsky and W. Sprössig, "Spectral Representations of Operators in Hilbert Spaces over Quaternions and Octonions," Complex Variables and Elliptic Equations, Vol. 57, No. 2, 2012, pp. 1301-1324. doi:10.1080/17476933.2010.538845 